\title{
LACUNARY FOURIER SERIES FOR COMPACT QUANTUM GROUPS
}

\author{
SIMENG WANG
}

\begin{abstract}
This paper is devoted to the study of Sidon sets, $\Lambda(p)$-sets and some related notions for compact quantum groups. We establish several different characterizations of Sidon sets, and in particular prove that any Sidon set in a discrete group is a strong Sidon set in the sense of Picardello. We give several relations between Sidon sets, $\Lambda(p)$-sets and lacunarities for $L^{p}$ Fourier multipliers, generalizing a previous work by Blendek and Michaliček. We also prove the existence of $\Lambda(p)$-sets for orthogonal systems in noncommutative $L^{p}$-spaces, and deduce the corresponding properties for compact quantum groups. Central Sidon sets are also discussed, and it turns out that the compact quantum groups with the same fusion rules and the same dimension functions have identical central Sidon sets. Several examples are also included.
\end{abstract}

\section{INTRODUCTION}

The study of lacunarity and particularly of Sidon sets is a major and fascinating subject of harmonic analysis. Historically, the notion of Sidon sets originated from discussions of special lacunary series on the circle $\mathbb{T}$. This has been generalized later by replacing $\mathbb{T}$ with an arbitrary compact abelian group $G$. Recall that for a compact abelian group $G$, a Sidon set $\mathbf{E}$ for $G$ is a subset of the dual discrete group $\Gamma=\hat{G}$ such that any continuous function on $G$ with Fourier transform supported on $\mathbf{E}$ has absolutely convergent Fourier series. The theory of Sidon sets and lacunarity for compact abelian groups has been remarkably developed in the past several decades, and in a series of works of Rudin, Drury, Rider, Marcus, Pisier and others (see [Dru70, Rid75, Rud60, MP81, Pis78a, Pis78b, Pis83a, Pis83b] and references therein); it is shown to be deeply related to the theory of random Fourier series, metric entropy condition, multiplier spaces as well as some other topics in harmonic analysis and Banach space theory.

The development of similar subjects in more general setting goes naturally into two lines. The first one is the theory of Sidon sets in the setting of a non-abelian compact group $G$; these sets are special subsets of irreducible representations of $G$. This generalized notion of Sidon sets was firstly introduced in [FTR66, HR70] and then has seen many links to the harmonic analysis and probability theory as in the abelian case (see [Boż74, MP81]). The other line of development concerns subsets of an arbitrary non-abelian discrete group $\Gamma$ with related "functions" in its group von Neumann algebra $V N(\Gamma)$, such as in [Pic73, FT77, Boż81]. The latter is more complicated and the behavior of lacunarity is closely related to the amenability of the discrete group, which also involves some tools from the operator space theory and leads to interesting topics in the study of completely bounded $L^{p}$-Fourier multipliers in abstract harmonic analysis ([Har99, Pis95]).

In the both directions mentioned above, the Sidon sets admit many seemingly non-related characterizations, and enjoy some special relations with $\Lambda(p)$-sets, multipliers, etc.. Moreover, many basic properties of Sidon sets in the two different settings have quite similar formulations. This gives rise to a natural motivation to find a more general framework, say Woronowicz's compact quantum groups, to unify these developments from different viewpoints, and to seek new aspects of the theory for these objects. In fact, based on the Woronowicz-Peter-Weyl theory for compact quantum groups, we may discuss similar questions for Sidon sets in the quantum group setting. Note that a priori in this general view, a discrete group $\Gamma$ is regarded as the set of irreducible representations of the dual compact quantum group $\mathbb{G}=\hat{\Gamma}$, and the amenability of $\Gamma$ is often interpreted as the coamenability of $\mathbb{G}$. Recently, Blendek and Michaliček [BM13] have obtained

2010 Mathematics Subject Classification. Primary: 20G42, 46L89. Secondary: 43A46, 46L52.

Key words and phrases. Compact quantum group, Fourier series, Fourier multipliers, Sidon sets, $\Lambda(p)$-sets. 
some partial results towards this direction, which we will significantly complete and improve in Section 4.

In this paper we introduce and develop some important notions and properties related to lacunarities in the framework of compact quantum groups. The subjects that we address are as follows:

(1) Generalizations and characterizations of Sidon sets. With some preliminary work on Fourier series, we introduce in Section 3 the notion of Sidon sets for a compact quantum group and as an analogue of the classical case, we give some first characterizations of Sidon sets via interpolation of Fourier series of bounded functionals or $L^{1}$-functions (Theorem 3.3). Theorem 3.3 answers in particular a basic question in the study on lacunarity of discrete groups, raised in [FT77, Pic73], that is, the equivalence between strong Sidon sets and Sidon sets in discrete groups in the sense of [Pic73]. In fact, in the latter article Picardello defined two different kinds of lacunary sets that he called strong Sidon sets and Sidon sets in discrete groups. He proved the equivalence between these two notions for amenable discrete groups, but the non-amenable case has been left open. Here our approach is different from the classical ones in [HR70, Pic73]. Our argument is simpler and avoids the use of coamenability as in [Pic73]. It as well applies to the general quantum setting. We show as well that the Sidon property is stable under Cartesian/free products of compact quantum groups. Apart from the above approach, there are indeed various viewpoints on generalizations of Sidon sets for non-coamenable compact quantum groups, which lead to different types of lacunarities such as weak Sidon sets and unconditional Sidon sets. In Theorem 3.15 we will discuss the relations between these various notions and prove the equivalence among them for coamenable compact quantum groups.

(2) $\Lambda(p)$-sets and $L^{p}$-Fourier multipliers. We discuss the equivalence between $\Lambda(p)$-sets and interpolation sets of bounded $L^{p}$-Fourier multipliers for a compact quantum group (Theorem 4.7), generalizing the previous work [Har99]. Our investigation also leads to some facts which are hidden in the classical cases: we show that the restriction of the modular element $Q$ of the dual quantum group on a $\Lambda(p)$-set must be bounded (Proposition 4.5), and that the class of $\Lambda(p)$-sets on a compact quantum group is independent of the interpolation parameter in the construction of noncommutative $L^{p}$-spaces (Proposition 4.14).

(3) Relations between Sidon sets and $\Lambda(p)$-sets. For a compact group or (the dual of) a discrete group, a simple (but non-trivial) argument shows that any Sidon set $\mathbf{E}$ is of type $\Lambda(p)$ for $1<$ $p<\infty$, which indeed means that any two norms \|\|$_{p},\|\|_{p^{\prime}}$ for $1<p, p^{\prime}<\infty$ are equivalent on the subspace of polynomials with Fourier series supported in E. The case for a general compact quantum group turns out to be more difficult. A first attempt was made by Blendek and Michaliček [BM13], who showed in 2013 that if $\mathbb{G}$ is a compact quantum group of Kac type and if the Sidon set E satisfies some other special lacunarity conditions, then the norms \|\|$_{1}$ and \|\|$_{2}$ are equivalent on the subspace of central polynomials related to $\mathbf{E}$. In this paper we give a shorter argument which completely solves the problem by showing that any Sidon set for an arbitrary compact quantum group is a $\Lambda(p)$-set for $1<p<\infty$ (see Theorem 4.8 and Corollary 4.9).

(4) Existence of $\Lambda(p)$-sets. We prove in Theorem 4.15 that any infinite subset of irreducible representations with uniformly bounded dimensions admits an infinite $\Lambda(p)$-set. The argument is based on the general fact that any infinite uniformly bounded orthogonal system with respect to a normal faithful state in a von Neumann algebra (including type III) admits an infinite $\Lambda(p)$-set. Since this result may be of independent interest, we include a proof in the appendix. On the other hand, contrary to the classical case, we may find some nice central $\Lambda(4)$-sets for $\mathrm{SU}_{q}(2)$ with $0<q<1$. This will be given in Proposition 4.16.

(5) Central Sidon sets. We also investigate in Section 5 some basic notions and facts concerning central Sidon sets for compact quantum groups. Our argument includes some new characterizations of compact quantum groups of Kac type via bounded central functionals or conditional expectations onto central functions. We show that any two compact quantum groups with the same fusion rules and the same dimension functions have identical central Sidon sets. This gives many examples of central Sidon sets for compact quantum groups via monoidal equivalence, DrinfeldJimbo deformations, etc. As a corollary, we see that the Drinfeld-Jimbo $q$-deformation of any 
compact simply connected semi-simple Lie group (e.g. $\left.\mathrm{SU}_{q}(2)\right)$ does not admit any infinite Sidon set.

We would also like to remark that many results mentioned above rely on some very elementary properties of bounded $L^{p}$-Fourier multipliers on compact quantum groups, which have not been discussed so far in literature. The first property concerns the question how to construct a left bounded $L^{p}$-multiplier from a right bounded $L^{p}$-multiplier, and the second one concerns the inequality comparing the norms $\|a\|_{\ell^{\infty}(\hat{\mathbb{G}})}$ and $\|a\|_{\mathrm{M}\left(L^{p}(\mathbb{G})\right)}$. These questions are easy to deal with for completely bounded $L^{\infty}$-multipliers, but it seems that they are not obvious for other cases. Although the completely bounded $L^{\infty}$-multipliers, instead of bounded $L^{\infty}$-multipliers, are more natural objects for studying harmonic analysis on quantum groups, the questions above are still natural and elementary for studying $L^{p}$-Fourier analysis on quantum groups, especially for $p<\infty$. As a result we include a detailed argument on these basic facts (Lemma 2.5, Proposition 2.7) in Section 2, which are also frequently used in other proofs of this paper.

It would also be interesting to study the completely bounded version of Sidon sets or $\Lambda(p)$-sets. We refer to Pisier's work [Pis95] for completely bounded lacunarity in discrete groups. We have not studied here this topic; but we will pursue it elsewhere. In fact, our argument on $L^{p}$-Fourier multipliers in Section 2, and the estimation of modular elements in Section 4, will be helpful for the study towards this direction.

\section{Preliminaries}

\subsection{Compact quantum groups.}

1.1.1. Basic notions. Let us first recall some well-known definitions and properties concerning compact quantum groups. We refer to [Wor98] and [MVD98] for more details.

Definition 1.1. Let $A$ be a unital $\mathrm{C}^{*}$-algebra. If there exists a unital $*$-homomorphism $\Delta: A \rightarrow$ $A \otimes A$ such that $(\Delta \otimes \iota) \Delta=(\iota \otimes \Delta) \Delta$ and

$$
\{\Delta(a)(1 \otimes b): a, b \in A\} \quad \text { and } \quad\{\Delta(a)(b \otimes 1): a, b \in A\}
$$

are linearly dense in $A \otimes A$, then $(A, \Delta)$ is called a compact quantum group and $\Delta$ is called the comultiplication on $A$. We denote $\mathbb{G}=(A, \Delta)$ and $A=C(\mathbb{G})$.

Any compact quantum group $\mathbb{G}$ admits a unique Haar state $h$ on $C(\mathbb{G})$ such that for all $x \in C(\mathbb{G})$,

$$
(h \otimes \iota) \circ \Delta(x)=h(x) 1=(\iota \otimes h) \circ \Delta(x) .
$$

Consider an element $u \in C(\mathbb{G}) \otimes B(H)$, where $H$ is a Hilbert space with $\operatorname{dim} H=n$. We identify $C(\mathbb{G}) \otimes B(H)=\mathbb{M}_{n}(C(\mathbb{G}))$ and write $u=\left[u_{i j}\right]_{i, j=1}^{n}$. The matrix $u$ is called an $n$-dimensional representation of $\mathbb{G}$ if for all $j, k=1, \ldots, n$ we have

$$
\Delta\left(u_{j k}\right)=\sum_{p=1}^{n} u_{j p} \otimes u_{p k} .
$$

Denote by $\operatorname{Irr}(\mathbb{G})$ the set of unitary equivalence classes of irreducible finite-dimensional unitary representations of $\mathbb{G}$. Also denote by $\operatorname{Rep}(\mathbb{G})$ the set of unitary equivalence classes of (not necessarily irreducible) finite-dimensional unitary representations of $\mathbb{G}$. For each $\pi \in \operatorname{Irr}(\mathbb{G})$, we fix a representative $u^{(\pi)} \in C(\mathbb{G}) \otimes B\left(H_{\pi}\right)$ of the class $\pi$ where $H_{\pi}$ is the finite dimensional Hilbert space on which $u^{(\pi)}$ acts. In the sequel we write $n_{\pi}=\operatorname{dim} \pi=\operatorname{dim} H_{\pi}$ for $\pi \in \operatorname{Irr}(\mathbb{G})$.

For $\pi, \pi^{\prime} \in \operatorname{Irr}(\mathbb{G})$, define the tensor product representation $\pi \otimes \pi^{\prime}$ on $H_{\pi} \otimes H_{\pi^{\prime}}$ by

$$
u^{\left(\pi \otimes \pi^{\prime}\right)}=\sum_{i, j, k, l} u_{i j}^{(\pi)} u_{k l}^{\left(\pi^{\prime}\right)} \otimes e_{i j}^{(\pi)} \otimes e_{k l}^{\left(\pi^{\prime}\right)},
$$

where $e_{i j}^{(\pi)}, e_{k l}^{\left(\pi^{\prime}\right)}$ denotes the matrix units of $B\left(H_{\pi}\right)$ and $B\left(H_{\pi^{\prime}}\right)$ respectively. For each $\pi \in \operatorname{Irr}(\mathbb{G})$, there exists a unique $\bar{\pi} \in \operatorname{Irr}(\mathbb{G})$ such that the trivial representation $1 \in \operatorname{Irr}(\mathbb{G})$ is a subrepresentation of $\pi \otimes \bar{\pi}$. We call $\bar{\pi}$ the adjoint of $\pi$. The notions of tensor product and adjoint then can be extended to all elements in $\operatorname{Rep}(\mathbb{G})$ by decomposing representations into irreducible ones. 
For $\pi \in \operatorname{Rep}(\mathbb{G})$, the character of $\pi$ is the element $\chi_{\pi}=\sum_{i=1}^{n_{\pi}} u_{i i}^{(\pi)} \in C(\mathbb{G})$. One can show that the definition does not depend on the representative matrix $u^{(\pi)}$. For $\pi, \pi^{\prime} \in \operatorname{Rep}(\mathbb{G})$, we have

$$
\chi_{\pi \oplus \pi^{\prime}}=\chi_{\pi}+\chi_{\pi^{\prime}}, \quad \chi_{\pi \otimes \pi^{\prime}}=\chi_{\pi} \chi_{\pi^{\prime}}, \quad \chi_{\bar{\pi}}=\chi_{\pi}^{*} .
$$

Denote $\operatorname{Pol}(\mathbb{G})=\operatorname{span}\left\{u_{i j}^{(\pi)}: u^{(\pi)}=\left[u_{i j}^{(\pi)}\right]_{i, j=1}^{n_{\pi}}, \pi \in \operatorname{Irr}(\mathbb{G})\right\}$. This is a dense subalgebra of $C(\mathbb{G})$. Consider the GNS representation $\left(\pi_{h}, H_{h}\right)$ of the Haar state $h$, then $\operatorname{Pol}(\mathbb{G})$ can be viewed as a subalgebra of $B\left(H_{h}\right)$. Define $C_{r}(\mathbb{G})$ (resp., $L^{\infty}(\mathbb{G})$ ) to be the $\mathrm{C}^{*}$-algebra (resp., the von Neumann algebra) generated by $\operatorname{Pol}(\mathbb{G})$ in $B\left(H_{h}\right)$. Then $h$ extends to a normal faithful state on $L^{\infty}(\mathbb{G})$. On the other hand, we may equip the following $C^{*}$-norm on $\operatorname{Pol}(\mathbb{G})$,

$$
\|x\|_{u}=\sup \left\{p(x): p \text { is a } C^{*} \text {-seminorm on } \operatorname{Pol}(\mathbb{G})\right\}, \quad x \in \operatorname{Pol}(\mathbb{G}) .
$$

Then the corresponding completion of $\operatorname{Pol}(\mathbb{G})$ is a unital $\mathrm{C}^{*}$-algebra, denoted by $C_{u}(\mathbb{G})$.

It is known that there exists a linear antihomomorphism $S$ on $\operatorname{Pol}(\mathbb{G})$, called the antipode of $\mathbb{G}$, determined by

$$
S\left(u_{i j}^{(\pi)}\right)=\left(u_{j i}^{(\pi)}\right)^{*}, \quad u^{(\pi)}=\left[u_{i j}^{(\pi)}\right]_{i, j=1}^{n_{\pi}}, \pi \in \operatorname{Irr}(\mathbb{G}) .
$$

Also, let $\epsilon$ be the counit of $\operatorname{Pol}(\mathbb{G})$, i.e., the linear functional defined by

$$
\epsilon\left(u_{i j}^{(\pi)}\right)=\delta_{i j}, \quad u^{(\pi)}=\left[u_{i j}^{(\pi)}\right]_{i, j=1}^{n_{\pi}}, \pi \in \operatorname{Irr}(\mathbb{G}) .
$$

Let $\left(\mathbb{G}_{i}: i \in I\right)$ be a family of compact quantum groups. It is shown in [Wan95b] that there exists a compact quantum group, denoted by $\prod_{i \in I} \mathbb{G}_{i}$, such that the algebra $C_{r}\left(\prod_{i \in I} \mathbb{G}_{i}\right)$ is the minimal tensor product of the $\mathrm{C}^{*}$-algebras $C_{r}\left(\mathbb{G}_{i}\right)$, and the comultiplication on $\prod_{i \in I} \mathbb{G}_{i}$ is induced by those on $\mathbb{G}_{i}$ 's. We call $\prod_{i \in I} \mathbb{G}_{i}$ the Cartesian product of $\left(\mathbb{G}_{i}\right)$. Each irreducible representation $\pi \in \operatorname{Irr}\left(\mathbb{G}_{i}\right)$ can be naturally viewed as an irreducible representation of $\prod_{i \in I} \mathbb{G}_{i}$, still denoted by $\pi$. In this way we may write $\operatorname{Irr}\left(\prod_{i \in I} \mathbb{G}_{i}\right)=\left\{\otimes_{i \in I} \pi_{i}: \pi_{i} \in \operatorname{Irr}\left(\mathbb{G}_{i}\right), i \in I\right\}$. On the other hand, according to [Wan95a], we may construct a compact quantum group denoted by $\hat{*}_{i \in I} \mathbb{G}_{i}$ and called the dual free product of $\left(\mathbb{G}_{i}\right)$, such that the algebra $C_{r}\left(\hat{*}_{i \in I} \mathbb{G}_{i}\right)$ is the reduced free product of the $\mathrm{C}^{*}$-algebras $C_{r}\left(\mathbb{G}_{i}\right)$ associated to the Haar states of $\mathbb{G}_{i}$. Again, each irreducible representation $\pi \in \operatorname{Irr}\left(\mathbb{G}_{i}\right)$ can be naturally viewed as an irreducible representation of $\hat{*}_{i \in I} \mathbb{G}_{i}$, still denoted by $\pi$.

1.1.2. Modular properties of the Haar state. It is well-known that for each $\pi \in \operatorname{Irr}(\mathbb{G})$ there exists a unique positive invertible operator $Q_{\pi} \in B\left(H_{\pi}\right)$ with $\operatorname{Tr}\left(Q_{\pi}\right)=\operatorname{Tr}\left(Q_{\pi}^{-1}\right):=d_{\pi}$ that intertwines $u^{(\pi)}$ and $\left(S^{2} \otimes \iota\right)\left(u^{(\pi)}\right)$. Then the Haar state can be calculated as follows,

$$
h\left(u_{i j}^{(\pi)}\left(u_{l m}^{\left(\pi^{\prime}\right)}\right)^{*}\right)=\delta_{\pi \pi^{\prime}} \delta_{i l} \frac{\left(Q_{\pi}\right)_{m j}}{d_{\pi}}, \quad h\left(\left(u_{i j}^{(\pi)}\right)^{*} u_{l m}^{\left(\pi^{\prime}\right)}\right)=\delta_{\pi \pi^{\prime}} \delta_{j m} \frac{\left(Q_{\pi}^{-1}\right)_{l i}}{d_{\pi}},
$$

where $\pi^{\prime} \in \operatorname{Irr}(\mathbb{G}), 1 \leq i, j \leq n_{\pi}, 1 \leq l, m \leq n_{\pi^{\prime}}$. The number $d_{\pi}$ is called the quantum dimension of $\pi$. The quantum group $\mathbb{G}$ is said to be of Kac type if $Q_{\pi}=\operatorname{Id}_{\pi}$ for all $\pi \in \operatorname{Irr}(\mathbb{G})$. The Woronowicz characters on $\operatorname{Pol}(\mathbb{G})$ are defined as

$$
f_{z}\left(u_{i j}^{(\pi)}\right)=\left(Q_{\pi}^{z}\right)_{i j}, \quad z \in \mathbb{C}, \pi \in \operatorname{Irr}(\mathbb{G}), 1 \leq i, j \leq n_{\pi} .
$$

We denote

$$
\Delta^{(2)}:=(\Delta \otimes \iota) \Delta=(\iota \otimes \Delta) \Delta .
$$

The modular automorphism group of the Haar state $h$ on $L^{\infty}(\mathbb{G})$ is determined by the following formula:

in other words,

$$
\sigma_{z}(x)=\left(f_{\mathrm{i} z} \otimes \iota \otimes f_{\mathrm{i} z}\right) \Delta^{(2)}(x), \quad x \in \operatorname{Pol}(\mathbb{G}), z \in \mathbb{C},
$$

$$
\left(\sigma_{z} \otimes \iota\right)\left(u^{(\pi)}\right)=\left(1 \otimes Q_{\pi}^{\mathrm{i} z}\right) u^{(\pi)}\left(1 \otimes Q_{\pi}^{\mathrm{i} z}\right), \quad \pi \in \operatorname{Irr}(\mathbb{G}) .
$$

The antipode $S$ has the following polar decomposition

$$
S=R \circ \tau_{-\frac{i}{2}}=\tau_{-\frac{i}{2}} \circ R,
$$

where $R$ is a $*$-antiautomorphism of $C_{r}(\mathbb{G})$ and $\left(\tau_{z}\right)_{z \in \mathbb{C}}$ is the analytic extension of the oneparameter group $\left(\tau_{t}\right)_{t \in \mathbb{R}}$ of $*$-automorphisms defined as

$$
\tau_{z}(x)=\left(f_{\mathrm{i} z} \otimes \iota \otimes f_{-\mathrm{i} z}\right) \Delta^{(2)}(x)
$$


for $x \in \operatorname{Pol}(\mathbb{G})$, or in other words,

$$
\left(\tau_{z} \otimes \iota\right)\left(u^{(\pi)}\right)=\left(1 \otimes Q_{\pi}^{\mathrm{i} z}\right) u^{(\pi)}\left(1 \otimes Q_{\pi}^{-\mathrm{i} z}\right), \quad \pi \in \operatorname{Irr}(\mathbb{G}),
$$

and moreover

$$
S^{2}=\tau_{-\mathrm{i}}, \quad \Delta \circ R=\Sigma \circ(R \otimes R) \circ \Delta .
$$

The dual quantum group $\hat{\mathbb{G}}$ of $\mathbb{G}$ is defined via its "algebras of functions",

$$
c_{0}(\hat{\mathbb{G}})=\oplus_{\pi \in \operatorname{Irr}(\mathbb{G})}^{c_{0}} B\left(H_{\pi}\right), \quad \ell^{\infty}(\hat{\mathbb{G}})=\oplus_{\pi \in \operatorname{Irr}(\mathbb{G})} B\left(H_{\pi}\right),
$$

where $\oplus_{\pi} B\left(H_{\pi}\right)$ refers to the direct sum of $B\left(H_{\pi}\right)$, i.e. the bounded families $\left(x_{\pi}\right)_{\pi}$ with each $x_{\pi}$ in $B\left(H_{\pi}\right)$, and $\oplus_{\pi \in \operatorname{Irr}(\mathbb{G})}^{c_{0}} B\left(H_{\pi}\right)$ corresponds to the subalgebra of bounded families converging to 0 at infinity. Also set $c_{c}(\hat{\mathbb{G}})$ to be the corresponding algebraic direct sum and denote by $\prod_{\pi} B\left(H_{\pi}\right)$ the usual Cartesian product. We will not recall the full quantum group structure on $\hat{\mathbb{G}}$ as we do not need it in the following. We only remark that the (left) Haar weight $\hat{h}$ on $\hat{\mathbb{G}}$ can be explicitly given by (see e.g. [VD96, Section 5])

$$
\hat{h}: \ell^{\infty}(\hat{\mathbb{G}}) \ni x \mapsto \sum_{\pi \in \operatorname{Irr}(\mathbb{G})} d_{\pi} \operatorname{Tr}\left(Q_{\pi} p_{\pi} x\right),
$$

where $p_{\pi}$ is the projection onto $H_{\pi}$ and $\operatorname{Tr}$ denotes the usual trace on $B\left(H_{\pi}\right)$ for each $\pi$.

1.1.3. Coamenability. Consider a compact quantum group $\mathbb{G}$. We say that $\mathbb{G}$ is coamenable if the counit $\epsilon: \operatorname{Pol}(\mathbb{G}) \rightarrow \mathbb{C}$ extends to a state on $C_{r}(\mathbb{G})$. On the other hand, for linear functionals $\varphi, \varphi^{\prime}$ on $\operatorname{Pol}(\mathbb{G})$, we define the convolution product

$$
\varphi \star \varphi^{\prime}=\left(\varphi \otimes \varphi^{\prime}\right) \circ \Delta .
$$

Then it is easy to see that

$$
\left\|\varphi_{1} \star \varphi_{2}\right\| \leq\left\|\varphi_{1}\right\|\left\|\varphi_{2}\right\|
$$

where the norm is induced from $C_{r}(\mathbb{G})^{*}$ or $L^{\infty}(\mathbb{G})^{*}$, when the respective functionals admit bounded extensions. Equipped with this convolution product, the predual space $L^{\infty}(\mathbb{G})_{*}$ forms a Banach algebra. The following characterization of coamenability is given in [BT03, Theorem 3.1].

Proposition 1.2. A compact quantum group $\mathbb{G}$ is coamenable if and only if the Banach algebra $L^{\infty}(\mathbb{G})_{*}$ has a bounded right approximate unit with norm not more than 1 , and if and only if the identity map on $\operatorname{Pol}(\mathbb{G})$ extends to a *-isomorphism from $C_{u}(\mathbb{G})$ to $C_{r}(\mathbb{G})$.

We remark that any compact group $G$ or compact quantum group $\mathbb{G}$ with an amenable discrete group $\Gamma$ as the dual quantum group, is coamenable.

1.1.4. Drinfeld-Jimbo deformation, quantum $\mathrm{SU}(N)$ groups. Let $G$ be a simply connected semisimple compact Lie group. It follows from the work of Levendorskii and Soibelman [LS91, Soi90] that given any $q>0$ one can define a compact quantum group $G_{q}$, called the Drinfeld-Jimbo $q$ deformation of $G$ such that the fusion rules, the classical dimension function and the coamenablity do not depend on $q$. More precisely, we may state the following property (see for example [NT13, Theorem 2.4.7], [Ban99] and references therein).

Proposition 1.3. Let $0<q<1$.

(1) There exists a bijection $\Phi: \operatorname{Rep}(G) \rightarrow \operatorname{Rep}\left(G_{q}\right)$ such that

$$
\Phi\left(\pi \otimes \pi^{\prime}\right)=\Phi(\pi) \otimes \Phi\left(\pi^{\prime}\right), \quad \Phi\left(\oplus_{i \in I} \pi_{i}\right)=\oplus_{i \in I} \Phi\left(\pi_{i}\right), \quad \pi, \pi^{\prime}, \pi_{i} \in \operatorname{Rep}(G)
$$

and $\operatorname{dim} \Phi(\pi)=\operatorname{dim} \pi$ for all $\pi \in \operatorname{Irr}(G)$.

(2) $G_{q}$ is coamenable.

Take $G$ to be the special unitary group $\mathrm{SU}(N)$ of degree $N$. We denote by $\mathrm{SU}_{q}(N)$ the compact quantum group $G_{q}$ for $0<q<1$. Let us recall some facts of the representation theory of $\mathrm{SU}_{q}(2)$. The elements of $\operatorname{Irr}\left(\mathrm{SU}_{q}(2)\right)$ can be indexed by $n \in \mathbb{N} \cup\{0\}$ and each representation $u^{(n)}$ is of 
dimension $n+1$. The associated matrix $Q_{n}$ in (1.2) can be represented as a diagonal under some appropriate basis (see for example Theorem 17 in [KS97, Sect.4.3.2]):

$$
Q_{n}=\left[\begin{array}{ccccc}
q^{-n} & & & & \\
& q^{-n+2} & & & \\
& & \ddots & & \\
& & & q^{n-2} & \\
& & & & q^{n}
\end{array}\right] \text {. }
$$

Write $\chi_{n}=\sum_{i} u_{i i}^{(n)}$ to be the character of $u^{(n)}$. We recall the property below. See for example Proposition 6.2.10 in [Tim08] for the proof.

Proposition 1.4. (1) For $m, m^{\prime} \in \mathbb{N} \cup\{0\}, \chi_{m} \chi_{m^{\prime}}=\chi_{\left|m-m^{\prime}\right|}+\chi_{\left|m-m^{\prime}\right|+1}+\cdots+\chi_{m+m^{\prime}}$;

(2) For $n \in \mathbb{N} \cup\{0\}, \chi_{n}=\chi_{n}^{*}$.

1.2. Noncommutative $L^{p}$-spaces. In this subsection we recall some basic definitions and facts on noncommutative $L^{p}$-spaces. We refer to [Tak02, Tak03] for the theory of von Neumann algebras and to [PX03] for more details on noncommutative $L_{p}$-spaces. In this paper we will mainly use the construction via interpolation [Kos84] and we refer to [BL76] for all notions and notation from interpolation theory used below.

Let $\mathcal{M}$ be a von Neumann algebra equipped with a distinguished normal faithful state $\varphi$. Denote by $\mathcal{M}_{*}$ the predual space of $\mathcal{M}$. Define $L^{1}(\mathcal{M}, \varphi)=\mathcal{M}_{*}$ and $L^{\infty}(\mathcal{M}, \varphi)=\mathcal{M}$. We identify $\mathcal{M}$ as a subspace of $\mathcal{M}_{*}$ by the following injection

$$
j: \mathcal{M} \rightarrow \mathcal{M}_{*}, \quad j(x)=x \varphi:=\varphi(\cdot x), \quad x \in \mathcal{M} .
$$

It is known that $j$ is a contractive injection with dense image. In this way we may view $\left(\mathcal{M}, \mathcal{M}_{*}\right)$ as a compatible pair of Banach spaces and for $1<p<\infty$, we introduce the corresponding noncommutative $L^{p}$-space as

$$
L^{p}(\mathcal{M}, \varphi)=\left(\mathcal{M}, \mathcal{M}_{*}\right)_{1 / p}
$$

where $(\cdot, \cdot)_{1 / p}$ denotes the complex interpolation space. Denote by $\|\cdot\|_{p}$ the norm on $L^{p}(\mathcal{M}, \varphi)$. Let $H_{\varphi}$ be the Hilbert space in the GNS construction induced by $\varphi$. Then

$$
L^{2}(\mathcal{M}, \varphi)=H_{\varphi}
$$

with equal norms.

Another useful and equivalent construction of noncommutative $L^{p}$-spaces is given by Haagerup. We refer to [Ter81, PX03, HJX10] for details. For the convenience of the reader, we will not formulate the precise definition but rather cite here several basic properties which will be sufficient for later use.

Assume that the von Neumann algebra $\mathcal{M}$ acts on a Hilbert space $H$ and denote by $\sigma=\sigma^{\varphi}$ the modular automorphism group of $\varphi$. For $1 \leq p \leq \infty$, denote by $L^{p, \mathrm{H}}(\mathcal{M}, \varphi)$ the corresponding Haagerup $L^{p}$-space with norm $\|\cdot\|_{p, H}$. Recall that each element in $L^{p, H}(\mathcal{M}, \varphi)$ is realized as a densely defined operator on $L^{2}(\mathbb{R}, H)$ and the usual Hölder inequality also holds for these noncommutative $L^{p}$-spaces in this sense. Also note that $\mathcal{M}$ can be identified with $L^{\infty, \mathrm{H}}(\mathcal{M}, \varphi)$.

Let $D$ be the density operator associated to $\varphi$ and $\operatorname{tr}$ be the trace on $L^{1, \mathrm{H}}(\mathcal{M}, \varphi)$. We recall that $D$ is a distinguished invertible positive selfadjoint operator on $L^{2}(\mathbb{R}, H)$ and tr is a distinguished positive functional on $L^{1, \mathrm{H}}(\mathcal{M}, \varphi)$, which enjoy the following properties.

Proposition 1.5. (1) For all $x \in \mathcal{M}, t \in \mathbb{R}, \sigma_{t}(x)=D^{\mathrm{i} t} x D^{-\mathrm{i} t}$;

(2) Let $1 \leq p, q \leq \infty$ be such that $1 / p+1 / q=1$. Then for $x \in L^{p, \mathrm{H}}(\mathcal{M}, \varphi), y \in L^{q, \mathrm{H}}(\mathcal{M}, \varphi)$, we have $x y, y x \in L^{1, \mathrm{H}}(\mathcal{M}, \varphi)$ and $\operatorname{tr}(x y)=\operatorname{tr}(y x)$;

(3) $D \in L^{1, \mathrm{H}}(\mathcal{M}, \varphi)$ and $\varphi(x)=\operatorname{tr}(x D)$ for $x \in \mathcal{M}$;

(4) For $x \in L^{1, \mathrm{H}}(\mathcal{M}, \varphi), \operatorname{tr}(|x|)=\|x\|_{1, \mathrm{H}}$. For $x \in L^{p, \mathrm{H}}(\mathcal{M}, \varphi)$ with $1 \leq p<\infty$, we have

$$
|x|^{p} \in L^{1, \mathrm{H}}(\mathcal{M}, \varphi), \quad\|x\|_{p, \mathrm{H}}=\left\|x^{*}\right\|_{p, \mathrm{H}}=\||x|\|_{p, \mathrm{H}}=\left\||x|^{p}\right\|_{1, \mathrm{H}}^{1 / p} ;
$$

and for $x \in \mathcal{M}, 1 \leq p \leq p^{\prime} \leq \infty$, we have

$$
x D^{1 / p} \in L^{p, \mathrm{H}}(\mathcal{M}, \varphi), \quad x D^{1 / p^{\prime}} \in L^{p^{\prime}, \mathrm{H}}(\mathcal{M}, \varphi), \quad\left\|x D^{1 / p}\right\|_{p, \mathrm{H}} \leq\left\|x D^{1 / p^{\prime}}\right\|_{p^{\prime}, \mathrm{H}} ;
$$


(5) ([Jun02, (1.3)]) For $1 \leq p<\infty$ and for $0 \leq x \leq y \in L^{p, \mathrm{H}}(\mathcal{M})$, we have $\|x\|_{p, \mathrm{H}} \leq\|y\|_{p, \mathrm{H}}$.

In this paper we will frequently identify the two $L^{p}$-spaces via the following isomorphism.

Proposition 1.6 ([Kos84, Sect.9]). For all $1 \leq p \leq \infty$, the map

$$
j^{p}: x \mapsto x D^{1 / p}, x \in \mathcal{M}
$$

extends to an isometry from $L^{p}(\mathcal{M}, \varphi)$ onto $L^{p, \mathrm{H}}(\mathcal{M}, \varphi)$.

We will need the noncommutative Khintchine inequality for Rademacher sequences. Let $2 \leq$ $p<\infty$. For a finitely supported sequence $\left(x_{n}\right)_{1 \leq n \leq N} \subset L^{p, \mathrm{H}}(\mathcal{M}, \varphi)$, we introduce the notation

$$
\left\|\left(x_{n}\right)\right\|_{L^{p}\left(\mathcal{M} ; \ell_{c}^{2}\right)}=\left\|\left(\sum_{n}\left|x_{n}\right|^{2}\right)^{1 / 2}\right\|_{p, \mathrm{H}}, \quad\left\|\left(x_{n}\right)\right\|_{L^{p}\left(\mathcal{M} ; \ell_{r}^{2}\right)}=\left\|\left(\sum_{n}\left|x_{n}^{*}\right|^{2}\right)^{1 / 2}\right\|_{p, \mathrm{H}},
$$

and write

$$
\left\|\left(x_{n}\right)\right\|_{C R_{p}\left[L^{p}(\mathcal{M})\right]}=\max \left\{\left\|\left(x_{n}\right)\right\|_{L^{p}\left(\mathcal{M} ; \ell_{c}^{2}\right)},\left\|\left(x_{n}\right)\right\|_{L^{p}\left(\mathcal{M} ; \ell_{r}^{2}\right)}\right\} .
$$

By Proposition 1.5 and the triangle inequality, we see easily that

$$
\left\|\left(x_{n}\right)\right\|_{C R_{p}\left[L^{p}(\mathcal{M})\right]} \leq\left(\sum_{n}\left\|x_{n}\right\|_{L^{p, H}(\mathcal{M}, \varphi)}^{2}\right)^{1 / 2} .
$$

Denote by $\left(\varepsilon_{n}\right)_{n \geq 1}$ a Rademacher sequence on a probability space $(\Omega, P)$, i.e., an independent sequence of random variables with $P\left(\varepsilon_{n}=1\right)=P\left(\varepsilon_{n}=-1\right)=1 / 2$ for all $n$. The following noncommutative Khintchine inequality for Haagerup's $L^{p}$-spaces is given in [JX03, Theorem 3.4].

Theorem 1.7. There exists an absolute constant $C>0$ such that for all $2 \leq p<\infty$ and all finitely supported sequences $\left(x_{n}\right)$ in $L^{p, \mathrm{H}}(\mathcal{M}, \varphi)$, we have

$$
\left\|\left(x_{n}\right)\right\|_{C R_{p}\left[L^{p}(\mathcal{M})\right]} \leq\left(\int_{\Omega}\left\|\sum_{n} \varepsilon_{n}(\omega) x_{n}\right\|_{L^{p, \mathrm{H}(\mathcal{M}, \varphi)}}^{p} d P(\omega)\right)^{1 / p} \leq C \sqrt{p}\left\|\left(x_{n}\right)\right\|_{C R_{p}\left[L^{p}(\mathcal{M})\right]} .
$$

Consequently $L^{p, \mathrm{H}}(\mathcal{M}, \varphi)$ is of type 2 , i.e., for the above sequences we have

$$
\left(\int_{\Omega}\left\|\sum_{n} \varepsilon_{n}(\omega) x_{n}\right\|_{L^{p, \mathrm{H}(\mathcal{M}, \varphi)}}^{p} d P(\omega)\right)^{1 / p} \leq C \sqrt{p}\left(\sum_{n}\left\|x_{n}\right\|_{L^{p, \mathrm{H}}(\mathcal{M}, \varphi)}^{2}\right)^{1 / 2} .
$$

In this paper, we will mainly be interested in the case of $\mathcal{M}=L^{\infty}(\mathbb{G})$ for a compact quantum group $\mathbb{G}$. Let $h$ be the Haar state on $\mathbb{G}$. Throughout the paper, for any $1 \leq p \leq \infty$ we will use the notation $L^{p}(\mathbb{G}):=L^{p}\left(L^{\infty}(\mathbb{G}), h\right)$ for Kosaki's noncommutative $L^{p}$-spaces and $L^{p, \mathrm{H}}(\mathbb{G}):=$ $L^{p, \mathrm{H}}\left(L^{\infty}(\mathbb{G}), h\right)$ for Haagerup's noncommutative $L^{p}$-spaces introduced above. As is seen in the previous construction, we will in the sequel identify $L^{\infty}(\mathbb{G})$ with a subspace of $L^{1}(\mathbb{G})$ via the embedding

$$
j: x \mapsto x h:=h(\cdot x) .
$$

In particular we define the convolution for $x, x^{\prime} \in L^{\infty}(\mathbb{G})$,

$$
x \star x^{\prime}:=(x h) \star\left(x^{\prime} h\right)\left(\in L^{1}(\mathbb{G})\right) .
$$

It is easy to see that the algebra of polynomials $\operatorname{Pol}(\mathbb{G})$ is a common dense subspace of all $L^{p}$-spaces associated to $\mathbb{G}$ with $1 \leq p<\infty$. In fact we have the following property (see [Jun02, Lemma 2.2]).

Lemma 1.8. Let $A$ be an ultraweakly dense $*$-subalgebra of $\mathcal{M}$. Then for $1 \leq p<\infty, A$ is dense in $L^{p}(\mathcal{M}, \varphi)$ with respect to \|\|$_{p}$.

We will not consider the noncommutative $L^{p}$-spaces associated to discrete quantum groups for a general $p$ in this text, but let us add several words on the special cases $p=1$ and 2 . We consider the dual discrete quantum group $\hat{\mathbb{G}}$ of a compact quantum group $\mathbb{G}$. Define the $L^{1}$-space $\ell^{1}(\hat{\mathbb{G}})$ on $\hat{\mathbb{G}}$ associated to $\hat{h}$ as

$$
\ell^{1}(\hat{\mathbb{G}})=\left\{x \in c_{0}(\hat{\mathbb{G}}):\|x\|_{1}=\sum_{\pi \in \operatorname{Irr}(\mathbb{G})} d_{\pi} \operatorname{Tr}\left(\left|p_{\pi} x Q_{\pi}\right|\right)<\infty\right\} .
$$


By the property of $\operatorname{Tr}$ on $B\left(\oplus_{\pi} H_{\pi}\right)$, it is easy to see that $\ell^{1}(\hat{\mathbb{G}})$ is a Banach space and the injection

$$
j^{\prime}: \ell^{1}(\hat{\mathbb{G}}) \rightarrow \ell^{\infty}(\hat{\mathbb{G}})_{*}, \quad j^{\prime}(x)=x \hat{h}:=\hat{h}(\cdot x), \quad x \in \ell^{1}(\hat{\mathbb{G}})
$$

is an isometric isomorphism. One can easily see that $c_{c}(\hat{\mathbb{G}})$ is a dense subset in $\ell^{1}(\hat{\mathbb{G}})$. Also, we define the space $\ell^{2}(\hat{\mathbb{G}})$ to be the Hilbert space in the GNS construction induced by the Haar weight $\hat{h}$ on $\ell^{\infty}(\hat{\mathbb{G}})$.

\section{FOURIER SERIES AND MULTIPLIERS}

The Fourier transform for locally compact quantum groups has been discussed in [Coo10], [Cas13] and [Kah10]. In the setting of compact quantum groups, we may give a more explicit description. Let a compact quantum group $\mathbb{G}$ be fixed. For a linear functional $\varphi$ on $\operatorname{Pol}(\mathbb{G})$, we define the Fourier transform $\hat{\varphi}=(\hat{\varphi}(\pi))_{\pi \in \operatorname{Irr}(\mathbb{G})} \in \oplus_{\pi} B\left(H_{\pi}\right)$ by

$$
\hat{\varphi}(\pi)=(\varphi \otimes \iota)\left(\left(u^{(\pi)}\right)^{*}\right) \in B\left(H_{\pi}\right), \quad \pi \in \operatorname{Irr}(\mathbb{G}) .
$$

In particular, any $x \in L^{\infty}(\mathbb{G})$ (or $L^{2}(\mathbb{G})$ ) induces a functional $x h:=h(\cdot x)$ on $\operatorname{Pol}(\mathbb{G})$ defined by $y \mapsto h(y x)$, and the Fourier transform $\hat{x}=(\hat{x}(\pi))_{\pi \in \operatorname{Irr}(\mathbb{G})}$ of $x$ is given by

$$
\hat{x}(\pi)=(h(\cdot x) \otimes \iota)\left(\left(u^{(\pi)}\right)^{*}\right) \in B\left(H_{\pi}\right), \quad \pi \in \operatorname{Irr}(\mathbb{G}) .
$$

The above definition is slightly different from that of [Cas13] or [Kah10]. Indeed, we replace the unitary $u^{(\pi)}$ by $\left(u^{(\pi)}\right)^{*}$ in the above formulas. This is just to be compatible with standard definitions in classical analysis on compact groups such as in [Fol95, Section 5.3], and this will not cause any essential difference. We refer to Section 2.2.2 of [Wan16] for some explanation on classical examples in this setting. On the other hand, the notation $\hat{\varphi}$ has a slight conflict with the dual Haar weight $\hat{h}$ on $\hat{\mathbb{G}}$. One can however distinguish them by the elements on which it acts, so we hope that this will not cause any ambiguity for the reader.

Let $\varphi_{1}, \varphi_{2}$ be linear functionals on $\operatorname{Pol}(\mathbb{G})$. Consider their convolution product $\varphi_{1} \star \varphi_{2}=$ $\left(\varphi_{1} \otimes \varphi_{2}\right) \circ \Delta$. We note that for a linear functional $\varphi$ on $\operatorname{Pol}(\mathbb{G})$ and $\pi \in \operatorname{Irr}(\mathbb{G})$,

$$
\left(\varphi \circ S^{-1}\right)^{\wedge}(\pi)=\left(\left(\varphi \circ S^{-1}\right) \otimes \iota\right)\left(\left(u^{(\pi)}\right)^{*}\right)=\left[\varphi\left(u_{i j}^{(\pi)}\right)\right]_{i, j} .
$$

In particular,

$$
\left(\varphi^{*} \circ S^{-1}\right)^{\wedge}(\pi)=\hat{\varphi}(\pi)^{*}
$$

where $\varphi^{*}$ denotes the usual adjoint of $\varphi$, i.e., $\varphi^{*}(x)=\overline{\varphi\left(x^{*}\right)}$. On the other hand, a straightforward calculation shows that

$$
\left(\varphi_{1} \star \varphi_{2}\right)^{\wedge}(\pi)=\hat{\varphi}_{2}(\pi) \hat{\varphi}_{1}(\pi) .
$$

Using the property of the antipode $S$ we may also show (cf. [VD07, Proposition 2.2], [Wan16, $(2.14)])$ that for a linear functional $\varphi$ on $L^{\infty}(\mathbb{G})$ and $x \in L^{\infty}(\mathbb{G}), \pi \in \operatorname{Irr}(\mathbb{G})$,

$$
((\iota \otimes \varphi) \Delta(x))^{\wedge}(\pi)=\left(\varphi \circ S^{-1}\right)^{\wedge}(\pi) \hat{x}(\pi), \quad((\varphi \otimes \iota) \Delta(x))^{\wedge}(\pi)=\hat{x}(\pi)(\varphi \circ S)^{\wedge}(\pi) .
$$

Using (1.5) and (1.6) we may rewrite the second equality above as

$$
((\varphi \otimes \iota) \Delta(x))^{\wedge}(\pi) Q_{\pi}=\hat{x}(\pi) Q_{\pi}\left(\varphi \circ S^{-1}\right)^{\wedge}(\pi) .
$$

In the sequel let $\mathcal{F}: f \mapsto \hat{f}$ denote the Fourier transform.

Proposition 2.1. $\mathcal{F}$ is a contraction from $L^{\infty}(\mathbb{G})^{*}$ to $\ell^{\infty}(\hat{\mathbb{G}})$, and moreover $\mathcal{F}$ sends $L^{1}(\mathbb{G})$ injectively into $c_{0}(\hat{\mathbb{G}})$.

Proof. For $\varphi \in L^{\infty}(\mathbb{G})^{*}$, recall that $\|\varphi \otimes \iota\|=\|\varphi\|$, so we have

$$
\|\hat{\varphi}\|=\sup _{\pi \in \operatorname{Irr}(\mathbb{G})}\|\hat{\varphi}(\pi)\|=\sup _{\pi \in \operatorname{Irr}(\mathbb{G})}\left\|(\varphi \otimes \iota)\left(\left(u^{(\pi)}\right)^{*}\right)\right\| \leq \sup _{\pi \in \operatorname{Irr}(\mathbb{G})}\|\varphi \otimes \iota\|\left\|u^{(\pi)}\right\|=\|\varphi\| .
$$

So $\mathcal{F}$ is a contraction. Recall that $\operatorname{Pol}(\mathbb{G})$ is dense in $L^{1}(\mathbb{G})=L^{\infty}(\mathbb{G})_{*}$ and note that $\mathcal{F}(\operatorname{Pol}(\mathbb{G})) \subset$ $c_{c}(\hat{\mathbb{G}})$, so $\mathcal{F}\left(L^{1}(\mathbb{G})\right) \subset \overline{\mathcal{F}(\operatorname{Pol}(\mathbb{G}))} \subset \overline{c_{c}(\hat{\mathbb{G}})}=c_{0}(\hat{\mathbb{G}})$. The injectivity of $\mathcal{F}$ follows from the ultraweak density of $\operatorname{Pol}(\mathbb{G})$ in $L^{\infty}(\mathbb{G})$. 
It is easy to establish the Fourier inversion formula and the Plancherel theorem for $L^{2}(\mathbb{G})$. The following result can be found in Proposition 2.6 in [Wan16].

Proposition 2.2. (a) For all $x \in L^{2}(\mathbb{G})$, we have

$$
x=\sum_{\pi \in \operatorname{Irr}(\mathbb{G})} d_{\pi}(\iota \otimes \operatorname{Tr})\left[\left(1 \otimes \hat{x}(\pi) Q_{\pi}\right) u^{(\pi)}\right],
$$

where the convergence of the series is in the $L^{2}$-sense. For any $\pi \in \operatorname{Irr}(\mathbb{G})$, if we denote by $\mathcal{E}_{\pi}$ the orthogonal projection of $L^{2}(\mathbb{G})$ onto the subspace spanned by the matrix coefficients $\left(u_{i j}^{(\pi)}\right)_{i, j=1}^{n_{\pi}}$, and write $\mathcal{E}_{\pi} x=\sum_{i, j} x_{i j}^{(\pi)} u_{i j}^{(\pi)}$ with $x_{i j}^{(\pi)} \in \mathbb{C}, X_{\pi}=\left[x_{j i}^{(\pi)}\right]_{i, j}$, then

$$
\hat{x}(\pi)=d_{\pi}^{-1} X_{\pi} Q_{\pi}^{-1} .
$$

(b) $\mathcal{F}$ is a unitary operator from $L^{2}(\mathbb{G})$ onto $\ell^{2}(\hat{\mathbb{G}})$.

For $a=\left(a_{\pi}\right)_{\pi} \in \prod_{\pi} B\left(H_{\pi}\right)$, we define the left and right multipliers $m_{a}^{L}: \operatorname{Pol}(\mathbb{G}) \rightarrow \operatorname{Pol}(\mathbb{G}), m_{a}^{R}:$ $\operatorname{Pol}(\mathbb{G}) \rightarrow \operatorname{Pol}(\mathbb{G})$ associated to $a(\mathrm{cf}$. [JNR09, Daw12]) by

$$
\left(m_{a}^{L} \otimes \iota\right) u^{(\pi)}=\left(1 \otimes a_{\pi}\right) u^{(\pi)}, \quad\left(m_{a}^{R} \otimes \iota\right) u^{(\pi)}=u^{(\pi)}\left(1 \otimes a_{\pi}\right),
$$

which, according to the above proposition, yields that

$$
\left(m_{a}^{L} x\right)^{\wedge}(\pi) Q_{\pi}=\hat{x}(\pi) Q_{\pi} a_{\pi}, \quad\left(m_{a}^{R} x\right)^{\wedge}(\pi) Q_{\pi}=a_{\pi} \hat{x}(\pi) Q_{\pi} \quad \pi \in \operatorname{Irr}(\mathbb{G}) .
$$

Denote $Q=\left(Q_{\pi}\right)_{\pi} \in \prod_{\pi} B\left(H_{\pi}\right)$ and let $1 \leq p \leq \infty$. We say that $a$ is a bounded left (resp., right) multiplier on $L^{p}(\mathbb{G})$ if $m_{a}^{L}$ (resp., $m_{a}^{R}$ ) extends to a bounded map on $L^{p}(\mathbb{G})$, and denote the set of all such multipliers by $\mathrm{M}_{L}\left(L^{p}(\mathbb{G})\right)$ (resp., $\left.\mathrm{M}_{R}\left(L^{p}(\mathbb{G})\right)\right)$. We define

$$
\mathrm{M}\left(L^{p}(\mathbb{G})\right)=\left\{a \in \prod_{\pi} B\left(H_{\pi}\right): Q^{-1 / p} a Q^{1 / p} \in \mathrm{M}_{L}\left(L^{p}(\mathbb{G})\right), a \in \mathrm{M}_{R}\left(L^{p}(\mathbb{G})\right)\right\}
$$

equipped with the norm

$$
\|a\|_{\mathrm{M}\left(L^{p}(\mathbb{G})\right)}=\max \left\{\left\|m_{Q^{-1 / p} a Q^{1 / p}}^{L}\right\|_{B\left(L^{p}(\mathbb{G})\right)},\left\|m_{a}^{R}\right\|_{B\left(L^{p}(\mathbb{G})\right)}\right\} .
$$

Lemma 2.3. For any $a \in \prod_{\pi} B\left(H_{\pi}\right)$, we have

$$
\|a\|_{\infty}=\left\|m_{Q^{-1 / 2} a Q^{1 / 2}}^{L}\right\|_{B\left(L^{2}(\mathbb{G})\right)}=\left\|m_{a}^{R}\right\|_{B\left(L^{2}(\mathbb{G})\right)} .
$$

In particular, we have the following isometric isomorphism

$$
\mathrm{M}\left(L^{2}(\mathbb{G})\right)=\ell^{\infty}(\hat{\mathbb{G}}) .
$$

Proof. Let $a \in \ell^{\infty}(\hat{\mathbb{G}})$. By equality (2.8) and Proposition 2.2, we have for all $x \in \operatorname{Pol}(\mathbb{G})$,

$$
\begin{aligned}
\left\|m_{Q^{-1 / 2} a Q^{1 / 2}}^{L} x\right\|_{2}^{2} & =\sum_{\pi \in \operatorname{Irr}(\mathbb{G})} d_{\pi} \operatorname{Tr}\left(\left|\hat{x}(\pi) Q_{\pi}^{1 / 2} a_{\pi} Q_{\pi}^{-1 / 2}\right|^{2} Q_{\pi}\right) \\
& =\sum_{\pi \in \operatorname{Irr}(\mathbb{G})} d_{\pi} \operatorname{Tr}\left(\hat{x}(\pi) Q_{\pi}^{1 / 2} a_{\pi} a_{\pi}^{*} Q_{\pi}^{1 / 2} \hat{x}(\pi)^{*}\right) \\
& \leq\|a\|_{\infty}^{2} \sum_{\pi \in \operatorname{Irr}(\mathbb{G})} d_{\pi} \operatorname{Tr}\left(\hat{x}(\pi) Q_{\pi} \hat{x}(\pi)^{*}\right)=\|a\|_{\infty}^{2}\|x\|_{2}^{2} .
\end{aligned}
$$

Therefore we get

$$
Q^{-1 / 2} a Q^{1 / 2} \in \mathrm{M}_{L}\left(L^{2}(\mathbb{G})\right), \quad\left\|m_{Q^{-1 / 2} a Q^{1 / 2}}^{L}\right\|_{B\left(L^{2}(\mathbb{G})\right)} \leq\|a\|_{\infty} .
$$

Conversely, take $a \in \prod_{\pi} B\left(H_{\pi}\right)$ with $Q^{-1 / 2} a Q^{1 / 2} \in \mathrm{M}_{L}\left(L^{2}(\mathbb{G})\right)$. For $\pi \in \operatorname{Irr}(\mathbb{G})$ and for each eigenvalue $\lambda$ of the operator $a_{\pi} a_{\pi}^{*}$, we choose an orthogonal projection $E \in B\left(H_{\pi}\right)$ such that $a_{\pi} a_{\pi}^{*} E=\lambda E$. According to Proposition 2.2, we choose an $x \in \operatorname{Pol}(\mathbb{G})$ with

$$
\hat{x}(\pi)=E Q_{\pi}^{-1 / 2}, \quad \hat{x}\left(\pi^{\prime}\right)=0, \quad \pi^{\prime} \neq \pi .
$$


Then we have

$$
\begin{aligned}
\left\|m_{Q^{-1 / 2} a Q^{1 / 2}}^{L} x\right\|_{2}^{2} & =d_{\pi} \operatorname{Tr}\left(\left|\hat{x}(\pi) Q_{\pi}^{1 / 2} a_{\pi} Q_{\pi}^{-1 / 2}\right|^{2} Q_{\pi}\right) \\
& =d_{\pi} \operatorname{Tr}\left(a_{\pi}^{*} Q_{\pi}^{1 / 2} \hat{x}(\pi)^{*} \hat{x}(\pi) Q_{\pi}^{1 / 2} a_{\pi}\right)=d_{\pi} \operatorname{Tr}\left(a_{\pi}^{*} E a_{\pi}\right)=\lambda d_{\pi} \operatorname{Tr}(E) \\
& =\lambda d_{\pi} \operatorname{Tr}\left(E^{*} E\right)=\lambda d_{\pi} \operatorname{Tr}\left(Q_{\pi}^{1 / 2} \hat{x}(\pi)^{*} \hat{x}(\pi) Q_{\pi}^{1 / 2}\right)=\lambda\|x\|_{2}^{2} .
\end{aligned}
$$

So we see that $\lambda \leq\left\|m_{Q^{-1 / 2} a Q^{1 / 2}}^{L}\right\|_{B\left(L^{2}(\mathbb{G})\right)}^{2}$. Taking the supremum over all such eigenvalues $\lambda$, we get

$$
a \in \ell^{\infty}(\hat{\mathbb{G}}), \quad\|a\|_{\infty} \leq\left\|m_{Q^{-1 / 2} a Q^{1 / 2}}^{L}\right\|_{B\left(L^{2}(\mathbb{G})\right)} .
$$

Combining the two inequalities (2.9) and (2.10) we prove that for all $a \in \prod_{\pi} B\left(H_{\pi}\right)$ we have

$$
\|a\|_{\infty}=\left\|m_{Q^{-1 / 2} a Q^{1 / 2}}^{L}\right\|_{B\left(L^{2}(\mathbb{G})\right)} .
$$

A similar argument gives

So we establish the lemma.

$$
\|a\|_{\infty}=\left\|m_{a}^{R}\right\|_{B\left(L^{2}(\mathbb{G})\right)}
$$

It is well known that if $\mathbb{G}$ is a compact group $G$ or the dual compact quantum group $\hat{\Gamma}$ of a discrete group $\Gamma$, then for all $1 \leq p \leq \infty$ we have

$$
\|a\|_{\infty} \leq\|a\|_{\mathrm{M}\left(L^{p}(\mathbb{G})\right)}, \quad a \in \mathrm{M}\left(L^{p}(\mathbb{G})\right) .
$$

We refer to [Har99, HR70] for related discussions. This is however not clear for an arbitrary compact quantum group. Note that if the operators $m_{a}^{L}$ and $m_{a}^{R}$ are completely bounded on $L^{\infty}(\mathbb{G})$, then from $(2.7)$ we see that

$$
\|a\|_{\infty}=\left\|\left(\left(1 \otimes a_{\pi}\right) u^{(\pi)}\right)\right\|_{\oplus\left(C(\mathbb{G}) \otimes B\left(H_{\pi}\right)\right)} \leq\left\|m_{a}^{L}\right\|_{C B\left(L^{\infty}(\mathbb{G})\right)}, \quad\|a\|_{\infty} \leq\left\|m_{a}^{R}\right\|_{C B\left(L^{\infty}(\mathbb{G})\right)},
$$

where \|\|$_{C B\left(L^{\infty}(\mathbb{G})\right)}$ denote the completely bounded norm in the sense of [ER00]. If the multiplier $a \in \mathrm{M}\left(L^{\infty}(\mathbb{G})\right)$ is not necessarily completely bounded, it is even not clear to see whether the sequence $a \in \prod_{\pi} B\left(H_{\pi}\right)$ is bounded or not. We refer to [Daw10, Section 8.2] for some related discussions. In the following Proposition 2.7 we will give an affirmative answer in the case that $\mathbb{G}$ is of Kac type, and indeed give a similar estimate for the general case, which also improves an early result in [Daw10, Proposition 8.8] for compact quantum groups. Let us first establish some easy but useful lemmas.

Lemma 2.4. Let $1 \leq p \leq \infty$. We have the following assertions:

(a) For all $t \in \mathbb{R}$, the operator $\sigma_{t}$ extends to an isometry on $L^{p}(\mathbb{G})$.

(b) We have $\sigma_{-\mathrm{i} / p} \circ R=R \circ \sigma_{\mathrm{i} / p}$, and they extend to an isometry on $L^{p}(\mathbb{G})$.

(c) The map $x \mapsto \sigma_{-\mathrm{i} / p}\left(x^{*}\right)$ extends to an isometry on $L^{p}(\mathbb{G})$.

Proof. (a) Since $h \circ \sigma=h$, it is easy to see that the operators $\sigma_{t}$ and $\sigma_{-t}$ extend to isometries on $L^{1}(\mathbb{G})$ and $L^{\infty}(\mathbb{G})$ for all $t \in \mathbb{R}$. So by interpolation $\sigma_{t}$ also extends to an isometry on $L^{p}(\mathbb{G})$ for all $t \in \mathbb{R}$.

(b) It is well known and easy to see from (1.3), (1.4) and (1.5) that

$$
S \circ \sigma_{z}=\sigma_{-z} \circ S, \quad R \circ \sigma_{z}=\sigma_{-z} \circ R, \quad z \in \mathbb{C} .
$$

Also recall that $R$ is a $*$-antiautomorphism on $L^{\infty}(\mathbb{G})$ with $h \circ R=h$, and $h(x y)=h\left(y \sigma_{-\mathrm{i}}(x)\right)$ for $x, y \in \operatorname{Pol}(\mathbb{G})$. So for $x \in \operatorname{Pol}(\mathbb{G})$, we have

$$
\begin{aligned}
\|x\|_{1} & =\sup _{y \in \operatorname{Pol}(\mathbb{G}),\|y\|_{\infty}=1}|h(y x)|=\sup _{y \in \operatorname{Pol}(\mathbb{G}),\|y\|_{\infty}=1}|h(R(x) R(y))| \\
& =\sup _{y \in \operatorname{Pol}(\mathbb{G}),\|y\|_{\infty}=1}\left|h\left(R(y) \sigma_{-\mathrm{i}}(R(x))\right)\right|=\left\|\sigma_{-\mathrm{i}}(R(x))\right\|_{1} .
\end{aligned}
$$

Thus we see that $\sigma_{-\mathrm{i}} \circ R$ extends to an isometry on $L^{1}(\mathbb{G})$. Therefore by (a), $\sigma_{-\mathrm{i}-t} \circ R$ and $R \circ \sigma_{\mathrm{i}+t}$ also extend to isometries on $L^{1}(\mathbb{G})$ for $t \in \mathbb{R}$. Then the assertion follows directly from the Stein interpolation theorem (see e.g. [Lun09, Theorem 2.7]).

(c) This follows directly from Proposition 1.5 (1)(4). 
Lemma 2.5. Let $1 \leq p \leq \infty$. Consider $\mathbf{E} \subset \operatorname{Irr}(\mathbb{G})$ and

$$
X=\operatorname{span}\left\{u_{i j}^{(\pi)},\left(u_{i j}^{(\pi)}\right)^{*}: \pi \in \mathbf{E}, 1 \leq i, j \leq n_{\pi}\right\} .
$$

We equip the space $X$ with the norm \|\|$_{p}$. Then we have for all $a \in \prod_{\pi} B\left(H_{\pi}\right)$,

$$
\left\|\left.m_{a}^{R}\right|_{X}\right\|_{B(X)}=\left\|\left.m_{Q^{-1 / 2} a^{*} Q^{1 / 2}}^{L}\right|_{X}\right\|_{B(X)} .
$$

Proof. Note that the subspace $X$ is invariant under $R, \sigma$ and $\tau$, and that the maps $m_{a}^{R}$ and $m_{Q^{-1 / 2} a^{*} Q^{1 / 2}}^{L}$ send $X$ into $X$ itself. We define a linear functional $\varphi$ on $\operatorname{Pol}(\mathbb{G})$ by $\varphi\left(u_{i j}^{(\pi)}\right)=\overline{\left(a_{\pi}\right)_{j i}}$ for $\pi \in \operatorname{Irr}(\mathbb{G})$ and $1 \leq i, j \leq n_{\pi}$. Then we see that $\widehat{\varphi^{*}}=a$ where $\varphi^{*}=\overline{\varphi(\cdot *)}$. Take $x \in X$. By (2.4), (2.5), we have

$$
m_{a}^{R}\left(\sigma_{-\mathrm{i} / p}\left(x^{*}\right)\right)=\left(\iota \otimes\left(\varphi^{*} \circ S\right)\right) \Delta\left(\sigma_{-\mathrm{i} / p}\left(x^{*}\right)\right) .
$$

Recall from (1.4) and (1.6) that

$$
S=\tau_{-\mathrm{i} / 2} \circ R, \quad R^{2}=\mathrm{id}, \quad \Sigma \circ \Delta \circ R=(R \otimes R) \circ \Delta .
$$

So we get

$$
\begin{aligned}
\left(\iota \otimes\left(\varphi^{*} \circ S\right)\right) \Delta\left(\sigma_{-\mathrm{i} / p}\left(x^{*}\right)\right) & =\left(R \otimes\left(\varphi^{*} \circ \tau_{-\mathrm{i} / 2}\right)\right)(R \otimes R) \Delta\left(\sigma_{-\mathrm{i} / p}\left(x^{*}\right)\right) \\
& =\left(\left(\varphi^{*} \circ \tau_{-\mathrm{i} / 2}\right) \otimes R\right) \Delta\left(R \circ \sigma_{-\mathrm{i} / p}\left(x^{*}\right)\right) .
\end{aligned}
$$

Writing $R=\left(R \circ \sigma_{\mathrm{i} / p}\right) \circ \sigma_{-\mathrm{i} / p}$ and using the previous lemma, we then have

$$
\begin{aligned}
\left\|m_{a}^{R}\left(\sigma_{-\mathrm{i} / p}\left(x^{*}\right)\right)\right\|_{p} & =\left\|\sigma_{-\mathrm{i} / p}\left(\left(\varphi^{*} \circ \tau_{-\mathrm{i} / 2}\right) \otimes \iota\right) \Delta\left(R \circ \sigma_{-\mathrm{i} / p}\left(x^{*}\right)\right)\right\|_{p} \\
& =\left\|\sigma_{-\mathrm{i} / p}\left(\left[\left(\left(\varphi \circ \tau_{\mathrm{i} / 2}\right) \otimes \iota\right) \Delta\left(R \circ \sigma_{\mathrm{i} / p}(x)\right)\right]^{*}\right)\right\|_{p} \\
& =\left\|\left(\left(\varphi \circ \tau_{\mathrm{i} / 2}\right) \otimes \iota\right) \Delta\left(R \circ \sigma_{\mathrm{i} / p}(x)\right)\right\|_{p} .
\end{aligned}
$$

Note that by (2.1) and (1.5), we have

$$
\left(\varphi \circ \tau_{\mathrm{i} / 2} \circ S^{-1}\right)^{\wedge}=Q^{-1 / 2} a^{*} Q^{1 / 2} .
$$

So together with (2.5) and (2.8), we have

$$
\left(\left(\varphi \circ \tau_{\mathrm{i} / 2}\right) \otimes \iota\right) \Delta\left(R \circ \sigma_{\mathrm{i} / p}(x)\right)=m_{Q^{-1 / 2} a^{*} Q^{1 / 2}}^{L}\left(R \circ \sigma_{-\mathrm{i} / p}(x)\right)
$$

and hence the above computations yield

$$
\left\|m_{a}^{R}\left(\sigma_{-\mathrm{i} / p}\left(x^{*}\right)\right)\right\|_{p}=\left\|m_{Q^{-1 / 2} a^{*} Q^{1 / 2}}^{L}\left(R \circ \sigma_{\mathrm{i} / p}(x)\right)\right\|_{p} .
$$

Since the maps $x \mapsto \sigma_{-\mathrm{i} / p}\left(x^{*}\right)$ and $x \mapsto R \circ \sigma_{\mathrm{i} / p}(x)$ are isometries on $X$ according to the previous lemma, we obtain the desired conclusion.

Lemma 2.6. For $z \in \mathbb{C}$ and $x \in \operatorname{Pol}(\mathbb{G})$, we have

$$
\mathcal{F}\left(\sigma_{z}(x)\right)=Q^{\mathrm{i} z} \hat{x} Q^{\mathrm{i} z} .
$$

In particular for $a \in \prod_{\pi \in \operatorname{Irr}(\mathbb{G})} B\left(H_{\pi}\right)$ and $t \in \mathbb{R}$,

$$
\sigma_{t} \circ m_{a}^{L} \circ \sigma_{-t}=m_{Q^{-\mathrm{i} t} a Q^{\mathrm{i} t}}^{L}, \quad \sigma_{t} \circ m_{a}^{R} \circ \sigma_{-t}=m_{Q^{\mathrm{it}} a Q^{-\mathrm{i} t}}^{R} .
$$

Proof. Recall that

$$
\sigma_{z}(x)=\left(f_{\mathrm{i} z} \otimes \iota \otimes f_{\mathrm{i} z}\right) \Delta^{(2)}(x), \quad x \in \operatorname{Pol}(\mathbb{G}), z \in \mathbb{C} .
$$

And by the construction of $\left(f_{z}\right)$ and $(2.1)$ we have $\left(f_{\mathrm{i} z} \circ S^{-1}\right)^{\wedge}=Q^{\mathrm{i} z}$. So together with (2.4) and (2.5) we get

Consequently, for $x \in \operatorname{Pol}(\mathbb{G})$,

$$
\mathcal{F}\left(\sigma_{z}(x)\right)=Q^{\mathrm{i} z} \hat{x} Q^{\mathrm{i} z}
$$

$$
\mathcal{F}\left(\sigma_{t} \circ m_{a}^{L} \circ \sigma_{-t}(x)\right) Q=Q^{\mathrm{i} t} \mathcal{F}\left(m_{a}^{L} \circ \sigma_{-t}(x)\right) Q^{1+\mathrm{i} t}=Q^{\mathrm{i} t} \mathcal{F}\left(\sigma_{-t}(x)\right) Q a Q^{\mathrm{i} t}=\hat{x} Q Q^{\mathrm{i} t} a Q^{-\mathrm{i} t} .
$$

That is,

as desired. The equality for $m_{a}^{R}$ follows similarly.

$$
\sigma_{t} \circ m_{a}^{L} \circ \sigma_{-t}=m_{Q^{-\mathrm{i} t} a Q^{\mathrm{i} t}}^{L},
$$


Now we are able to give an analogue of inequality (2.11) for general compact quantum groups. The proof below is based on a personal communication by Marius Junge.

Proposition 2.7. Let $1 \leq p \leq \infty$. For all $a \in \prod_{\pi} B\left(H_{\pi}\right)$ and all $0 \leq \theta \leq 1$, we have

$$
\left\|Q^{\frac{1}{4}-\frac{\theta}{2}} a Q^{-\frac{1}{4}+\frac{\theta}{2}}\right\|_{\infty} \leq\left\|m_{a}^{R}\right\|_{B\left(L^{p}(\mathbb{G})\right)}^{1 / 2}\left\|m_{Q^{-\theta} a Q^{\theta}}^{L}\right\|_{B\left(L^{p}(\mathbb{G})\right)}^{1 / 2} .
$$

Proof. Assume that $m_{a}^{R}$ is bounded on $L^{p}(\mathbb{G})$. Consider the map

$$
T: \operatorname{Pol}(\mathbb{G}) \rightarrow \operatorname{Pol}(\mathbb{G}), \quad y \mapsto \sigma_{-\mathrm{i} / p}\left(\left[m_{a}^{R}\left(\sigma_{-\mathrm{i} / p}\left(y^{*}\right)\right)\right]^{*}\right) .
$$

By Lemma 2.4, $T$ extends to a bounded operator on $L^{p}(\mathbb{G})$ with $\|T\|_{B\left(L^{p}(\mathbb{G})\right)}=\left\|m_{a}^{R}\right\|_{B\left(L^{p}(\mathbb{G})\right)}$. We let $1 \leq p^{\prime} \leq \infty$ with $1 / p+1 / p^{\prime}=1$ and denote $\langle\cdot, \cdot\rangle_{p^{\prime}, p}$ the duality bracket between $L^{p^{\prime}}(\mathbb{G})$ and $L^{p}(\mathbb{G})$ defined by

$$
\langle x, y\rangle_{p^{\prime}, p}=\operatorname{tr}\left(x D^{1 / p^{\prime}} y D^{1 / p}\right), \quad x, y \in \operatorname{Pol}(\mathbb{G}) .
$$

First let $1 \leq p<\infty$. Then consider the adjoint map $T^{*}: L^{p}(\mathbb{G})^{*} \rightarrow L^{p}(\mathbb{G})^{*}$ of $T$ and recall the formula $\sigma_{t}(x)=D^{\mathrm{i} t} x D^{-\mathrm{i} t}$ in Proposition 1.5. We have for $x, y \in \operatorname{Pol}(\mathbb{G})$,

$$
\begin{aligned}
\left\langle T^{*} x, y\right\rangle_{p^{\prime}, p} & =\langle x, T y\rangle_{p^{\prime}, p}=\operatorname{tr}\left(x D^{1 / p^{\prime}} T y D^{1 / p}\right)=\operatorname{tr}\left(x D\left[m_{a}^{R}\left(\sigma_{-\mathrm{i} / p}\left(y^{*}\right)\right)\right]^{*}\right) \\
& =h\left(\left[m_{a}^{R}\left(\sigma_{-\mathrm{i} / p}\left(y^{*}\right)\right)\right]^{*} x\right) .
\end{aligned}
$$

According to Proposition 2.2 and (2.8), we note that

$$
\begin{aligned}
h\left(\left[m_{a}^{R}\left(\sigma_{-\mathrm{i} / p}\left(y^{*}\right)\right)\right]^{*} x\right) & =\hat{h}\left(\left(\left[m_{a}^{R}\left(\sigma_{-\mathrm{i} / p}\left(y^{*}\right)\right)\right]^{\wedge}\right)^{*} \hat{x}\right)=\hat{h}\left(\left(\left[a\left(\sigma_{-\mathrm{i} / p}\left(y^{*}\right)\right)^{\wedge}\right]\right)^{*} \hat{x}\right) \\
& =\hat{h}\left(\left[\left(\sigma_{-\mathrm{i} / p}\left(y^{*}\right)\right)^{\wedge}\right]^{*} a^{*} \hat{x}\right)=h\left(\left(\sigma_{-\mathrm{i} / p}\left(y^{*}\right)\right)^{*} m_{a^{*}}^{R} x\right) .
\end{aligned}
$$

Observe that $\left(\sigma_{-\mathrm{i} / p}\left(y^{*}\right)\right)^{*}=D^{-1 / p} y D^{1 / p}$. Therefore the above equalities give

$$
\left\langle T^{*} x, y\right\rangle_{p^{\prime}, p}=h\left(\left(\sigma_{-\mathrm{i} / p}\left(y^{*}\right)\right)^{*} m_{a^{*}}^{R} x\right)=\operatorname{tr}\left(m_{a^{*}}^{R} x D^{1 / p^{\prime}} y D^{1 / p}\right)=\left\langle m_{a^{*}}^{R} x, y\right\rangle_{p^{\prime}, p} .
$$

So $m_{a^{*}}^{R}$ extends to an isometry on $L^{p^{\prime}}(\mathbb{G})$ with

$$
\left\|m_{a^{*}}^{R}\right\|_{B\left(L^{p^{\prime}}(\mathbb{G})\right)}=\|T\|_{B\left(L^{p}(\mathbb{G})\right)}=\left\|m_{a}^{R}\right\|_{B\left(L^{p}(\mathbb{G})\right)}
$$

If $p=\infty$, we consider the restriction of operator $\left.T^{*}\right|_{L^{1}(\mathbb{G})}: L^{1}(\mathbb{G}) \rightarrow L^{1}(\mathbb{G})$ instead of $T^{*}$ : $L^{\infty}(\mathbb{G})^{*} \rightarrow L^{\infty}(\mathbb{G})^{*}$, and repeat the above argument. Then $(2.12)$ also holds for $p=\infty$.

On the other hand, we assume that $m_{Q^{-\theta} a Q^{\theta}}^{L}$ is bounded on $L^{p}(\mathbb{G})$. By Lemma 2.5, we have

$$
\left\|m_{Q^{-\frac{1}{2}+\theta} a^{*} Q^{\frac{1}{2}-\theta}}^{R}\right\|_{B\left(L^{p}(\mathbb{G})\right)}=\left\|m_{Q^{-\theta} a Q^{\theta}}^{L}\right\|_{B\left(L^{p}(\mathbb{G})\right)} .
$$

Note also that by Lemma 2.4 and Lemma 2.6 , for all $t \in \mathbb{R}$,

$$
\begin{aligned}
\left\|m_{Q^{\left(-\frac{1}{2}+\theta\right)(1+\mathrm{i} t)} a^{*} Q^{\left(\frac{1}{2}-\theta\right)(1+\mathrm{i} t)}}^{R}\right\|_{B\left(L^{p}(\mathbb{G})\right)} & =\left\|\sigma_{\left(-\frac{1}{2}+\theta\right) t} \circ m_{Q^{-\frac{1}{2}+\theta} a^{*} Q^{\frac{1}{2}-\theta}}^{R} \circ \sigma_{\left(\frac{1}{2}-\theta\right) t}\right\|_{B\left(L^{p}(\mathbb{G})\right)} \\
& =\left\|m_{Q^{-\frac{1}{2}+\theta} a^{*} Q^{\frac{1}{2}-\theta}}^{R}\right\|_{B\left(L^{p}(\mathbb{G})\right)} .
\end{aligned}
$$

Hence, applying the Stein interpolation theorem, (2.12) and (2.13) yields

$$
\begin{aligned}
\left\|m_{Q^{-\frac{1}{4}+\frac{\theta}{2}} a^{*} Q^{\frac{1}{4}-\frac{\theta}{2}}}^{R}\right\|_{B\left(L^{2}(\mathbb{G})\right)} & \leq\left\|m_{a^{*}}^{R}\right\|_{B\left(L^{p^{\prime}}(\mathbb{G})\right)}^{\frac{1}{2}}\left\|m_{Q^{-\frac{1}{2}+\theta} a^{*} Q^{\frac{1}{2}-\theta}}^{R}\right\|_{B\left(L^{p}(\mathbb{G})\right)}^{\frac{1}{2}} \\
& =\left\|m_{a}^{R}\right\|_{B\left(L^{p}(\mathbb{G})\right)}^{\frac{1}{2}}\left\|m_{Q^{-\theta} a Q^{\theta}}^{L}\right\|_{B\left(L^{p}(\mathbb{G})\right)}^{\frac{1}{2}}
\end{aligned}
$$

Now applying Lemma 2.3, we get

$$
\left\|Q^{\frac{1}{4}-\frac{\theta}{2}} a Q^{-\frac{1}{4}+\frac{\theta}{2}}\right\|_{\infty} \leq\left\|m_{a}^{R}\right\|_{B\left(L^{p}(\mathbb{G})\right)}^{\frac{1}{2}}\left\|m_{Q^{-\theta} a Q^{\theta}}^{L}\right\|_{B\left(L^{p}(\mathbb{G})\right)}^{\frac{1}{2}}
$$

as desired.

In particular, we may state the following corollary.

Corollary 2.8. Assume that $\mathbb{G}$ is of Kac type. Let $1 \leq p \leq \infty$. For all $a \in \prod_{\pi} B\left(H_{\pi}\right)$, we have

$$
\|a\|_{\infty} \leq\|a\|_{\mathrm{M}\left(L^{p}(\mathbb{G})\right)} .
$$


In the following paragraph we present a special subspace of $C_{r}(\mathbb{G})$, which, in the classical case, is known as the Fourier algebra introduced by Eymard [Eym64], as explained in [HR70, p.367]. Recall that we always identify $C_{r}(\mathbb{G}) \subset L^{\infty}(\mathbb{G})$ as a subspace of $L^{1}(\mathbb{G})$.

Proposition 2.9. Let $A(\mathbb{G})=\left\{x \in L^{1}(\mathbb{G}): \hat{x} \in \ell^{1}(\hat{\mathbb{G}})\right\}$. Then $A(\mathbb{G}) \subset C_{r}(\mathbb{G})$ and

$$
\|x\|_{\infty} \leq\|\hat{x}\|_{1}, \quad x \in A(\mathbb{G}) .
$$

Moreover, if we let $\|x\|_{A}=\|\hat{x}\|_{1}$ for $x \in A(\mathbb{G})$, then $\left(A(\mathbb{G}),\|\cdot\|_{A}\right)$ is a Banach space isometrically isomorphic to $\ell^{1}(\hat{\mathbb{G}})$.

Proof. Firstly we show that

$$
\|x\|_{\infty} \leq\|\hat{x}\|_{1}, \quad x \in \operatorname{Pol}(\mathbb{G}) .
$$

Choose an $x \in \operatorname{Pol}(\mathbb{G})$. For each $\pi \in \operatorname{Irr}(\mathbb{G})$, by the identification $L^{\infty}(\mathbb{G})=\left(L^{\infty}(\mathbb{G})_{*}\right)^{*}$ and the Hölder inequality on $\operatorname{Tr}$ we have

$$
\begin{aligned}
\left\|(\iota \otimes \operatorname{Tr})\left[\left(1 \otimes \hat{x}(\pi) Q_{\pi}\right) u^{(\pi)}\right]\right\|_{\infty} & =\sup _{\omega \in L^{\infty}(\mathbb{G})_{*},\|\omega\|=1}\left|\omega\left((\iota \otimes \operatorname{Tr})\left[\left(1 \otimes \hat{x}(\pi) Q_{\pi}\right) u^{(\pi)}\right]\right)\right| \\
& =\sup _{\omega \in L^{\infty}(\mathbb{G})_{*},\|\omega\|=1}\left|\operatorname{Tr}\left(\hat{x}(\pi) Q_{\pi}\left[(\omega \otimes \iota) u^{(\pi)}\right]\right)\right| \\
& \leq \sup _{\omega \in L^{\infty}(\mathbb{G})_{*},\|\omega\|=1} \operatorname{Tr}\left(\left|\hat{x}(\pi) Q_{\pi}\right|\right)\left\|(\omega \otimes \iota) u^{(\pi)}\right\| \\
& \leq \operatorname{Tr}\left(\left|\hat{x}(\pi) Q_{\pi}\right|\right) .
\end{aligned}
$$

Therefore by (2.6),

$$
\|x\|_{\infty} \leq \sum_{\pi \in \operatorname{Irr}(\mathbb{G})} d_{\pi}\left\|(\iota \otimes \operatorname{Tr})\left[\left(1 \otimes \hat{x}(\pi) Q_{\pi}\right) u^{(\pi)}\right]\right\|_{\infty} \leq \sum_{\pi \in \operatorname{Irr}(\mathbb{G})} d_{\pi} \operatorname{Tr}\left(\left|\hat{x}(\pi) Q_{\pi}\right|\right)=\|\hat{x}\|_{1} .
$$

Now given $y \in A(\mathbb{G})$, we note that $c_{c}(\hat{\mathbb{G}})$ is dense in $\ell^{1}(\hat{\mathbb{G}})$ and $\mathcal{F}(\operatorname{Pol}(\mathbb{G}))=c_{c}(\hat{\mathbb{G}})$, so we may take $x_{n} \in \operatorname{Pol}(\mathbb{G})$ such that $\left\|\hat{x}_{n}-\hat{y}\right\|_{1} \rightarrow 0$. So $\left(\hat{x}_{n}\right)_{n}$ is $\|\cdot\|_{1}$-Cauchy, and hence by $(2.15)$ the sequence $\left(x_{n}\right)_{n}$ is $\|\cdot\|_{\infty}$-Cauchy in $\operatorname{Pol}(\mathbb{G})$. Since $\operatorname{Pol}(\mathbb{G})$ is dense in $C_{r}(\mathbb{G})$, the sequence $\left(x_{n}\right)_{n}$ in $\operatorname{Pol}(\mathbb{G})$ converges to some $x \in C_{r}(\mathbb{G})$. Note that $\|\cdot\|_{1} \leq\|\cdot\|_{\infty}$ on $L^{\infty}(\mathbb{G})$, we have also $\left\|x_{n}-x\right\|_{1} \rightarrow 0$. Then according to the contractive property of $\overline{\mathcal{F}}$, it holds that $\hat{x}(\pi)=\lim _{n} \hat{x}_{n}(\pi)=\hat{y}(\pi)$ for all $\pi \in \operatorname{Irr}(\mathbb{G})$. So $y=x \in C_{r}(\mathbb{G})$ according to the injectivity of $\mathcal{F}$, and $\|y\|_{\infty}=\lim _{n}\left\|x_{n}\right\|_{\infty} \leq$ $\lim _{n}\left\|\hat{x}_{n}\right\|_{1}=\|\hat{y}\|_{1}$. As a result $A(\mathbb{G}) \subset C_{r}(\mathbb{G})$ and $(2.14)$ is proved.

Now we set $\|x\|_{A}=\|\hat{x}\|_{1}$ for all $x \in A(\mathbb{G})$, then $\left(A(\mathbb{G}),\|\cdot\|_{A}\right)$ is clearly a normed space and the map $x \mapsto \hat{x}$ sends $\left(A(\mathbb{G}),\|\cdot\|_{A}\right)$ isometrically into $\ell^{1}(\hat{\mathbb{G}})$. To see that it is surjective, we shall show that for any $a=\left(a_{\pi}\right)_{\pi \in \operatorname{Irr}(\mathbb{G})} \in \ell^{1}(\hat{\mathbb{G}})$ there exists $x \in L^{1}(\mathbb{G})$ with $\hat{x}=a$. In fact, if we consider a sequence $x_{n} \in \operatorname{Pol}(\mathbb{G})$ such that $\left\|\hat{x}_{n}-a\right\|_{1} \rightarrow 0$, then the existence of $x \in L^{1}(\mathbb{G})$ with $\hat{x}=a$ follows simply from the same argument as in the preceding paragraph.

In some literature the Fourier algebra $A(\mathbb{G})$ is also defined simply to be the space $\ell^{1}(\hat{\mathbb{G}})$. Note that the identification that we use yields also the product on $A(\mathbb{G})$ (convolution product of $\ell^{1}(\hat{\mathbb{G}}) \cong \ell^{\infty}(\hat{\mathbb{G}})_{*}$, well-known in the theory of locally compact quantum groups), which makes $A(\mathbb{G})$ a Banach algebra.

\section{SIDON SETS}

Let $\mathbb{G}$ be a compact quantum group and $\mathbf{E}$ be a subset of $\operatorname{Irr}(\mathbb{G})$. Define

and

$$
\begin{gathered}
L_{\mathbf{E}}^{\infty}(\mathbb{G})=\left\{x \in L^{\infty}(\mathbb{G}): \hat{x}(\pi)=0 \text { if } \pi \in \operatorname{Irr}(\mathbb{G}) \backslash \mathbf{E}\right\}, \\
C_{\mathbf{E}}(\mathbb{G})=C_{r}(\mathbb{G}) \cap L_{\mathbf{E}}^{\infty}(\mathbb{G}), \quad \operatorname{Pol}_{\mathbf{E}}(\mathbb{G})=\operatorname{Pol}(\mathbb{G}) \cap L_{\mathbf{E}}^{\infty}(\mathbb{G}),
\end{gathered}
$$

$$
\begin{gathered}
\ell^{\infty}(\mathbf{E})=\left\{\left(a_{\pi}\right)_{\pi \in \operatorname{Irr}(\mathbb{G})} \in \ell^{\infty}(\hat{\mathbb{G}}): a_{\pi}=0 \text { if } \pi \in \operatorname{Irr}(\mathbb{G}) \backslash \mathbf{E}\right\}, \\
\ell^{1}(\mathbf{E})=\ell^{1}(\hat{\mathbb{G}}) \cap \ell^{\infty}(\mathbf{E}), c_{0}(\mathbf{E})=c_{0}(\hat{\mathbb{G}}) \cap \ell^{\infty}(\mathbf{E}), c_{c}(\mathbf{E})=c_{c}(\hat{\mathbb{G}}) \cap \ell^{\infty}(\mathbf{E}) .
\end{gathered}
$$


Then the subspaces $L_{\mathbf{E}}^{\infty}(\mathbb{G}), C_{\mathbf{E}}(\mathbb{G}), \ell^{\infty}(\mathbf{E}), \ell^{1}(\mathbf{E})$ are all closed subspaces. Note that we may identify the duality between spaces

$$
c_{0}(\mathbf{E})^{*}=\ell^{1}(\mathbf{E}), \quad \ell^{1}(\mathbf{E})^{*}=\ell^{\infty}(\mathbf{E})
$$

via the bracket $\langle a, b\rangle=\hat{h}(b a)=\sum_{\pi} d_{\pi} \operatorname{Tr}\left(a_{\pi} Q_{\pi} b_{\pi}\right)$ for $a \in \ell^{1}(\mathbf{E}), b \in \ell^{\infty}(\mathbf{E})$.

Definition 3.1. We say that a subset $\mathbf{E} \subset \operatorname{Irr}(\mathbb{G})$ is a Sidon set (with constant $K$ ) if there exists $K>0$ such that for any $x \in \operatorname{Pol}_{\mathbf{E}}(\mathbb{G})$, we have

$$
\|\hat{x}\|_{1} \leq K\|x\|_{\infty}
$$

Remark 3.2. Any finite subset of $\operatorname{Irr}(\mathbb{G})$ is clearly a Sidon set. Let $\mathbf{E} \subset \operatorname{Irr}(\mathbb{G})$ be a Sidon set and $\mathbf{F} \subset \operatorname{Irr}(\mathbb{G})$ be finite. Then $\mathbf{E} \cup \mathbf{F}$ is also a Sidon set. In fact, assume without loss of generality that $\mathbf{E} \cup \mathbf{F}=\emptyset$, and take $x \in \operatorname{Pol}_{\mathbf{E}}(\mathbb{G})$ and $y \in \operatorname{Pol}_{\mathbf{F}}(\mathbb{G})$. Since $\operatorname{Pol}_{\mathbf{F}}(\mathbb{G})$ is finite-dimensional, it is complemented in $L^{\infty}(\mathbb{G})$. Therefore, there exist two constants $K_{1}, K_{2}>0$ such that

$$
\begin{aligned}
\|\hat{x}+\hat{y}\|_{1} & \leq\|\hat{x}\|_{1}+\|\hat{y}\|_{1} \leq K_{1}\|x\|_{\infty}+K_{1}\|y\|_{\infty} \leq K_{1}\|x+y\|_{\infty}+2 K_{1}\|y\|_{\infty} \\
& \leq K_{1}\|x+y\|_{\infty}+2 K_{1} K_{2}\|x+y\|_{\infty}=\left(K_{1}+2 K_{1} K_{2}\right)\|x+y\|_{\infty} .
\end{aligned}
$$

We first give the following fundamental characterizations of Sidon set, extending the classical result of [HR70, (37.2)] for compact groups. On the other hand, this result establishes the equivalence between the so-called strong Sidon sets (i.e. sets with condition (3) in the theorem below) and Sidon sets in non-amenable discrete groups, which had been open since the work of Picardello [Pic73] in 1970s. Our approach is different from the idea of [HR70, Pic73]. In hindsight, the proof in [HR70] depends essentially on the coamenability of the compact group, which does not apply to the more general cases in the quantum setting. Instead, we use a simpler argument via duality.

Theorem 3.3. Let $\mathbb{G}$ be a compact quantum group and $\mathbf{E}$ be a subset of $\operatorname{Irr}(\mathbb{G})$. The following assertions are equivalent:

(1) $\mathbf{E}$ is a Sidon set;

(2) for any $a \in \ell^{\infty}(\mathbf{E})$, there exists $\varphi \in C_{r}(\mathbb{G})^{*}$ such that $\hat{\varphi}(\pi)=a_{\pi}$ for all $\pi \in \mathbf{E}$;

(3) for any $a \in c_{0}(\mathbf{E})$, there exists $x \in L^{1}(\mathbb{G})$ such that $\hat{x}(\pi)=a_{\pi}$ for all $\pi \in \mathbf{E}$;

(4) $L_{\mathbf{E}}^{\infty}(\mathbb{G}) \subset A(\mathbb{G})$;

(5) $C_{\mathbf{E}}(\mathbb{G}) \subset A(\mathbb{G})$;

(6) there exists $K>0$ such that for any $x \in C_{\mathbf{E}}(\mathbb{G}),\|\hat{x}\|_{1} \leq K\|x\|_{\infty}$;

(7) there exists $K>0$ such that for any $x \in L_{\mathbf{E}}^{\infty}(\mathbb{G}),\|\hat{x}\|_{1} \leq K\|x\|_{\infty}$.

Proof. From Proposition 2.9, it is easy to see that the condition (4) implies the surjectivity of the inverse Fourier transform $\mathcal{F}^{-1}: \ell^{1}(\mathbf{E}) \rightarrow L_{\mathbf{E}}^{\infty}(\mathbb{G})$. Then the equivalence $(4) \Leftrightarrow(7)$ follows from the open mapping theorem. Similarly, we may obtain $(5) \Leftrightarrow(6)$. Also, the implications $(7) \Rightarrow(6) \Rightarrow(1)$ are trivial. Let us show $(1) \Rightarrow(2) \Rightarrow(3) \Rightarrow(1)$ and $(3) \Rightarrow(7)$. In the following set $\operatorname{Pol}_{\mathbf{E}}(\mathbb{G})^{c}=\left\{x \in \operatorname{Pol}(\mathbb{G}): x^{*} \in \operatorname{Pol}_{\mathbf{E}}(\mathbb{G})\right\}$.

$(1) \Rightarrow(2)$. Take $a \in \ell^{\infty}(\mathbf{E})$. We consider the functional

$$
\varphi: \operatorname{Pol}_{\mathbf{E}}(\mathbb{G})^{c} \rightarrow \mathbb{C}, \quad x \mapsto \overline{\hat{h}\left(a^{*} x^{*}\right)}
$$

According to (1), we have

$$
|\varphi(x)|=\left|\hat{h}\left(a^{*} \widehat{x^{*}}\right)\right| \leq\left\|a^{*}\right\|_{\infty}\left\|\widehat{x^{*}}\right\|_{1} \leq K\left\|a^{*}\right\|_{\infty}\left\|x^{*}\right\|_{\infty}=K\|a\|_{\infty}\|x\|_{\infty}
$$

so $\varphi$ is continuous on $\operatorname{Pol}_{\mathbf{E}}(\mathbb{G})^{c}$ and it has a Hahn-Banach extension to $C_{r}(\mathbb{G})$. We still denote the extension by $\varphi$. Recall that by Proposition 2.2 ,

$$
\left(u_{j i}^{(\pi)}\right)^{\wedge}(\pi)=d_{\pi}^{-1} e_{i j}^{(\pi)} Q_{\pi}^{-1}
$$


We have for $\pi \in \mathbf{E}$,

$$
\begin{aligned}
\hat{\varphi}(\pi) & =(\varphi \otimes \iota)\left(\left(u^{(\pi)}\right)^{*}\right)=\sum_{1 \leq i, j \leq n(\pi)} \varphi\left(\left(u_{j i}^{(\pi)}\right)^{*}\right) e_{i j}^{(\pi)} \\
& =\sum_{1 \leq i, j \leq n(\pi)} \overline{\hat{h}\left(a^{*}\left(u_{j i}^{(\pi)}\right)^{\wedge}(\pi)\right)} e_{i j}^{(\pi)}=d_{\pi}^{-1} \sum_{1 \leq i, j \leq n(\pi)} \overline{\hat{h}\left(a^{*} e_{i j}^{(\pi)} Q_{\pi}^{-1}\right)} e_{i j}^{(\pi)} \\
& =\sum_{1 \leq i, j \leq n(\pi)} \overline{\operatorname{Tr}\left(a_{\pi}^{*} e_{i j}^{(\pi)}\right)} e_{i j}^{(\pi)}=\sum_{1 \leq i, j \leq n(\pi)} \overline{\left(a_{\pi}^{*}\right)_{j i}} e_{i j}^{(\pi)}=a_{\pi},
\end{aligned}
$$

as desired.

$(2) \Rightarrow(3)$. We consider the map

$$
\sigma: L^{1}(\mathbb{G}) \rightarrow c_{0}(\mathbf{E}),\left.\quad \psi \mapsto \hat{\psi}\right|_{\mathbf{E}}
$$

Then the second adjoint map $\left(\sigma^{*}\right)^{*}$ is given by

$$
\sigma^{* *}: L^{\infty}(\mathbb{G})^{*} \rightarrow \ell^{\infty}(\mathbf{E}),\left.\quad \psi \mapsto \hat{\psi}\right|_{\mathbf{E}}
$$

Note that the condition (2) means nothing but the surjectivity of $\sigma^{* *}$. Recall the general fact that for a bounded map $T$ between two Banach spaces, $T$ is surjective if and only if $T^{* *}$ is surjective (cf. for example [Meg98, 3.1.22]). So $\sigma$ is also surjective, whence the condition (3).

$(3) \Rightarrow(1)$. The assertion (3) implies that the bounded map $\sigma:\left.\psi \mapsto \hat{\psi}\right|_{\mathbf{E}}$ from $L^{1}(\mathbb{G})$ to $c_{0}(\mathbf{E})$ is surjective. By the open mapping theorem, we may find a constant $K>0$ such that for all $a \in c_{0}(\mathbf{E})$, there exists $x \in L^{1}(\mathbb{G})$ satisfying $\hat{x}(\pi)=a_{\pi}$ for all $\pi \in \mathbf{E}$ and $\|x\|_{1} \leq K\|a\|_{\infty}$.

Now consider $y \in \operatorname{Pol}_{\mathbf{E}}(\mathbb{G})$ and let us show that $\|\hat{y}\|_{1} \leq K\|y\|_{\infty}$. Equivalently, let us prove that for all $a \in c_{c}(\mathbf{E})$ with $\|a\|_{\infty} \leq 1$, we have

$$
\left|\hat{h}\left(a^{*} \hat{y}\right)\right| \leq K\|y\|_{\infty} .
$$

In the following let $y$ and $a$ be fixed as above. Choose a $\psi \in L^{1}(\mathbb{G})$ satisfying $\hat{\psi}(\pi)=a_{\pi}$ for all $\pi \in \mathbf{E}$ and $\|\psi\|_{1} \leq K$. Note also that $\left.\psi\right|_{\operatorname{Pol}_{\mathbf{E}}(\mathbb{G})^{c}}=\left.\left(\mathcal{F}^{-1}(a) h\right)\right|_{\operatorname{Pol}_{\mathbf{E}}(\mathbb{G})^{c}}$. Hence together with Proposition 2.2 and the choice of $y$, we get

$$
\left|\hat{h}\left(a^{*} \hat{y}\right)\right|=\left|\hat{h}\left(\hat{y}^{*} a\right)\right|=\left|h\left(y^{*} \mathcal{F}^{-1}(a)\right)+\left(\psi-\mathcal{F}^{-1}(a) h\right)\left(y^{*}\right)\right|=\left|\psi\left(y^{*}\right)\right| \leq K\|y\|_{\infty} .
$$

Therefore the desired inequality (3.1) follows.

$(3) \Rightarrow(7)$. We have proved that (3) implies (1) and on the other hand, for any set $\mathbf{E} \subset \operatorname{Irr}(\mathbb{G})$ satisfying (1), we have the following observation: if $\psi \in L^{1}(\mathbb{G})$ satisfies $\left.\hat{\psi}\right|_{\mathbf{E}}=0$, then for all $x \in L_{\mathbf{E}}^{\infty}(\mathbb{G})$, we have $\psi\left(x^{*}\right)=0$. In fact, let $\left(y_{n}\right) \subset \operatorname{Pol}(\mathbb{G})$ be a sequence such that $y_{n} h$ converges to $\psi$ in $L^{1}(\mathbb{G})$ and let $x \in L_{\mathbf{E}}^{\infty}(\mathbb{G})$. We then note that $\hat{\psi}^{*} \hat{x}=0,\left(\hat{y}_{n}-\hat{\psi}\right)^{*} \hat{x} \in c_{c}(\mathbf{E})$ and by $(2.2)$ and (2.4) we have

$$
\begin{aligned}
\left|\hat{h}\left(\hat{y}_{n}^{*} \hat{x}\right)\right| & =\left|\hat{h}\left(\left(\hat{y}_{n}-\hat{\psi}\right)^{*} \hat{x}\right)\right| \leq\left\|\left(\hat{y}_{n}-\hat{\psi}\right)^{*} \hat{x}\right\|_{1}=\left\|\mathcal{F}\left(\left(\iota \otimes\left(y_{n} h-\psi\right)^{*}\right) \Delta(x)\right)\right\|_{1} \\
& \leq K\left\|\left(\iota \otimes\left(y_{n} h-\psi\right)^{*}\right) \Delta(x)\right\|_{\infty} \leq K\left\|y_{n} h-\psi\right\|_{1}\|x\|_{\infty} \rightarrow 0
\end{aligned}
$$

where $K$ is the Sidon constant for E. Consequently by the choice of $y_{n}$ and Proposition 2.2 we have

$$
\psi\left(x^{*}\right)=\lim _{n} h\left(x^{*} y_{n}\right)=\lim _{n} \overline{\hat{h}\left(\hat{y}_{n}^{*} \hat{x}\right)}=0,
$$

as claimed. Then the implication $(3) \Rightarrow(7)$ follows from the same argument as in $(3) \Rightarrow(1)$ above.

Remark 3.4. Note that for a Sidon set E, by the assertion (4) and Proposition 2.9, we have

$$
L_{\mathbf{E}}^{\infty}(\mathbb{G})=C_{\mathbf{E}}(\mathbb{G}) \text {. }
$$

By the assertion $(7), C_{\mathbf{E}}(\mathbb{G})$ is isomorphic to a Banach subspace of $\ell^{1}(\mathbf{E})$ via the Fourier transform. Since $c_{c}(\mathbf{E})$ is dense in $\ell^{1}(\mathbf{E}), C_{\mathbf{E}}(\mathbb{G})$ and $\ell^{1}(\mathbf{E})$ are indeed isomorphic. In particular, the subspace $\operatorname{Pol}_{\mathbf{E}}(\mathbb{G})$ is dense in $C_{\mathbf{E}}(\mathbb{G})$. 
Remark 3.5. Since by $(2.2)$ we know that $\left(\varphi^{*} \circ S^{-1}\right)^{\wedge}(\pi)=\hat{\varphi}(\pi)^{*}$ for $\pi \in \operatorname{Irr}(\mathbb{G})$, the assertion $(2)$ in the above theorem can be replaced by the following one:

$\left(2^{\prime}\right)$ for any $a \in \ell^{\infty}(\mathbf{E})$, there exists $\varphi \in C_{r}(\mathbb{G})^{*}$ such that $\left(\varphi \circ S^{-1}\right)^{\wedge}(\pi)=a_{\pi}$ for all $\pi \in \mathbf{E}$.

Remark 3.6. Note that we have shown in the proof that, if $\mathbf{E}$ is a Sidon set of constant $K$, then the obtained elements $\varphi$ and $x$ in (2) and (3) can be chosen to have the norms not more than $K\|a\|_{\infty}$, respectively; conversely arguing as in the beginning of the proof $(3) \Rightarrow(1)$, if $(2)$ or $(3)$ holds, we may find a constant $K>0$ such that the norms of $\varphi$ or $x$ is not more than $K\|a\|_{\infty}$ respectively, and the obtained Sidon constant in (1) is exactly $K$.

Remark 3.7. In view of some technical tricks concerning the non-traciality of Haar states, we would like to present a second proof of the implication $(1) \Rightarrow(7)$ in the above theorem under an additional assumption that $\mathbb{G}$ is coamenable, roughly following the idea in $[\mathrm{HR} 70,(37.2)]$. The subtle point, which is trivially hidden in the commutative and cocommutative cases, is the fact that to directly deduce the convergence of Fourier series of $x$ from (3.2) below as in [HR70, (37.2)], one needs to know that the convolution $(1.7)$ defines a bounded map from $L^{1}(\mathbb{G}) \times L^{\infty}(\mathbb{G})$ into $L^{\infty}(\mathbb{G})$ by restriction. This is generally not clear for a compact quantum group which is not of Kac type, caused by the unboundedness of the antipode $S$. Now let us assume the coamenability of $\mathbb{G}$, and show the implication $(1) \Rightarrow(7)$ :

Assume (1). Take $x \in L_{\mathbf{E}}^{\infty}(\mathbb{G})$. Since $\mathbb{G}$ is coamenable and the subspace $\operatorname{Pol}(\mathbb{G})$ is dense in $L^{1}(\mathbb{G})$, we may choose a net $\left(y_{i}\right)_{i \in I} \subset \operatorname{Pol}(\mathbb{G})$ with $\left\|y_{i}\right\|_{1} \leq 1$ such that $\lim _{i}\left\|x \star y_{i}-x\right\|_{1}=0$. Hence for each $\pi \in \mathbf{E}$,

$$
\left\|\hat{y}_{i}(\pi) \hat{x}(\pi)-\hat{x}(\pi)\right\|_{B\left(H_{\pi}\right)} \leq\left\|\hat{y}_{i} \hat{x}-\hat{x}\right\|_{\infty} \leq\left\|x \star y_{i}-x\right\|_{1} \rightarrow 0 .
$$

Since the norms on a finite dimensional space are equivalent, we have for all $\pi \in \mathbf{E}$,

$$
\lim _{i} \operatorname{Tr}\left(\left|\left(\hat{y}_{i}(\pi) \hat{x}(\pi)-\hat{x}(\pi)\right) Q_{\pi}\right|\right)=0 \text {. }
$$

Therefore for any finite subset $\mathbf{F} \subset \mathbf{E}$ and for any $\varepsilon>0$, we may find some $a \in c_{c}(\mathbf{E})$ with $\|a\|_{\infty} \leq 1$ and

$$
\sum_{\pi \in \mathbf{F}} d_{\pi} \operatorname{Tr}\left(\left|\left(a_{\pi} \hat{x}(\pi)-\hat{x}(\pi)\right) Q_{\pi}\right|\right)<\varepsilon
$$

Since $\mathbf{E}$ is a Sidon set, we may find by previous arguments (proof of $(1) \Rightarrow(2) \Rightarrow(3)$ ) a functional $\varphi \in L^{\infty}(\mathbb{G})_{*}=L^{1}(\mathbb{G})$ with $\|\varphi\|_{1} \leq K$ and $\hat{\varphi}(\pi)=a_{\pi}^{*}$ for all $\pi \in \mathbf{E}$. Take $y \in \operatorname{Pol}(\mathbb{G})$ such that $\|\varphi-y\|_{1}<\varepsilon$. Then $\left\|a_{\pi}-\left((y h)^{*} \circ S^{-1}\right)^{\wedge}(\pi)\right\|_{B\left(H_{\pi}\right)}=\left\|\hat{\varphi}(\pi)^{*}-\hat{y}(\pi)^{*}\right\|_{B\left(H_{\pi}\right)}<\varepsilon$ for $\pi \in \mathbf{E}$. Further together with (2.4),

$$
\begin{aligned}
\sum_{\pi \in \mathbf{F}} d_{\pi} \operatorname{Tr}\left(\left|\hat{x}(\pi) Q_{\pi}\right|\right) & \leq \sum_{\pi \in \mathbf{F}} d_{\pi} \operatorname{Tr}\left(\left|a_{\pi} \hat{x}(\pi) Q_{\pi}\right|\right)+\sum_{\pi \in \mathbf{F}} d_{\pi} \operatorname{Tr}\left(\left|\left(a_{\pi} \hat{x}(\pi)-\hat{x}(\pi)\right) Q_{\pi}\right|\right) \\
& \leq \sum_{\pi \in \mathbf{F}} d_{\pi} \operatorname{Tr}\left(\left|\left((y h)^{*} \circ S^{-1}\right)^{\wedge}(\pi) \hat{x}(\pi) Q_{\pi}\right|\right)+\varepsilon \sum_{\pi \in \mathbf{F}} d_{\pi} \operatorname{Tr}\left(\mid\left(\hat{x}(\pi) Q_{\pi} \mid\right)+\varepsilon\right. \\
& \leq\left\|\mathcal{F}\left(\left(\iota \otimes(y h)^{*}\right) \Delta(x)\right)\right\|_{1}+\varepsilon \sum_{\pi \in \mathbf{F}} d_{\pi} \operatorname{Tr}\left(\mid\left(\hat{x}(\pi) Q_{\pi} \mid\right)+\varepsilon\right. \\
& \leq K\left\|\left(\iota \otimes(y h)^{*}\right) \Delta(x)\right\|_{\infty}+\varepsilon \sum_{\pi \in \mathbf{F}} d_{\pi} \operatorname{Tr}\left(\mid\left(\hat{x}(\pi) Q_{\pi} \mid\right)+\varepsilon\right. \\
& \leq K(K+\varepsilon)\|x\|_{\infty}+\varepsilon \sum_{\pi \in \mathbf{F}} d_{\pi} \operatorname{Tr}\left(\mid\left(\hat{x}(\pi) Q_{\pi} \mid\right)+\varepsilon\right.
\end{aligned}
$$

where we have applied the property of the Sidon set to the element $\left(\iota \otimes(y h)^{*}\right) \Delta(x) \in \operatorname{Pol}_{\mathbf{E}}(\mathbb{G})$. Since $\mathbf{F}$ and $\varepsilon$ are arbitrarily chosen, we get $\|\hat{x}\|_{1} \leq K^{2}\|x\|_{\infty}$, as desired. Note that the constant $K^{2}$ obtained here is worse than that in the previous proof.

As a corollary we may give a quick proof of the non-surjectivity of the Fourier transform $\mathcal{F}: L^{1}(\mathbb{G}) \rightarrow c_{0}(\hat{\mathbb{G}})$ for infinite compact quantum group $\mathbb{G}$. 
Corollary 3.8. Let $\mathbb{G}$ be a compact quantum group. The following conditions are equivalent:

$(1) \mathbb{G}$ is finite, i.e., $L^{\infty}(\mathbb{G})$ is a finite-dimensional space;

$(2) \operatorname{Irr}(\mathbb{G})$ is a Sidon set;

(3) $\mathcal{F}: L^{1}(\mathbb{G}) \rightarrow c_{0}(\hat{\mathbb{G}})$ is surjective;

(4) $\mathcal{F}: L^{\infty}(\mathbb{G})^{*} \rightarrow \ell^{\infty}(\hat{\mathbb{G}})$ is surjective.

Proof. The equivalence $(2) \Leftrightarrow(3) \Leftrightarrow(4)$ has been already given in Theorem 3.3. The implication $(1) \Rightarrow(2)$ is trivial. Assume (2) holds, then the previous theorem yields that there exists a constant $K>0$ such that

$$
\forall x \in L^{\infty}(\mathbb{G}), \quad\|x\|_{\infty} \leq\|\hat{x}\|_{1} \leq K\|x\|_{\infty} .
$$

Suppose by contradiction with $(1)$ that $\mathbb{G}$ is not finite. Then we may choose an infinite countable subset $\mathbf{E} \subset \operatorname{Irr}(\mathbb{G})$ and let $A$ be the $*$-subalgebra generated by $\left\{\chi_{\pi}: \pi \in \mathbf{E}\right\}$ in $\operatorname{Pol}(\mathbb{G})$. Write

$$
\mathbf{F}=\left\{\pi \in \operatorname{Irr}(\mathbb{G}): \exists \pi_{1}, \ldots, \pi_{n} \in \mathbf{E}, \pi \text { is a subrepresentation of } \sigma_{1} \otimes \cdots \otimes \sigma_{n}, \sigma_{i}=\pi_{i} \text { or } \bar{\pi}_{i}\right\},
$$

then $\mathbf{F}$ is countable and $A \subset \operatorname{Pol}_{\mathbf{F}}(\mathbb{G})$. Consider the von Neumann subalgebra $\mathcal{M}$ generated by $A$ in $L^{\infty}(\mathbb{G})$. Then by the weak density of $A$ in $\mathcal{M}$, for each $x \in \mathcal{M}$ and each $\pi \in \operatorname{Irr}(\mathbb{G}) \backslash \mathbf{F}$ we have $\hat{x}(\pi)=0$. So by the above inequality (3.3) each $x \in \mathcal{M}$ can be approximated in \|\|$_{\infty}$ by elements in $\operatorname{Pol}_{\mathbf{F}}(\mathbb{G})$, and in particular $\mathcal{M}$ is separable, which gives a contradiction since the von Neumann algebra $\mathcal{M}$ is infinite-dimensional as so is $A$.

Remark 3.9. Together with the condition (5) in Theorem 3.3, the above argument also shows that for any infinite discrete quantum group $\mathbb{H}$, the Fourier transform $\mathcal{F}: \ell^{1}(\mathbb{H}) \rightarrow C(\hat{\mathbb{H}})$ in the sense of [Kah10, Cas13] is not surjective. The above result is a particular case of the general fact that the predual of an infinite-dimensional von Neumann algebra cannot be equipped with an equivalent $\mathrm{C}^{*}$-norm.

The following properties give some general methods of constructing infinite Sidon sets for compact quantum groups.

Proposition 3.10. Let $\left(\mathbb{G}_{i}\right)_{i \in I}$ be a family of compact quantum groups and assume that $\mathbf{E}_{i} \subset$ $\operatorname{Irr}\left(\mathbb{G}_{i}\right)$ is a Sidon set with constant $C_{i}$ for each $i \in I$ and that $C:=\sup _{i \in I} C_{i}<\infty$. Then $\cup_{i \in I} \mathbf{E}_{i} \subset \operatorname{Irr}\left(\prod_{i \in I} \mathbb{G}_{i}\right)$ is a Sidon set for $\prod_{i \in I} \mathbb{G}_{i}$.

Proof. This follows directly from the assertion (2) in Theorem 3.3 and Remark 3.6. Let $a \in$ $\ell^{\infty}\left(\cup_{i \in I} \mathbf{E}_{i}\right)$. Without loss of generality we assume $\|a\|_{\infty}=1 / C$. For each $i \in I$, we may find $\varphi_{i} \in C_{r}\left(\mathbb{G}_{i}\right)^{*}$ such that $\hat{\varphi}_{i}$ coincides with $a$ on $\mathbf{E}_{i}$ and $\left\|\varphi_{i}\right\| \leq 1$. Take $\varphi=\otimes_{i \in I} \varphi_{i}$, then $\varphi$ extends to a bounded functional on $C_{r}\left(\prod_{i \in I} \mathbb{G}_{i}\right)$. Hence $\hat{\varphi}(\pi)=a_{\pi}$ for all $\pi \in \cup_{i \in I} \mathbf{E}_{i}$. So $\cup_{i \in I} \mathbf{E}_{i}$ is a Sidon set.

Proposition 3.11. Let $\mathbb{G}_{1}, \mathbb{G}_{2}$ be compact quantum groups. Assume that $\mathbf{E} \subset \operatorname{Irr}\left(\mathbb{G}_{1}\right)$ and $\mathbf{F} \subset$ $\operatorname{Irr}\left(\mathbb{G}_{2}\right)$ are Sidon sets. Then $\mathbf{E} \cup \mathbf{F}$ is a Sidon set for $\mathbb{G}_{1} \hat{*} \mathbb{G}_{2}$.

Proof. Denote $h_{1}$ and $h_{2}$ the Haar states for $\mathbb{G}_{1}$ and $\mathbb{G}_{2}$ respectively. Let $K_{1}$ be the Sidon constant for $\mathbf{E}$ and $K_{2}$ that for $\mathbf{F}$. By Remark 3.2, we may assume that $1 \notin \mathbf{E}$ and $1 \notin \mathbf{F}$. Now for any $x \in \operatorname{Pol}_{\mathbf{E}}\left(\mathbb{G}_{1} \hat{*} \mathbb{G}_{2}\right)$ and $y \in \operatorname{Pol}_{\mathbf{F}}\left(\mathbb{G}_{1} \hat{*} \mathbb{G}_{2}\right)$, we have $h_{1}(x)=0, h_{2}(y)=0$, and $x$ and $y$ are free. Then it is well known and easy to see from the construction of reduced free products that $\max \left\{\|x\|_{\infty},\|y\|_{\infty}\right\} \leq\|x+y\|_{\infty}$ (see [Voi98, Jun05, RX06] for more information on the norm estimates related to freeness). Hence

$$
\|\hat{x}+\hat{y}\|_{1} \leq\|\hat{x}\|_{1}+\|\hat{y}\|_{1} \leq K_{1}\|x\|_{\infty}+K_{2}\|y\|_{\infty} \leq\left(K_{1}+K_{2}\right)\|x+y\|_{\infty} .
$$

This proves that $\mathbf{E} \cup \mathbf{F}$ is a Sidon set of constant $K_{1}+K_{2}$.

Remark 3.12. Note that one cannot expect to extend the above proposition to an infinite family of compact quantum groups as in Proposition 3.10. An easy example is the set of infinitely many free generators of the free group $\mathbb{F}_{\infty}$, which is not a Sidon set. More details of this example will be presented in Remark 3.16. 
As is seen in [Pic73, Boż81], there are several alternative possible ways to generalize the notion of Sidon sets for non-amenable cases. Let us briefly discuss them in the quantum group setting. We follow the terminologies in [Boż81, Har99].

Definition 3.13. (1) We say that a subset $\mathbf{E} \subset \operatorname{Irr}(\mathbb{G})$ is a weak Sidon set (with constant $K$ ) if there exists $K>0$ such that for any $x \in \operatorname{Pol}_{\mathbf{E}}(\mathbb{G})$, we have

$$
\|\hat{x}\|_{1} \leq K\|x\|_{C_{u}(\mathbb{G})} .
$$

(2) We say that $\mathbf{E} \subset \operatorname{Irr}(\mathbb{G})$ is an interpolation set of $\mathrm{M}\left(L^{\infty}(\mathbb{G})\right.$ ) (resp., of $\mathrm{M}_{L}\left(L^{\infty}(\mathbb{G})\right)$, of $\mathrm{M}_{R}\left(L^{\infty}(\mathbb{G})\right)$ ) with constant $K$ if for any $a \in \ell^{\infty}(\mathbf{E})$, there exists a bounded multiplier $\tilde{a} \in$ $\mathrm{M}\left(L^{\infty}(\mathbb{G})\right)\left(\right.$ resp., $\left.\tilde{a} \in \mathrm{M}_{L}\left(L^{\infty}(\mathbb{G})\right), \tilde{a} \in \mathrm{M}_{R}\left(L^{\infty}(\mathbb{G})\right)\right)$ with $\|\tilde{a}\|_{\mathrm{M}\left(L^{\infty}(\mathbb{G})\right)} \leq K\|a\|_{\infty}\left(\operatorname{resp} .,\left\|m_{\tilde{a}}^{L}\right\| \leq\right.$ $\left.K\|a\|_{\infty},\left\|m_{\tilde{a}}^{R}\right\| \leq K\|a\|_{\infty}\right)$ such that $\tilde{a}_{\pi}=a_{\pi}$ for all $\pi \in \mathbf{E}$.

(3) We say that a subset $\mathbf{E} \subset \operatorname{Irr}(\mathbb{G})$ is a left (resp., right) unconditional Sidon set (with constant $K)$ if there exists $K>0$ such that for any unitary $a \in \ell^{\infty}(\mathbf{E})$ and for any $x \in \operatorname{Pol}_{\mathbf{E}}(\mathbb{G})$, $\left\|m_{a}^{L} x\right\|_{\infty} \leq K\|x\|_{\infty}$ (resp., $\left.\left\|m_{a}^{R} x\right\|_{\infty} \leq K\|x\|_{\infty}\right)$.

Remark 3.14. (1) Following the same idea as in the proof $(1) \Rightarrow(2)$ and $(3) \Rightarrow(1)$ in Theorem 3.3, one can see easily that a subset $\mathbf{E} \subset \operatorname{Irr}(\mathbb{G})$ is a weak Sidon set of constant $K$ if and only if for all $a \in \ell^{\infty}(\mathbf{E})$, there exists $\varphi \in C_{u}(\mathbb{G})^{*}$ such that $\|\varphi\| \leq K\|a\|_{\infty}$ and $\hat{\varphi}(\pi)=a_{\pi}$ for all $\pi \in \mathbf{E}$. Evidently, a Sidon set for $\mathbb{G}$ is necessarily a weak Sidon set.

(2) If $\mathbb{G}$ is of Kac type, by Lemma 2.5 we see that the classes of interpolation sets of $\mathrm{M}_{L}\left(L^{\infty}(\mathbb{G})\right)$ and of $\mathrm{M}_{R}\left(L^{\infty}(\mathbb{G})\right)$ coincide. Also we note that if $\mathbb{G}$ is coamenable, these two classes coincide as well, which can be seen from the following theorem. Some more properties of these classes of interpolation sets will be discussed in the next section.

Theorem 3.15. Let $\mathbb{G}$ be a compact quantum group and $\mathbf{E} \subset \operatorname{Irr}(\mathbb{G})$ be a subset. Let $K>0$. If

(1) $\mathbf{E}$ is a weak Sidon set of constant $K$; then

(2) $\mathbf{E}$ is an interpolation set of $\mathrm{M}\left(L^{\infty}(\mathbb{G})\right)$ of constant $K$; and in particular,

(3) $\mathbf{E}$ is a left unconditional Sidon set of constant $K$.

Moreover, if additionally $\mathbb{G}$ is coamenable, then the conditions (1), (2) and (3) are all equivalent to:

(4) $\mathbf{E}$ is a Sidon set of constant $K$.

Proof. The proof follows the same line as in [Pic73, Boż81] and we only present the sketch. Assume that $\mathbf{E} \subset \operatorname{Irr}(\mathbb{G})$ is a weak Sidon set. Then by the above remark and Remarks 3.5-3.6 any $a \in \ell^{\infty}(\mathbf{E})$ is a restriction of $\hat{\varphi}$ for some $\varphi \in C_{u}(\mathbb{G})^{*}$ of norm no more than $K\|a\|_{\infty}$. Note that $C_{r}(\mathbb{G})$ is a quotient space of $C_{u}(\mathbb{G})$ and hence the dual space $C_{r}(\mathbb{G})^{*}$ embeds isometrically into $C_{u}(\mathbb{G})^{*}$. Therefore by the density of $\operatorname{Pol}(\mathbb{G})$ in $L^{1}(\mathbb{G})=L^{\infty}(\mathbb{G})_{*}$, one can easily see from $(2.3)$ that $\psi \mapsto \varphi \star \psi$ and $\psi \mapsto \psi \star \varphi$ give two bounded multipliers on $L^{1}(\mathbb{G})$. Then by duality we obtain the desired multipliers for (2). Thus the implication $(1) \Rightarrow(2) \Rightarrow(3)$ is established.

Now assume additionally $\mathbb{G}$ is coamenable and show that $(3) \Rightarrow(4)$. Take $x \in \operatorname{Pol}_{\mathbf{E}}(\mathbb{G})$ and for each $\pi \in \mathbf{E}$ let $a_{\pi}$ be a unitary matrix such that $\left|\hat{x}(\pi) Q_{\pi}\right|=a_{\pi} \hat{x}(\pi) Q_{\pi}$. Then

$$
\begin{aligned}
\|\hat{x}\|_{1} & =\sum_{\pi \in \mathbf{E}} d_{\pi} \operatorname{Tr}\left(\left|\hat{x}(\pi) Q_{\pi}\right|\right)=\sum_{\pi \in \mathbf{E}} d_{\pi} \operatorname{Tr}\left(a_{\pi} \hat{x}(\pi) Q_{\pi}\right) \\
& =\epsilon\left(\sum_{\pi \in \mathbf{E}} d_{\pi}(\iota \otimes \operatorname{Tr})\left[\left(1 \otimes a_{\pi} \hat{x}(\pi) Q_{\pi}\right) u^{(\pi)}\right]\right)=\epsilon\left(m_{a} x\right) \\
& \leq\left\|m_{a} x\right\|_{\infty} \leq K\|x\|_{\infty},
\end{aligned}
$$

as desired.

Remark 3.16. (1) The left unconditional Sidon set in the assertion (3) above can be obviously replaced by the right one. 
(2) The coamenability is crucial in the above proposition. In fact, denote by $\mathbb{F}_{\infty}$ the free group with infinitely many generators and let $\mathbb{G}$ be the quantum group with dual $\hat{\mathbb{G}}=\mathbb{F}_{\infty}$. Take $\mathbf{E}$ to be the generators of $\mathbb{F}_{\infty}$, and recall the Haagerup inequality [Lei74, Boż75a]: for finitely many elements $\gamma_{1}, \ldots, \gamma_{n} \in \mathbf{E}$ and $\alpha_{1}, \ldots, \alpha_{n} \in \mathbb{C}$,

$$
\left(\sum_{k=1}^{n}\left|\alpha_{k}\right|^{2}\right)^{1 / 2} \leq\left\|\sum_{k=1}^{n} \alpha_{k} \lambda\left(\gamma_{k}\right)\right\|_{V N\left(\mathbb{F}_{\infty}\right)} \leq 2\left(\sum_{k=1}^{n}\left|\alpha_{k}\right|^{2}\right)^{1 / 2} .
$$

So E satisfies (2) and (3) in the proposition, but in this case obviously (1) fails to hold.

Example 3.17. Consider the compact quantum group $\mathbb{G}=\prod_{k>1} \mathbb{G}_{k}$, where for each $k \geq 1$ and $N_{k} \geq 1, \mathbb{G}_{k}=U_{N_{k}}^{+}$denotes the free unitary group of Wang [VDW96]. Recall that $C_{u}\left(U_{N_{k}}^{+}\right)$is the universal $\mathrm{C}^{*}$-algebra generated by $N_{k}^{2}$ elements $\left\{u_{i j}^{(k)}: 1 \leq i, j \leq N_{k}\right\}$ such that the matrix $u^{(k)}=\left[u_{i j}^{(k)}\right]$ is unitary. Take $\mathbf{E}=\left\{u^{(k)}: k \geq 1\right\} \subset \operatorname{Irr}(\mathbb{G})$. Then $\mathbf{E}$ is a weak Sidon set, and hence is an interpolation set of $\mathrm{M}\left(L^{\infty}(\mathbb{G})\right)$ and an unconditional Sidon set. In fact, let $U_{N_{k}}$ be the $N_{k} \times N_{k}$ unitary matrix group and $w^{(k)}: U_{N_{k}} \rightarrow \mathbb{M}_{N_{k}}(\mathbb{C}), w \mapsto w$ be its fundamental representation. It is easy to see that $\left\{w^{(k)}: k \geq 1\right\}$ is a Sidon set of constant 1 for $G=\prod_{k \geq 1} U_{N_{k}}([\operatorname{HR} 70,(37.5)])$. Thus by the universal property of $U_{N_{k}}^{+}$we have for all finitely supported sequences $\left(A_{k}\right) \in \prod_{k} \mathbb{M}_{N_{k}}$,

$$
\sum_{k \geq 1} \operatorname{Tr}\left(\left|A_{k}\right|\right) \leq\left\|\sum_{k \geq 1} \operatorname{Tr}\left(A_{k} w^{(k)}\right)\right\|_{C(G)} \leq\left\|\sum_{k \geq 1}(\iota \otimes \operatorname{Tr})\left[\left(1 \otimes A_{k}\right) u^{(k)}\right]\right\|_{C_{u}(\mathbb{G})} .
$$

Therefore $\mathbf{E}$ is a weak Sidon set. However, $\mathbf{E}$ is not a Sidon set. Indeed, write $x=\sum_{k} x_{k} \in$ $\operatorname{Pol}_{\mathbf{E}}(\mathbb{G})$ with $x_{k} \in \operatorname{Pol}\left(U_{N_{k}}^{+}\right)$. Vergnioux [Ver07] and Brannan [Bra12, Theorem 6.3] showed that there exists $C>0$ such that $\left\|x_{k}\right\|_{\infty} \leq C\left\|\hat{x}_{k}\right\|_{2}$ for all $k$. Hence $\|x\|_{\infty} \leq C \sum_{k}\left\|\hat{x}_{k}\right\|_{2}$ and the inequality in Definition 3.1 cannot hold.

Example 3.18. Consider the sequence $\left(q_{n}\right)_{n \geq 1} \subset[q, 1]$ with $q:=\inf _{n} q_{n}>0$ and the associated quantum group $\mathbb{G}=\prod_{n \geq 1} \mathrm{SU}_{q_{n}}(2)$. Recall that $\mathrm{SU}_{q_{n}}(2)$ is coamenable and for each $n, C\left(\mathrm{SU}_{q_{n}}(2)\right)$ is the universal $\mathrm{C}^{*}$-algebra generated by elements $\alpha_{n}$ and $\gamma_{n}$ such that the matrix

$$
u_{n}=\left[\begin{array}{cc}
\alpha_{n} & -q_{n} \gamma_{n}^{*} \\
\gamma_{n} & \alpha_{n}^{*}
\end{array}\right]
$$

is unitary. The matrix $u_{n} \in \mathbb{M}_{2}\left(C\left(\mathrm{SU}_{q_{n}}(2)\right)\right)$ defines a unitary representation of $\mathrm{SU}_{q_{n}}(2)$, and the matrix $Q_{u_{n}}$ associated to $u_{n}$, simply written as $Q_{n}$, has the eigenvalues $q_{n}, q_{n}^{-1}$. Let $d_{n}=$ $\operatorname{Tr}\left(Q_{n}\right)=q_{n}+q_{n}^{-1}$. Then $\mathbf{E}=\left\{u_{n}: n \geq 1\right\} \subset \operatorname{Irr}(\mathbb{G})$ is a Sidon set for $\mathbb{G}$. To see this, by Proposition 3.10 and Theorem 3.15, it suffices to show that for each $n \geq 1$, the singleton $\left\{u_{n}\right\}$ has a uniform right unconditional Sidon constant $1+q^{-1}$, which means that for all $A \in \mathbb{M}_{2}(\mathbb{C})$ and all unitaries $V \in \mathbb{M}_{2}(\mathbb{C})$, we have

$$
\left\|d_{n}(\iota \otimes \operatorname{Tr})\left[\left(1 \otimes V A Q_{n}\right) u_{n}\right]\right\|_{\infty} \leq\left(1+q^{-1}\right)\left\|d_{n}(\iota \otimes \operatorname{Tr})\left[\left(1 \otimes A Q_{n}\right) u_{n}\right]\right\|_{\infty} .
$$

Indeed, since the map $x \mapsto d_{n}^{-1}(\iota \otimes \operatorname{Tr})\left(x\left(1 \otimes Q_{n}\right)\right)$ is unital completely positive and the functional $\mathrm{Tr}$ is tracial, we may use the Cauchy-Schwarz inequality and Proposition 2.2 to get

$$
\begin{aligned}
& \left\|d_{n}(\iota \otimes \operatorname{Tr})\left[\left(1 \otimes V A Q_{n}\right) u_{n}\right]\right\|_{\infty}^{2}=\left.d_{n}^{4}\|\| d_{n}^{-1}(\iota \otimes \operatorname{Tr})\left[u_{n}\left(1 \otimes V A Q_{n}\right)\right]\right|^{2} \|_{\infty} \\
\leq & d_{n}^{4}\left\|d_{n}^{-1}(\iota \otimes \operatorname{Tr})\left[\left(u_{n}(1 \otimes V A)\right)^{*}\left(u_{n}(1 \otimes V A)\right)\left(1 \otimes Q_{n}\right)\right]\right\|_{\infty} \\
= & d_{n}^{3} \operatorname{Tr}\left(A^{*} A Q_{n}\right)=d_{n}^{2}\left\|d_{n}(\iota \otimes \operatorname{Tr})\left[\left(1 \otimes A Q_{n}\right) u_{n}\right]\right\|_{2}^{2} \\
\leq & \left(q_{n}+q_{n}^{-1}\right)^{2}\left\|d_{n}(\iota \otimes \operatorname{Tr})\left[\left(1 \otimes A Q_{n}\right) u_{n}\right]\right\|_{\infty}^{2}
\end{aligned}
$$

which establishes (3.4) if we note that $q \leq q_{n} \leq 1$. The order $o\left(q^{-1}\right)$ of the constant obtained in the above inequality is optimal when $q \rightarrow 0$ : we see that

$$
\operatorname{Tr}\left(\left|e_{21}\right|\right)=1, \quad\left\|(\iota \otimes \operatorname{Tr})\left[\left(1 \otimes e_{21}\right) u_{n}\right]\right\|_{\infty}=\left\|-q_{n} \gamma_{n}^{*}\right\| \leq q_{n},
$$

which means the Sidon constant $K$ with $\|\hat{x}\|_{\infty} \leq K\|x\|_{\infty}$ for $x \in \operatorname{Pol}_{\mathbf{E}}(\mathbb{G})$ cannot be less than $q_{n}^{-1}$. Also as a result, if $q_{n} \rightarrow 0$, the subset $\mathbf{E}$ given above is not a Sidon set. 


\section{Relations With $\Lambda(p)$-SETS}

In this section we aim to investigate $\Lambda(p)$-sets, and in particular we will establish the relations between Sidon sets and $\Lambda(p)$-sets.

4.1. $\Lambda(p)$-sets and Sidon sets. In the following we define the $\Lambda(p)$-sets for compact quantum groups, which follows from a direct quantum adaptation of that of classical $\Lambda(p)$-sets for compact groups.

Definition 4.1. Let $\mathbb{G}$ be a compact quantum group and $\mathbf{E} \subset \operatorname{Irr}(\mathbb{G})$ be a subset. Let $\chi_{\pi}=$ $\sum_{i} u_{i i}^{(\pi)}$ be the character of $\pi \in \operatorname{Irr}(\mathbb{G})$. For $1<p<\infty$, we say that $\mathbf{E}$ is a $\Lambda(p)$-set with constant $K$ if there exists $K>0$ such that for all $x \in \operatorname{Pol}_{\mathbf{E}}(\mathbb{G})$,

$$
\|x\|_{p} \leq K\|x\|_{1}
$$

and we say that $\mathbf{E}$ is a central $\Lambda(p)$-set with constant $K$ if there exists $K>0$ such that for all finitely supported sequences $\left(c_{\pi}\right)_{\pi \in \mathbf{E}} \subset \mathbb{C}$ and $x=\sum_{\pi \in \mathbf{E}} c_{\pi} \chi_{\pi} \in \operatorname{Pol}_{\mathbf{E}}(\mathbb{G})$,

$$
\|x\|_{p} \leq K\|x\|_{1} \text {. }
$$

Remark 4.2. Let $1<p<\infty$ and $1<p_{0}<p$. Notice that in order to see a subset $\mathbf{E} \subset \operatorname{Irr}(\mathbb{G})$ is a $\Lambda(p)$-set, it suffices to check the existence of a constant $K>0$ with

$$
\|x\|_{p} \leq K\|x\|_{p_{0}}, \quad x \in \operatorname{Pol}_{\mathbf{E}}(\mathbb{G}) .
$$

This is due to the fact that $\left(L^{p}(\mathbb{G})\right)_{1 \leq p \leq \infty}$ is a complex interpolation scale so that $\|x\|_{p_{0}} \leq$ $\|x\|_{1}^{\theta}\|x\|_{p}^{1-\theta}$ for some $0<\theta<1$. On the other hand, we see that any $\Lambda(p)$-set must be a $\Lambda\left(p^{\prime}\right)$-set for $1<p^{\prime}<p<\infty$. Similar observations are also valid for central $\Lambda(p)$-sets. And as in the classical case, we will be mainly interested in the $\Lambda(p)$-sets for $2<p<\infty$.

It is well known and not difficult to see that when $\mathbb{G}$ is a compact group $G$ or the dual quantum group of a discrete group $\Gamma$, any Sidon set $\mathbf{E} \subset \operatorname{Irr}(\mathbb{G})$ (or more generally, an interpolation set of $\left.\mathrm{M}\left(L^{\infty}(\mathbb{G})\right)\right)$ is a $\Lambda(p)$-set for $1<p<\infty$ ([HR70, Har99]). The same question for an arbitrary compact quantum group is however more delicate. To the best knowledge of the author, the only effort towards this direction before our work is the following property recently given by Blendek and Michaliček, as a main result in [BM13]: if $\mathbb{G}$ is a compact quantum group of Kac type and if $\mathbf{E} \subset \operatorname{Irr}(\mathbb{G})$ is a Sidon set satisfying the Helgason-Sidon condition, then there exists $K>0$ such that for all finitely supported sequences $\left(c_{\pi}\right)_{\pi \in \mathbf{E}} \subset \mathbb{C}$ and $x=\sum_{\pi \in \mathbf{E}} c_{\pi} \chi_{\pi} \in \mathrm{Pol}_{\mathbf{E}}(\mathbb{G})$,

$$
\|x\|_{2} \leq K\|x\|_{1} \text {. }
$$

Observe that this result requires many more restrictions on the subset $\mathbf{E}$ than in the classical cases while the obtained inequality is much weaker. However, compared to the classical one, its proof utilizes quite nontrivial tools such as "modified Rademacher functions". In the following paragraph, we provide an alternative and more concise argument, which completely removes the non-expected restrictions in [BM13] and moreover directly establishes the nice relation between Sidon sets and $\Lambda(p)$-sets for all $1<p<\infty$.

In order to characterize the $\Lambda(p)$-sets, let us consider the following notions of interpolation sets of bounded multipliers on $L^{p}(\mathbb{G})$, which also generalize Definition 3.13.

Definition 4.3. Let $\mathbb{G}$ be a compact quantum group and let $1 \leq p \leq \infty$. We say that $\mathbf{E} \subset \operatorname{Irr}(\mathbb{G})$ is an interpolation set of $\mathrm{M}\left(L^{p}(\mathbb{G})\right)$ (resp., of $\mathrm{M}_{L}\left(L^{p}(\mathbb{G})\right)$, of $\mathrm{M}_{R}\left(L^{\infty}(\mathbb{G})\right)$ ) with constant $K$ if for any $a \in \ell^{\infty}(\mathbf{E})$, there exists a bounded multiplier $\tilde{a} \in \mathrm{M}\left(L^{p}(\mathbb{G})\right.$ ) (resp., $\tilde{a} \in \mathrm{M}_{L}\left(L^{p}(\mathbb{G})\right)$, $\left.\tilde{a} \in \mathrm{M}_{R}\left(L^{p}(\mathbb{G})\right)\right)$ with $\|\tilde{a}\|_{\mathrm{M}\left(L^{p}(\mathbb{G})\right)} \leq K\|a\|_{\infty}\left(\right.$ resp., $\left\|m_{\tilde{a}}^{L}\right\|_{B\left(L^{p}(\mathbb{G})\right)} \leq K\|a\|_{\infty},\left\|m_{\tilde{a}}^{R}\right\|_{B\left(L^{p}(\mathbb{G})\right)} \leq$ $\left.K\|a\|_{\infty}\right)$ such that $\tilde{a}_{\pi}=a_{\pi}$ for all $\pi \in \mathbf{E}$.

Remark 4.4. Let $1 \leq p \leq \infty$ and $\mathbf{E} \subset \operatorname{Irr}(\mathbb{G})$. We remark that, if for any $a \in \ell^{\infty}(\mathbf{E})$, there exists a bounded multiplier $\tilde{a} \in \mathrm{M}\left(L^{p}(\mathbb{G})\right)$, then automatically there exists a constant $K>0$ with $\|\tilde{a}\|_{\mathrm{M}\left(L^{p}(\mathbb{G})\right)} \leq K\|a\|_{\infty}$, and $\mathbf{E}$ is an interpolation set of $\mathrm{M}\left(L^{p}(\mathbb{G})\right)$. In fact, if for any $a \in \ell^{\infty}(\mathbf{E})$, there exists a bounded multiplier $\tilde{a} \in \mathrm{M}\left(L^{p}(\mathbb{G})\right)$ with $\tilde{a}_{\pi}=a_{\pi}$ for all $\pi \in \mathbf{E}$, then by Proposition 2.7 , we have

$$
\left\|Q^{\frac{1}{4}-\frac{1}{2 p}} a Q^{-\frac{1}{4}+\frac{1}{2 p}}\right\|_{\infty}<\infty .
$$


In particular, we choose an appropriate basis of $\oplus_{\pi} H_{\pi}$ so that $Q_{\pi}$ is diagonal under this basis for $\pi \in \operatorname{Irr}(\mathbb{G})$, and take $a_{\pi}=e_{i j}$ with $1 \leq i, j \leq n_{\pi}$, then the above inequality yields that

$$
\sup _{\pi \in \mathbf{E}}\left\|Q_{\pi}\right\|<\infty, \quad \sup _{\pi \in \mathbf{E}}\left\|Q_{\pi}^{-1}\right\|<\infty
$$

Also by Proposition 2.7, it is easy to see that $\mathrm{M}\left(L^{p}(\mathbb{G})\right)$ is a Banach subspace of $B\left(L^{p}(\mathbb{G})\right)$. So by the open mapping theorem and Proposition 2.7, we may always find a constant $K>0$ such that the inequality

$$
\|\tilde{a}\|_{\mathrm{M}\left(L^{p}(\mathbb{G})\right)} \leq K\|a\|_{\infty}
$$

is automatically satisfied. Thus $\mathbf{E}$ is automatically an interpolation set of $\mathrm{M}\left(L^{p}(\mathbb{G})\right)$. But we do not know whether the similar observation can be made for interpolation sets of $\mathrm{M}_{L}\left(L^{p}(\mathbb{G})\right)$ and that of $\mathrm{M}_{R}\left(L^{p}(\mathbb{G})\right)$.

These kinds of lacunarities have some special restrictive properties in the non-Kac case. The following result will be of use later.

Proposition 4.5. Let $\mathbb{G}$ be a compact quantum group and $1 \leq p \leq \infty$. Assume that $\mathbf{E} \subset \operatorname{Irr}(\mathbb{G})$ satisfies one of the following four conditions:

(1) $p \neq \infty$ and $\mathbf{E} \subset \operatorname{Irr}(\mathbb{G})$ is an interpolation set of $\mathrm{M}_{L}\left(L^{p}(\mathbb{G})\right)$ with constant $K$;

(2) $p \neq \infty$ and $\mathbf{E} \subset \operatorname{Irr}(\mathbb{G})$ is an interpolation set of $\mathrm{M}_{R}\left(L^{p}(\mathbb{G})\right)$ with constant $K$;

(3) $\mathbf{E} \subset \operatorname{Irr}(\mathbb{G})$ is an interpolation set of $\mathrm{M}\left(L^{p}(\mathbb{G})\right)$ with constant $K$;

(4) there exist constants $K>0$ and $\theta, \theta^{\prime} \in \mathbb{R}$ with $\theta+\theta^{\prime}-\frac{1}{p}-\frac{1}{2} \neq 0$ such that for all $a \in c_{c}(\mathbf{E})$,

$$
\left\|m_{a}^{L} x\right\|_{p} \leq K\left\|Q^{\theta} a Q^{-\theta}\right\|_{\infty}\|x\|_{p}, \quad\left\|m_{a}^{R} x\right\|_{p} \leq K\left\|Q^{\theta^{\prime}} a Q^{-\theta^{\prime}}\right\|_{\infty}\|x\|_{p}, \quad x \in \operatorname{Pol}_{\mathbf{E}}(\mathbb{G}) .
$$

Then there exist constants $K_{1}, K_{2}>0$ such that for all $\pi \in \mathbf{E}$ and $1 \leq i, j, k, l \leq n_{\pi}$, we have

$$
\left\|u_{i j}^{(\pi)}\right\|_{p} \leq K_{1}\left\|u_{k l}^{(\pi)}\right\|_{p}
$$

and moreover

$$
\max \left\{\left\|Q_{\pi}\right\|,\left\|Q_{\pi}^{-1}\right\|\right\} \leq K_{2} .
$$

Proof. In the proof we always choose an appropriate basis of $\oplus_{\pi} H_{\pi}$ so that $Q_{\pi}$ is diagonal under this basis for $\pi \in \operatorname{Irr}(\mathbb{G})$. We first prove (4.1) and (4.2) under the assumption (4).

Assume that $K>0$ and $\theta, \theta^{\prime} \in \mathbb{R}$ with $\theta+\theta^{\prime}-\frac{1}{p}-\frac{1}{2} \neq 0$ such that for all $a \in c_{c}(\mathbf{E})$,

$$
\left\|m_{a}^{L} x\right\|_{p} \leq K\left\|Q^{\theta} a Q^{-\theta}\right\|_{\infty}\|x\|_{p}, \quad\left\|m_{a}^{R} x\right\|_{p} \leq K\left\|Q^{\theta^{\prime}} a Q^{-\theta^{\prime}}\right\|_{\infty}\|x\|_{p}, \quad x \in \operatorname{Pol}_{\mathbf{E}}(\mathbb{G}) .
$$

Let $\pi \in \mathbf{E}$ and $1 \leq i, j, k, l \leq n_{\pi}$. Then in particular we have

$$
\left\|m_{e_{k i}}^{L} x\right\|_{p} \leq K\left(Q_{\pi}\right)_{k k}^{\theta}\left(Q_{\pi}\right)_{i i}^{-\theta}\|x\|_{p}, \quad\left\|m_{e_{j l}}^{R} x\right\|_{p} \leq K\left(Q_{\pi}\right)_{j j}^{\theta^{\prime}}\left(Q_{\pi}\right)_{l l}^{-\theta^{\prime}}\|x\|_{p}, \quad x \in \operatorname{Pol}_{\mathbf{E}}(\mathbb{G}) .
$$

So by Proposition 2.2 we have

$$
\begin{aligned}
\left\|u_{i j}^{(\pi)}\right\|_{p} & =\left\|(\iota \otimes \operatorname{Tr})\left[\left(1 \otimes e_{j i}\right) u^{(\pi)}\right]\right\|_{p}=\left\|m_{e_{j l}}^{R} m_{e_{k i}}^{L}(\iota \otimes \operatorname{Tr})\left[\left(1 \otimes e_{l k}\right) u^{(\pi)}\right]\right\|_{p} \\
& \leq K^{2}\left(Q_{\pi}\right)_{k k}^{\theta}\left(Q_{\pi}\right)_{i i}^{-\theta}\left(Q_{\pi}\right)_{j j}^{\theta^{\prime}}\left(Q_{\pi}\right)_{l l}^{-\theta^{\prime}}\left\|(\iota \otimes \operatorname{Tr})\left[\left(1 \otimes e_{l k}\right) u^{(\pi)}\right]\right\|_{p} \\
& =K^{2}\left(Q_{\pi}\right)_{k k}^{\theta}\left(Q_{\pi}\right)_{i i}^{-\theta}\left(Q_{\pi}\right)_{j j}^{\theta^{\prime}}\left(Q_{\pi}\right)_{l l}^{-\theta^{\prime}}\left\|u_{k l}^{(\pi)}\right\|_{p} .
\end{aligned}
$$

In particular we get for any $1 \leq i, j \leq n_{\pi}$,

$$
K^{-2}\left(Q_{\pi}\right)_{j j}^{\theta+\theta^{\prime}}\left(Q_{\pi}\right)_{i i}^{-\theta-\theta^{\prime}}\left\|u_{j i}^{(\pi)}\right\|_{p} \leq\left\|u_{i j}^{(\pi)}\right\|_{p} \leq K^{2}\left(Q_{\pi}\right)_{j j}^{\theta+\theta^{\prime}}\left(Q_{\pi}\right)_{i i}^{-\theta-\theta^{\prime}}\left\|u_{j i}^{(\pi)}\right\|_{p}
$$

Recall that $Q_{\pi}$ is chosen diagonal, and note that by Lemma 2.4 and the formula (1.3),

$$
\left\|u_{i j}^{(\pi)}\right\|_{p}=\left\|\sigma_{-\mathrm{i} / p}\left(\left(u_{i j}^{(\pi)}\right)^{*}\right)\right\|_{p}=\left(Q_{\pi}\right)_{i i}^{-\frac{1}{p}}\left(Q_{\pi}\right)_{j j}^{-\frac{1}{p}}\left\|\left(u_{i j}^{(\pi)}\right)^{*}\right\|_{p}=\left(Q_{\pi}\right)_{i i}^{-\frac{1}{p}}\left(Q_{\pi}\right)_{j j}^{-\frac{1}{p}}\left\|S\left(u_{j i}^{(\pi)}\right)\right\|_{p} .
$$

But using the polar decomposition of $S$ in (1.4) and (1.5) and recalling that $Q_{\pi}$ is chosen diagonal, we have

$$
\left\|S\left(u_{j i}^{(\pi)}\right)\right\|_{p}=\left\|R\left(\tau_{-\frac{i}{2}}\left(u_{j i}^{(\pi)}\right)\right)\right\|_{p}=\left\|\tau_{-\frac{i}{2}}\left(u_{j i}^{(\pi)}\right)\right\|_{p}=\left(Q_{\pi}\right)_{j j}^{\frac{1}{2}}\left(Q_{\pi}\right)_{i i}^{-\frac{1}{2}}\left\|u_{j i}^{(\pi)}\right\|_{p} .
$$

The above three inequalities yield that

$$
\left(Q_{\pi}\right)_{i i}^{\theta+\theta^{\prime}-\frac{1}{p}-\frac{1}{2}}\left(Q_{\pi}\right)_{j j}^{-\theta-\theta^{\prime}-\frac{1}{p}+\frac{1}{2}} \leq K^{2}, \quad\left(Q_{\pi}\right)_{i i}^{-\theta-\theta^{\prime}-\frac{1}{p}+\frac{1}{2}}\left(Q_{\pi}\right)_{j j}^{\theta+\theta^{\prime}-\frac{1}{p}-\frac{1}{2}} \leq K^{2}
$$


Note that $i$ and $j$ are arbitrarily chosen and that $\left\|Q_{\pi}\right\| \geq 1,\left\|Q_{\pi}^{-1}\right\| \geq 1$, so the above inequalities yield

Combining this with (4.3), we also get

$$
\max \left\{\left\|Q_{\pi}\right\|,\left\|Q_{\pi}^{-1}\right\|\right\} \leq K^{2 /\left|\theta+\theta^{\prime}-\frac{1}{p}-\frac{1}{2}\right|}
$$

$$
\left\|u_{i j}^{(\pi)}\right\|_{p} \leq K^{2+2 /\left|\theta+\theta^{\prime}-\frac{1}{p}-\frac{1}{2}\right|}\left\|u_{k l}^{(\pi)}\right\|_{p}
$$

as desired.

Now we assume that $(1)$ holds, that is, $\mathbf{E} \subset \operatorname{Irr}(\mathbb{G})$ is an interpolation set of $\mathrm{M}_{L}\left(L^{p}(\mathbb{G})\right)$. Let $a \in c_{c}(\mathbf{E})$. Then $a$ extends to bounded left multipliers on $L^{p}(\mathbb{G})$, and hence by Lemma 2.5 we have

$$
\left\|m_{a}^{L} x\right\|_{p} \leq K\|a\|_{\infty}\|x\|_{p}, \quad\left\|m_{a}^{R} x\right\|_{p} \leq K\left\|Q^{\frac{1}{2}} a Q^{-\frac{1}{2}}\right\|_{\infty}\|x\|_{p}, \quad x \in \operatorname{Pol}_{\mathbf{E}}(\mathbb{G}) .
$$

Taking $\theta=0, \theta^{\prime}=1 / 2$ in (4), then we obtain the desired inequalities (4.1) and (4.2). And the proof under the assumption (2) follows from a similar argument.

The assumption (3) can be viewed as a particular case of (4) by taking $\theta=1 / p, \theta^{\prime}=0$. So we establish the proposition.

Combining this proposition with Lemma 2.5, we deduce the following observation.

Lemma 4.6. Let $\mathbf{E} \subset \operatorname{Irr}(\mathbb{G})$ be a subset. Let $1 \leq p<\infty$. Then $\mathbf{E}$ is an interpolation set of $\mathrm{M}_{L}\left(L^{p}(\mathbb{G})\right)$ if and only if it is an interpolation set of $\mathrm{M}_{R}\left(L^{p}(\mathbb{G})\right)$.

Now we are able to characterize the $\Lambda(p)$-sets via the interpolation sets of bounded multipliers for $2<p<\infty$.

Theorem 4.7. Let $\mathbf{E} \subset \operatorname{Irr}(\mathbb{G})$ be a subset. Assume $2<p<\infty$. The following assertions are equivalent:

(1) $\mathbf{E}$ is a $\Lambda(p)$-set;

(2) there exists a constant $K>0$ such that for all $a \in \ell^{\infty}(\mathbf{E})$,

$$
\left\|m_{a}^{L} x\right\|_{p} \leq K\|a\|_{\infty}\|x\|_{p}, \quad\left\|m_{a}^{R} x\right\|_{p} \leq K\|a\|_{\infty}\|x\|_{p}, \quad x \in \operatorname{Pol}_{\mathbf{E}}(\mathbb{G}) ;
$$

(3) $\mathbf{E}$ is an interpolation set of $\mathrm{M}_{L}\left(L^{p}(\mathbb{G})\right)$;

(4) $\mathbf{E}$ is an interpolation set of $\mathrm{M}_{R}\left(L^{p}(\mathbb{G})\right)$;

(5) $\mathbf{E}$ is an interpolation set of $\mathrm{M}\left(L^{p}(\mathbb{G})\right)$.

If additionally the subset $\mathbf{E}$ is symmetric in the sense that $\pi \in \mathbf{E}$ if and only if $\bar{\pi} \in \mathbf{E}$, then the above assertions are also equivalent to:

(6) there exists a constant $K>0$ such that for all $a \in \ell^{\infty}(\mathbf{E})$,

$$
\left\|m_{a}^{L} x\right\|_{p} \leq K\|a\|_{\infty}\|x\|_{p}, \quad x \in \operatorname{Pol}_{\mathbf{E}}(\mathbb{G}) ;
$$

(7) there exists a constant $K>0$ such that for all $a \in \ell^{\infty}(\mathbf{E})$,

$$
\left\|m_{a}^{R} x\right\|_{p} \leq K\|a\|_{\infty}\|x\|_{p}, \quad x \in \operatorname{Pol}_{\mathbf{E}}(\mathbb{G}) .
$$

Proof. Note that the equivalence $(3) \Leftrightarrow(4)$ has been already given in the previous lemma and the implication $(5) \Rightarrow(4)$ is trivial. Since (3) and (4) are equivalent, the implication $(3) \Rightarrow(2)$ is also obvious.

$(1) \Rightarrow(5)$. Assume that $\mathbf{E}$ is a $\Lambda(p)$-set with constant $K$. Then together with Lemma 2.3 , we see that for all $a \in \ell^{\infty}(\mathbf{E})$,

$$
\left\|m_{a}^{R} x\right\|_{p} \leq K\left\|m_{a}^{R} x\right\|_{2} \leq K\|a\|_{\infty}\|x\|_{2} \leq K\|a\|_{\infty}\|x\|_{p}, \quad x \in \operatorname{Pol}(\mathbb{G}),
$$

which yields that $m_{a}^{R}$ extends to a bounded operator on $L^{p}(\mathbb{G})$. So $\mathbf{E}$ is an interpolation set of $\mathrm{M}_{R}\left(L^{p}(\mathbb{G})\right)$, and in particular by Proposition 4.5 , we may find a constant $K^{\prime}$ such that

$$
\max \left\{\left\|Q_{\pi}\right\|,\left\|Q_{\pi}^{-1}\right\|\right\} \leq K^{\prime}, \quad \pi \in \mathbf{E} .
$$

Hence by Lemma 2.5, (4.5) also yields that for all $a \in \ell^{\infty}(\mathbf{E})$,

$$
\left\|m_{a}^{L} x\right\|_{p} \leq K\left\|m_{a}^{L} x\right\|_{2} \leq K K^{\prime}\|a\|_{\infty}\|x\|_{2} \leq K K^{\prime}\|a\|_{\infty}\|x\|_{p}, \quad x \in \operatorname{Pol}(\mathbb{G}) .
$$

As a result, both maps $m_{a}^{L}$ and $m_{a}^{R}$ for $a \in \ell^{\infty}(\mathbf{E})$ extend to bounded operators on $L^{p}(\mathbb{G})$, which means that $\mathbf{E}$ is an interpolation set of $\mathrm{M}\left(L^{p}(\mathbb{G})\right)$. 
$(2) \Rightarrow(1)$. Assume that (2) holds and we take $K>0$ to be the constant satisfying

$$
\left\|m_{a}^{L} x\right\|_{p} \leq K\|a\|_{\infty}\|x\|_{p}, \quad\left\|m_{a}^{R} x\right\|_{p} \leq K\|a\|_{\infty}\|x\|_{p}, \quad x \in \operatorname{Pol}_{\mathbf{E}}(\mathbb{G}), a \in \ell^{\infty}(\mathbf{E}) .
$$

For each $\pi \in \operatorname{Irr}(\mathbb{G})$, since the operator $Q_{\pi}$ is positive, we may fix a basis in $H_{\pi}$ such that the associated matrix of $Q_{\pi}$ is diagonal, and denote by $u^{(\pi)} \in \mathbb{M}_{n_{\pi}}(C(\mathbb{G}))$ the representation matrix under this basis.

Let us first show that for $\pi \in \operatorname{Irr}(\mathbb{G})$ and $1 \leq i \leq n_{\pi}$, we have some constant $C_{0}>0$ such that

$$
\left\|u_{i i}^{(\pi)}\right\|_{p}^{2} \leq C_{0} p\left\|u_{i i}^{(\pi)}\right\|_{2}^{2}=C_{0} p d_{\pi}^{-1}\left(Q_{\pi}^{-1}\right)_{i i} .
$$

Take the $n_{\pi} \times n_{\pi}$ matrices

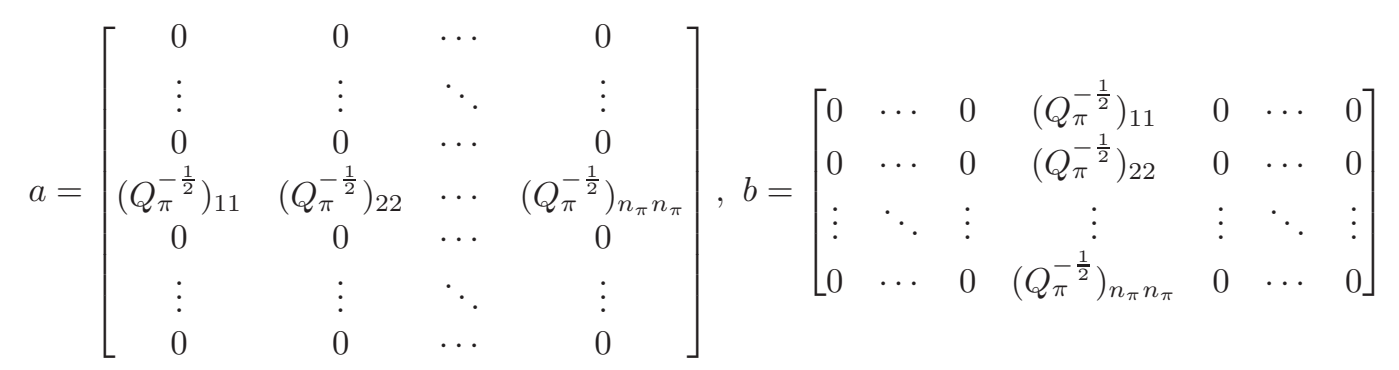

where the nonzero coefficients are in the $i$-th row of $a$, and in the $i$-th column of $b$. Note that $\|a\|_{\infty}=d_{\pi}^{1 / 2}$. Let $y=\sum_{j=1}^{n_{\pi}}\left(Q_{\pi}^{-\frac{1}{2}}\right)_{j j} u_{i j}^{(\pi)}$. Then by Proposition 2.2,

$$
y=(\iota \otimes \operatorname{Tr})\left[(1 \otimes b) u^{(\pi)}\right], \quad u_{i i}^{(\pi)}=d_{\pi}^{-1} m_{a}^{R} y .
$$

According to (4.6) we have

$$
\left\|u_{i i}^{(\pi)}\right\|_{p} \leq K d_{\pi}^{-\frac{1}{2}}\|y\|_{p}
$$

Let $\varepsilon_{j}^{(\pi)}\left(1 \leq j \leq n_{\pi}\right)$ be a sequence of independent Rademacher variables on a probability space $(\Omega, P)$ and write the $n_{\pi} \times n_{\pi}$ matrix

$$
\mathfrak{e}=\left[\begin{array}{cccc}
\varepsilon_{1}^{(\pi)} & 0 & \cdots & 0 \\
0 & \varepsilon_{2}^{(\pi)} & \cdots & 0 \\
\vdots & \vdots & \ddots & \vdots \\
0 & 0 & \cdots & \varepsilon_{n_{\pi}}^{(\pi)}
\end{array}\right]
$$

Again by (4.6) and by Theorem 1.7 we have with some universal constant $C>0$,

$$
\begin{aligned}
\|y\|_{p} & =\int_{\Omega}\left\|m_{\mathfrak{e} \mathfrak{e}}^{R} y\right\|_{p} d P \leq K \int_{\Omega}\left\|m_{\mathfrak{e}}^{R} y\right\|_{p} d P=K \int_{\Omega}\left\|\sum_{j=1}^{n_{\pi}}\left(Q_{\pi}^{-\frac{1}{2}}\right)_{j j} \varepsilon_{j}^{(\pi)} u_{i j}^{(\pi)}\right\|_{p} d P \\
& \leq C K \sqrt{p}\left\|\left(\left(Q_{\pi}^{-\frac{1}{2}}\right)_{j j} u_{i j}^{(\pi)} D^{\frac{1}{p}}\right)\right\|_{C R_{p}\left[L^{p}(\mathbb{G})\right]},
\end{aligned}
$$

where

$$
\begin{aligned}
& \left\|\left(\left(Q_{\pi}^{-\frac{1}{2}}\right)_{j j} u_{i j}^{(\pi)} D^{\frac{1}{p}}\right)\right\|_{C R_{p}\left[L^{p}(\mathbb{G})\right]} \\
= & \max \left\{\left\|\left(D^{\frac{1}{p}} \sum_{j=1}^{n_{\pi}}\left(Q_{\pi}^{-1}\right)_{j j}\left(u_{i j}^{(\pi)}\right)^{*} u_{i j}^{(\pi)} D^{\frac{1}{p}}\right)^{\frac{1}{2}}\right\|_{p, \mathrm{H}},\left\|\left(\sum_{j=1}^{n_{\pi}}\left(Q_{\pi}^{-1}\right)_{j j} u_{i j}^{(\pi)} D^{\frac{2}{p}}\left(u_{i j}^{(\pi)}\right)^{*}\right)^{\frac{1}{2}}\right\|_{p, \mathrm{H}}\right\} .
\end{aligned}
$$


Recall (1.6) and that the matrix $Q_{\pi}$ under the chosen basis is diagonal. We have

$$
\begin{aligned}
\sum_{j=1}^{n_{\pi}}\left(Q_{\pi}^{-1}\right)_{j j}\left(u_{i j}^{(\pi)}\right)^{*} u_{i j}^{(\pi)} & =\sum_{j=1}^{n_{\pi}}\left(Q_{\pi}^{-1}\right)_{j j} S\left(u_{j i}^{(\pi)}\right) u_{i j}^{(\pi)}=\sum_{j=1}^{n_{\pi}}\left(Q_{\pi}^{-1}\right)_{j j}\left(S^{-1} \circ S^{2}\right)\left(u_{j i}^{(\pi)}\right) u_{i j}^{(\pi)} \\
& =\sum_{j=1}^{n_{\pi}}\left(Q_{\pi}^{-1}\right)_{j j} S^{-1}\left(\left(Q_{\pi}\right)_{j j} u_{j i}^{(\pi)}\left(Q_{\pi}^{-1}\right)_{i i}\right) u_{i j}^{(\pi)} \\
& =S^{-1}\left(\sum_{j=1}^{n_{\pi}}\left(Q_{\pi}^{-1}\right)_{i i}\left(u_{j i}^{(\pi)}\right)^{*} u_{j i}^{(\pi)}\right)=\left(Q_{\pi}^{-1}\right)_{i i} S^{-1}(1)=\left(Q_{\pi}^{-1}\right)_{i i}
\end{aligned}
$$

where the last line above follows from the fact that $u^{(\pi)}$ is unitary. Recall Proposition $1.5(1)$ and (1.3). We then have

$$
u_{i j}^{(\pi)} D^{\frac{1}{p}}=D^{\frac{1}{p}} \sigma_{\frac{i}{p}}\left(u_{i j}^{(\pi)}\right)=D^{\frac{1}{p}}\left(Q_{\pi}^{-\frac{1}{p}}\right)_{i i} u_{i j}^{(\pi)}\left(Q_{\pi}^{-\frac{1}{p}}\right)_{j j},
$$

and hence

$$
D^{\frac{1}{p}}\left(u_{i j}^{(\pi)}\right)^{*}=\left(Q_{\pi}^{-\frac{1}{p}}\right)_{i i}\left(Q_{\pi}^{-\frac{1}{p}}\right)_{j j}\left(u_{i j}^{(\pi)}\right)^{*} D^{\frac{1}{p}}
$$

Therefore

$$
\sum_{j=1}^{n_{\pi}}\left(Q_{\pi}^{-1}\right)_{j j} u_{i j}^{(\pi)} D^{\frac{2}{p}}\left(u_{i j}^{(\pi)}\right)^{*}=D^{\frac{1}{p}} \sum_{j=1}^{n_{\pi}}\left(Q_{\pi}^{-\frac{2}{p}}\right)_{i i}\left(Q_{\pi}^{-\frac{2}{p}-1}\right)_{j j} u_{i j}^{(\pi)}\left(u_{i j}^{(\pi)}\right)^{*} D^{\frac{1}{p}}
$$

Recall that by Proposition 4.5 we have $K_{1}=\sup _{\pi \in \mathbf{E}}\left\|Q_{\pi}\right\|<\infty, K_{2}=\sup _{\pi \in \mathbf{E}}\left\|Q_{\pi}^{-1}\right\|<\infty$, so the above expression can be estimated as

$$
\sum_{j=1}^{n_{\pi}}\left(Q_{\pi}^{-1}\right)_{j j} u_{i j}^{(\pi)} D^{\frac{2}{p}}\left(u_{i j}^{(\pi)}\right)^{*} \leq K_{2}^{\frac{4}{p}+1} K_{1}\left(Q_{\pi}^{-1}\right)_{i i} D^{\frac{1}{p}} \sum_{j=1}^{n_{\pi}} u_{i j}^{(\pi)}\left(u_{i j}^{(\pi)}\right)^{*} D^{\frac{1}{p}}=K_{2}^{\frac{4}{p}+1} K_{1}\left(Q_{\pi}^{-1}\right)_{i i} D^{\frac{2}{p}}
$$

Together with (4.11) we deduce

$$
\left\|\left(\left(Q_{\pi}^{-\frac{1}{2}}\right)_{j j} u_{i j}^{(\pi)}\right)\right\|_{C R_{p}\left[L^{p}(\mathbb{G})\right]}^{2} \leq K_{2}^{\frac{4}{p}+1} K_{1}\left(Q_{\pi}^{-1}\right)_{i i} .
$$

Back to (4.10) we get $\|y\|_{p}^{2} \leq C^{2} K^{2} K_{2}^{\frac{4}{p}+1} K_{1} p\left(Q_{\pi}^{-1}\right)_{i i}$ and by (4.9) we obtain

$$
\left\|u_{i i}^{(\pi)}\right\|_{p}^{2} \leq C^{2} K^{4} K_{2}^{\frac{4}{p}+1} K_{1} p d_{\pi}^{-1}\left(Q_{\pi}^{-1}\right)_{i i}
$$

whence (4.7), as desired.

Now take $x \in \operatorname{Pol}_{\mathbf{E}}(\mathbb{G})$. Choose by polar decomposition and diagonalization two sequences of unitary matrices $v=\left(v_{\pi}\right)_{\pi \in \mathbf{E}}, v^{\prime}=\left(v_{\pi}^{\prime}\right)_{\pi \in \mathbf{E}} \in \ell^{\infty}(\mathbf{E})$ such that $c_{\pi}:=v_{\pi} v_{\pi}^{\prime} \hat{x}(\pi) Q_{\pi} v_{\pi}^{*}$ is a diagonal matrix for each $\pi$. Write

$$
c_{\pi}=\left[\begin{array}{cccc}
c_{11}^{(\pi)} & 0 & \cdots & 0 \\
0 & c_{22}^{(\pi)} & \cdots & 0 \\
\vdots & \ddots & \vdots & \vdots \\
0 & 0 & \cdots & c_{n_{\pi} n_{\pi}}^{(\pi)}
\end{array}\right], \quad c_{11}^{(\pi)}, \ldots, c_{n_{\pi} n_{\pi}}^{(\pi)} \in \mathbb{C}
$$


and denote $\mathfrak{e}=\left(\mathfrak{e}_{\pi}\right)_{\pi \in \mathbf{E}}$. By (4.6)-(4.7) and Theorem 1.7 we have with some universal constant $C>0$,

$$
\begin{aligned}
\|x\|_{p} & =\left\|m_{v^{\prime *} v^{*}}^{R} m_{v}^{L}\left(\sum_{\pi \in \mathbf{E}} d_{\pi}(\iota \otimes \operatorname{Tr})\left[\left(1 \otimes c_{\pi}\right) u^{(\pi)}\right]\right)\right\|_{p} \leq K^{2}\left\|\sum_{\pi \in \mathbf{E}} d_{\pi}(\iota \otimes \operatorname{Tr})\left[\left(1 \otimes c_{\pi}\right) u^{(\pi)}\right]\right\|_{p} \\
& \leq K^{3} \int_{\Omega}\left\|m_{\mathfrak{e}}^{R}\left(\sum_{\pi \in \mathbf{E}} d_{\pi}(\iota \otimes \operatorname{Tr})\left[\left(1 \otimes c_{\pi}\right) u^{(\pi)}\right]\right)\right\|_{p} d P=K^{3} \int_{\Omega}\left\|\sum_{\pi \in \mathbf{E}} d_{\pi} \sum_{j=1}^{n_{\pi}} \varepsilon_{j}^{(\pi)} c_{j j}^{(\pi)} u_{j j}^{(\pi)}\right\|_{p} d P \\
& \leq K^{3} C \sqrt{p}\left(\sum_{\pi \in \mathbf{E}} \sum_{j=1}^{n_{\pi}} d_{\pi}^{2}\left(c_{j j}^{(\pi)}\right)^{2}\left\|u_{j j}^{(\pi)}\right\|_{p}^{2}\right)^{\frac{1}{2}} \leq C_{0}^{\frac{1}{2}} C p K^{3}\left(\sum_{\pi \in \mathbf{E}} \sum_{j=1}^{n_{\pi}} d_{\pi}^{2}\left(c_{j j}^{(\pi)}\right)^{2}\left\|u_{j j}^{(\pi)}\right\|_{2}^{2}\right)^{\frac{1}{2}} \\
& =C_{0}^{\frac{1}{2}} C p K^{3}\left\|\sum_{\pi \in \mathbf{E}} \sum_{j=1}^{n_{\pi}} d_{\pi} c_{j j}^{(\pi)} u_{j j}^{(\pi)}\right\|_{2}
\end{aligned}
$$

where the last equality follows from the fact that $\left\{u_{i i}^{(\pi)}: 1 \leq i \leq n_{\pi}\right\}$ are orthogonal with respect to $h$ according to (1.2). Using Lemma 2.3 we have

$$
\left\|\sum_{\pi \in \mathbf{E}} \sum_{j=1}^{n_{\pi}} d_{\pi} c_{j j}^{(\pi)} u_{j j}^{(\pi)}\right\|_{2}=\left\|m_{v v^{\prime}}^{R} m_{v^{*}}^{L} x\right\|_{2} \leq K_{1} K_{2}\|x\|_{2} .
$$

Hence the above two inequalities together yield

$$
\|x\|_{p} \leq C_{0}^{\frac{1}{2}} C p K^{3} K_{1} K_{2}\|x\|_{2} .
$$

So we prove that $\mathbf{E}$ is a $\Lambda(p)$-set.

Finally let us discuss (6) and (7). In fact, if $\mathbf{E}$ is symmetric, we note that $X=\operatorname{Pol}_{\mathbf{E}}(\mathbb{G})$ satisfies the assumption of Lemma 2.5. So if (6) holds, by Lemma 2.5 we have for all $a \in \ell^{\infty}(\mathbf{E})$,

$$
\left\|m_{a}^{R} x\right\|_{p} \leq K\left\|Q^{1 / 2} a Q^{-1 / 2}\right\|_{\infty}\|x\|_{p}, \quad x \in \operatorname{Pol}_{\mathbf{E}}(\mathbb{G}) .
$$

and hence using Proposition 4.5 and the assumption in (6), we obtain the inequality

$$
\max \left\{\left\|Q_{\pi}\right\|,\left\|Q_{\pi}^{-1}\right\|\right\} \leq K^{\prime}, \quad \pi \in \mathbf{E}
$$

for some constant $K^{\prime}>0$. Thus coming back to (4.12) again, we see that the condition (7) holds as well. Conversely, we may also show in the same way that (7) implies (6). So in other words the assertions (6) and (7) are equivalent, and in particular they are equivalent to the assertion (2).

As mentioned previously, we conclude in the following that any Sidon set for a compact quantum group $\mathbb{G}$ is a $\Lambda(p)$-set for all $1<p<\infty$, thereby considerably improving the earlier work [BM13]. In fact as in the classical case, we have the stronger result below. Recall that in Theorem 3.3 we have some generalized notions of the Sidon set.

Theorem 4.8. Assume that $\mathbf{E} \subset \operatorname{Irr}(\mathbb{G})$ is an interpolation set of $\mathrm{M}\left(L^{\infty}(\mathbb{G})\right)$. Then $\mathbf{E}$ is a $\Lambda(p)$-set for all $1<p<\infty$.

Proof. Assume that $\mathbf{E}$ is an interpolation set of $\mathrm{M}\left(L^{\infty}(\mathbb{G})\right)$ with constant $K$. Let $a \in \ell^{\infty}(\mathbf{E})$. Then $a$ extends to a bounded multiplier $\tilde{a} \in \mathrm{M}\left(L^{\infty}(\mathbb{G})\right)$, and by Proposition 2.7,

$$
\left\|Q^{1 / 4} \tilde{a} Q^{-1 / 4}\right\|_{\infty} \leq\|\tilde{a}\|_{\mathrm{M}\left(L^{\infty}(\mathbb{G})\right)} \leq K\|a\|_{\infty} .
$$

Consider

$$
T_{z}=m_{a(z)}^{L}, \quad a_{\pi}^{(z)}=Q_{\pi}^{-\frac{z}{2}+\frac{1}{4}} \tilde{a}_{\pi} Q_{\pi}^{\frac{z}{2}-\frac{1}{4}}, \quad \pi \in \operatorname{Irr}(\mathbb{G}), z=t_{1}+\mathrm{i} t_{2}, 0 \leq t_{1} \leq 1, t_{2} \in \mathbb{R} .
$$

Observe that by Lemma 2.3 , the operators $m_{a^{(z)}}^{L}$ for $z=1+$ it are bounded on $L^{2}(\mathbb{G})$ with norm

$$
\left\|m_{a^{(z)}}^{L}\right\|_{B\left(L^{2}(\mathbb{G})\right)}=\left\|Q^{1 / 4} \tilde{a} Q^{-1 / 4}\right\|_{\infty} \leq K\|a\|_{\infty} .
$$

So by the Stein interpolation theorem (see e.g. [Lun09, Theorem 2.7]) for $2 \leq p<\infty$ we have $\left\|T_{2 / p} x\right\|_{p} \leq K_{0}\|a\|_{\infty}\|x\|_{p}$ for $x \in L^{p}(\mathbb{G})$. Let $K_{1}=\sup _{\pi \in \mathbf{E}}\left\|Q_{\pi}\right\|, K_{2}=\sup _{\pi \in \mathbf{E}}\left\|Q_{\pi}^{-1}\right\|$. Both 
$K_{1}$ and $K_{2}$ are finite by Proposition 4.5. Rewrite $b_{\pi}=Q_{\pi}^{-\frac{1}{p}+\frac{1}{4}} a_{\pi} Q_{\pi}^{\frac{1}{p}-\frac{1}{4}}$ and the above argument yields that for $b \in \ell^{\infty}(\mathbf{E})$ and $x \in \operatorname{Pol}_{\mathbf{E}}(\mathbb{G})$,

$$
\left\|m_{b}^{L} x\right\|_{p} \leq K\|a\|_{\infty}\|x\|_{p}=K_{0} \sup _{\pi \in \mathbf{E}}\left\|Q_{\pi}^{\frac{1}{p}-\frac{1}{4}} b_{\pi} Q_{\pi}^{-\frac{1}{p}+\frac{1}{4}}\right\|_{\infty}\|x\|_{p} \leq K_{0} K_{1}^{\frac{1}{p}-\frac{1}{4}} K_{2}^{\frac{1}{p}-\frac{1}{4}}\|b\|_{\infty}\|x\|_{p} .
$$

Similar argument also applies to $m_{a}^{R}$ (where the above $T_{z}$ should be simply taken as $m_{Q^{1 / 4}}^{R} \tilde{a} Q^{-1 / 4}$ identically). Now we may take $K>0$ to be the constant satisfying

$$
\left\|m_{a}^{L} x\right\|_{p} \leq K\|a\|_{\infty}\|x\|_{p}, \quad\left\|m_{a}^{R} x\right\|_{p} \leq K\|a\|_{\infty}\|x\|_{p}, \quad x \in \operatorname{Pol}_{\mathbf{E}}(\mathbb{G}), a \in \ell^{\infty}(\mathbf{E}) .
$$

Thus $\mathbf{E}$ is a $\Lambda(p)$-set for all $1<p<\infty$ according to Theorem 4.7.

By Theorem 3.15, we have the following corollary, as desired.

Corollary 4.9. If $\mathbf{E} \subset \operatorname{Irr}(\mathbb{G})$ is a Sidon set, then $\mathbf{E}$ is a $\Lambda(p)$-set for all $1<p<\infty$.

Below we give two more generalizations of the main result in [BM13]. For convenience we introduce the following definitions.

Definition 4.10. We say that $\mathbf{E} \subset \operatorname{Irr}(\mathbb{G})$ is a central interpolation set of $\mathrm{M}\left(L^{\infty}(\mathbb{G})\right)$ if for each bounded scalar sequence $\left(c_{\pi}\right)_{\pi \in \mathbf{E}} \subset \mathbb{C}$, there exists a bounded sequence $\left(\tilde{c}_{\pi}\right)_{\pi \in \operatorname{Irr}(\mathbb{G})} \subset \mathbb{C}$ with $\tilde{c}_{\pi}=c_{\pi}$ for $\pi \in \mathbf{E}$ such that $\tilde{c}=\left(\tilde{c}_{\pi} \operatorname{Id}_{\pi}\right)_{\pi \in \operatorname{Irr}(\mathbb{G})}$ is a bounded multiplier on $L^{\infty}(\mathbb{G})$.

We remark that if $\mathbf{E}$ is a Sidon set or an interpolation set of $\mathrm{M}\left(L^{\infty}(\mathbb{G})\right)$, or if $\mathbb{G}$ is of $\mathrm{Kac}$ type and $\mathbf{E}$ is a central Sidon set which will be introduced in Section 5 , then $\mathbf{E}$ is such a central interpolation set of $\mathrm{M}\left(L^{\infty}(\mathbb{G})\right)$.

Theorem 4.11. Let $\mathbb{G}$ be a compact quantum group. Assume that $\mathbf{E}$ is a central interpolation set of $\mathrm{M}\left(L^{\infty}(\mathbb{G})\right)$.

(1) If $1<p<\infty$ and $\sup _{\pi \in \mathbf{E}}\left\|\chi_{\pi}\right\|_{p}<\infty$, then $\mathbf{E}$ is a central $\Lambda(p)$-set;

(2) If $\sup _{\pi \in \mathbf{E}} d_{\pi}<\infty$, then $\mathbf{E}$ is a $\Lambda(p)$-set for $1<p<\infty$.

Proof. As is in the previous proof, for each $\pi \in \operatorname{Irr}(\mathbb{G})$, we fix a basis in $H_{\pi}$ such that the associated matrix of $Q_{\pi}$ is diagonal, and denote by $u^{(\pi)} \in \mathbb{M}_{n_{\pi}}(C(\mathbb{G}))$ the representation matrix under this basis.

Denote by $\left(\varepsilon_{\pi}\right)_{\pi \in \mathbf{E}}$ a Rademacher sequence on a probability space $(\Omega, P)$ and write $\mathfrak{e}=$ $\left(\varepsilon_{\pi} \operatorname{Id}_{\pi}\right)_{\pi \in \mathbf{E}} \in \ell^{\infty}(\mathbf{E})$. The same argument as in the proof of Theorem 4.8 yields that we may find $K>0$ such that for $x \in \operatorname{Pol}_{\mathbf{E}}(\mathbb{G}), \omega \in \Omega$ and $2<p<\infty$,

$$
\|x\|_{p}=\left\|m_{\mathfrak{e}(\omega)^{2}}^{R} x\right\|_{p} \leq K\left\|m_{\mathfrak{e}(\omega)}^{R} x\right\|_{p} .
$$

Integrating the inequality over $\omega \in \Omega$, we get

$$
\|x\|_{p} \leq K \int_{\Omega}\left\|\sum_{\pi \in \mathbf{E}} d_{\pi} \varepsilon_{\pi}(\iota \otimes \operatorname{Tr})\left[\left(1 \otimes \hat{x}(\pi) Q_{\pi}\right) u^{(\pi)}\right]\right\|_{p} d P .
$$

By Theorem 1.7, we have with some $K_{0}>0$,

$$
\begin{aligned}
& \int_{\Omega}\left\|\sum_{\pi \in \mathbf{E}} d_{\pi} \varepsilon_{\pi}(\iota \otimes \operatorname{Tr})\left[\left(1 \otimes \hat{x}(\pi) Q_{\pi}\right) u^{(\pi)}\right]\right\|_{p} d P \\
\leq & K_{0} \sqrt{p}\left(\sum_{\pi \in \mathbf{E}}\left\|d_{\pi}(\iota \otimes \operatorname{Tr})\left[\left(1 \otimes \hat{x}(\pi) Q_{\pi}\right) u^{(\pi)}\right]\right\|_{p}^{2}\right)^{1 / 2} .
\end{aligned}
$$

Therefore

$$
\|x\|_{p} \leq K K_{0} \sqrt{p}\left(\sum_{\pi \in \mathbf{E}}\left\|d_{\pi}(\iota \otimes \operatorname{Tr})\left[\left(1 \otimes \hat{x}(\pi) Q_{\pi}\right) u^{(\pi)}\right]\right\|_{p}^{2}\right)^{1 / 2} .
$$

(1) If $2<p<\infty$ and $x=\sum_{\pi \in \mathbf{E}} c_{\pi} \chi_{\pi} \in \operatorname{Pol}_{\mathbf{E}}(\mathbb{G})$ with $\left(c_{\pi}\right)_{\pi \in \mathbf{E}} \subset \mathbb{C}$, then the above inequality (4.13) reads

$$
\|x\|_{p} \leq K K_{0} \sqrt{p}\left(\sum_{\pi \in \mathbf{E}}\left|c_{\pi}\right|^{2}\left\|\chi_{\pi}\right\|_{p}^{2}\right)^{1 / 2} .
$$


Note that by (1.2) and the choice of basis in $H_{\pi}$, we have $\left\|\chi_{\pi}\right\|_{2}^{2}=h\left(\chi_{\pi}^{*} \chi_{\pi}\right)=1$. Also recall that $\chi_{\pi}$ are orthogonal with respect to $h$. Thus the condition $K_{1}=\sup _{\pi \in \mathbf{E}}\left\|\chi_{\pi}\right\|_{p}<\infty$ implies

$$
\|x\|_{p} \leq K K_{1} K_{0} \sqrt{p}\left(\sum_{\pi \in \mathbf{E}}\left|c_{\pi}\right|^{2}\left\|\chi_{\pi}\right\|_{2}^{2}\right)^{1 / 2}=\|x\|_{2} .
$$

Therefore $\mathbf{E}$ is a central $\Lambda(p)$-set.

(2) Assume $K_{2}=\sup _{\pi \in \mathbf{E}} d_{\pi}<\infty$. We need to show that the right term of the inequality (4.13) is not more than $\|x\|_{2}$, up to a constant independent of $x$. Note that by traciality of Tr, we have

$$
(\iota \otimes \operatorname{Tr})\left[\left(1 \otimes \hat{x}(\pi) Q_{\pi}\right) u^{(\pi)}\right]=(\iota \otimes \operatorname{Tr})\left[u^{(\pi)}\left(1 \otimes \hat{x}(\pi) Q_{\pi}\right)\right]
$$

Also, we note that the $\operatorname{map} d_{\pi}^{-1}\left(\iota \otimes \operatorname{Tr}\left(\cdot\left(1 \otimes Q_{\pi}\right)\right)\right)$ is unital completely positive on $C(\mathbb{G}) \otimes B\left(H_{\pi}\right)$ for each $\pi \in \operatorname{Irr}(\mathbb{G})$, so by the Cauchy-Schwarz inequality,

$$
\begin{aligned}
& \left|d_{\pi}^{-1}(\iota \otimes \operatorname{Tr})\left[u^{(\pi)}\left(1 \otimes \hat{x}(\pi) Q_{\pi}\right)\right] D^{1 / p}\right|^{2} \\
= & D^{1 / p}\left|d_{\pi}^{-1}(\iota \otimes \operatorname{Tr})\left[u^{(\pi)}\left(1 \otimes \hat{x}(\pi) Q_{\pi}\right)\right]\right|^{2} D^{1 / p} \\
\leq & D^{1 / p} d_{\pi}^{-1}\left(\iota \otimes \operatorname{Tr}\left(\cdot Q_{\pi}\right)\right)\left[\left(1 \otimes \hat{x}(\pi)^{*}\right)\left(u^{(\pi)}\right)^{*} u^{(\pi)}(1 \otimes \hat{x}(\pi))\right] D^{1 / p} \\
= & d_{\pi}^{-1} D^{1 / p} \operatorname{Tr}\left(|\hat{x}(\pi)|^{2} Q_{\pi}\right) D^{1 / p}=d_{\pi}^{-1} \operatorname{Tr}\left(|\hat{x}(\pi)|^{2} Q_{\pi}\right) D^{2 / p} .
\end{aligned}
$$

Hence together with Proposition 1.5 (5),

$$
\begin{aligned}
& \sum_{\pi \in \mathbf{E}}\left\|d_{\pi}(\iota \otimes \operatorname{Tr})\left[\left(1 \otimes \hat{x}(\pi) Q_{\pi}\right) u^{(\pi)}\right]\right\|_{p}^{2}=\sum_{\pi \in \mathbf{E}}\left\|\left|d_{\pi}(\iota \otimes \operatorname{Tr})\left[\left(1 \otimes \hat{x}(\pi) Q_{\pi}\right) u^{(\pi)}\right] D^{1 / p}\right|^{2}\right\|_{p / 2, \mathrm{H}} \\
\leq & K_{2}^{2} \sum_{\pi \in \mathbf{E}} d_{\pi}^{2}\left\|\left|d_{\pi}^{-1}(\iota \otimes \operatorname{Tr})\left[\left(1 \otimes \hat{x}(\pi) Q_{\pi}\right) u^{(\pi)}\right] D^{1 / p}\right|^{2}\right\|_{p / 2, \mathrm{H}} \\
\leq & K_{2}^{2} \sum_{\pi \in \mathbf{E}} d_{\pi}^{2}\left\|d_{\pi}^{-1} \operatorname{Tr}\left(|\hat{x}(\pi)|^{2} Q_{\pi}\right) D^{2 / p}\right\|_{p / 2, \mathrm{H}}=\sum_{\pi \in \mathbf{E}} d_{\pi} \operatorname{Tr}\left(|\hat{x}(\pi)|^{2} Q_{\pi}\right)=\|\hat{x}\|_{2}^{2}=\|x\|_{2}^{2} .
\end{aligned}
$$

Now back to (4.13) we get $\|x\|_{p} \leq K K_{0} K_{2} \sqrt{p}\|x\|_{2}$, as desired.

Remark 4.12. Let us make a few remarks on the constant of $\Lambda(p)$-sets. In the proof of Theorem 4.7 and Theorem 4.8, we have shown that, if $\mathbf{E} \subset \operatorname{Irr}(\mathbb{G})$ is an interpolation set of $\mathrm{M}\left(L^{p}(\mathbb{G})\right)$ with constant $K$ for some $1<p<\infty$, then $\mathbf{E}$ is a $\Lambda(p)$-set with constant $c_{1} K^{c_{2}} p$ with two universal constants $c_{1}, c_{2}>0$; and if $\mathbf{E} \subset \operatorname{Irr}(\mathbb{G})$ is an interpolation set of $\mathrm{M}\left(L^{\infty}(\mathbb{G})\right)$, then $\mathbf{E}$ is a $\Lambda(p)$-set with constant $c_{1}^{\prime} K^{c_{2}^{\prime}} p$ with two universal constants $c_{1}^{\prime}, c_{2}^{\prime}>0$ for all $1<p<\infty$. It seems that these constants should be improved. Indeed, it is well-known that if $\mathbb{G}$ is a compact group or the dual quantum group of a discrete group, the constants above can be improved to $c_{1} K^{c_{2}} \sqrt{p}$ and $c_{1}^{\prime} K^{c_{2}^{\prime}} \sqrt{p}$ respectively (see [HR70, Har99]). On the other hand, we see that, for some subclass of Sidon sets as in Theorem 4.11, we may also obtain the constant in the form $c_{1} K^{c_{2}} \sqrt{p}$.

Example 4.13. (1) Following the notation in Example 3.17, we consider $\mathbb{G}=\prod_{k \geq 1} \mathbb{G}_{k}, \mathbb{G}_{k}=U_{N_{k}}^{+}$ and $\mathbf{E}=\left\{u^{(k)}: k \geq 1\right\} \subset \operatorname{Irr}(\mathbb{G})$. We saw in Example 3.17 that $\mathbf{E}$ is an interpolation set of $\mathrm{M}\left(L^{\infty}(\mathbb{G})\right)$, and hence by Theorem $4.8 \mathbf{E}$ is a $\Lambda(p)$-set for all $1<p<\infty$. Alternatively, recall the Haagerup type inequality shown in Brannan [Bra12, Theorem 6.3]: for $k \geq 1, x_{i j} \in \mathbb{C}$, $1 \leq i, j \leq N_{k}$

$$
\left\|\sum_{i, j=1}^{N_{k}} x_{i j} u_{i j}^{(k)}\right\|_{p} \leq C\left\|\sum_{i, j=1}^{N_{k}} x_{i j} u_{i j}^{(k)}\right\|_{2}
$$

for a universal constant $C$. So by Theorem 1.7 and the standard argument as in the beginning of the proof of Theorem 4.11, we have the following Khintchine type inequality: for a universal 
constant $K$ and for all $2 \leq p<\infty$ and all finitely supported sequences $\left(A_{k}\right) \in \prod_{k} \mathbb{M}_{N_{k}}$,

$$
\begin{aligned}
\left\|\sum_{k \geq 1} N_{k}(\iota \otimes \operatorname{Tr})\left[\left(1 \otimes A_{k}\right) u^{(k)}\right]\right\|_{L^{p}(\mathbb{G})} & \leq K \sqrt{p}\left(\sum_{k}\left\|N_{k}(\iota \otimes \operatorname{Tr})\left[\left(1 \otimes A_{k}\right) u^{(k)}\right]\right\|_{L^{p}(\mathbb{G})}^{2}\right)^{1 / 2} \\
& \leq C K \sqrt{p}\left(\sum_{k} N_{k} \operatorname{Tr}\left(\left|A_{k}\right|^{2}\right)\right)^{1 / 2} .
\end{aligned}
$$

If $n_{k}=1$ for all $k$, then the above inequality reduces to the classical Khintchine inequalities.

(2) Follow the notation in Example 3.18. We consider the sequence $\left(q_{n}\right)_{n>1} \subset[q, 1]$ with $q:=\inf _{n} q_{n}>0$ and the associated quantum group $\mathbb{G}=\prod_{n>1} \mathrm{SU}_{q_{n}}(2)$. In Example 3.18 we proved that $\mathbf{E}=\left\{u_{n}: n \geq 1\right\} \subset \operatorname{Irr}(\mathbb{G})$ is a Sidon set for $\mathbb{G}$. Note that the associated matrix $Q_{n}:=Q_{u_{n}}$ is diagonal with entries $\left\{q_{n}^{-1}, q_{n}\right\}$ under the standard basis and hence $\sup _{n} \operatorname{dim}_{q}\left(u_{n}\right)=$ $\sup _{n}\left(q_{n}+q_{n}^{-1}\right) \leq 1+q^{-1}<\infty$. So by Theorem 4.11 , $\mathbf{E}$ is also a $\Lambda(p)$-set for $2 \leq p<\infty$ and we obtain the following Khintchine type inequalities: there exists a constant $K>0$ (depending on $q$ ) so that for all finitely supported sequences $\left(A_{n}\right) \in \prod_{n \geq 1} \mathbb{M}_{2}$,

$$
\left(\sum_{n \geq 1} d_{n} \operatorname{Tr}\left(\left|A_{n}\right|^{2} Q_{n}\right)\right)^{1 / 2} \leq\left\|\sum_{n \geq 1} d_{n}(\iota \otimes \operatorname{Tr})\left[\left(1 \otimes A_{n} Q_{n}\right) u_{n}\right]\right\|_{L^{p}(\mathbb{G})} \leq K \sqrt{p}\left(\sum_{n \geq 1} d_{n} \operatorname{Tr}\left(\left|A_{n}\right|^{2} Q_{n}\right)\right)^{1 / 2}
$$

where $d_{n}=q_{n}+q_{n}^{-1}$. Note that by Proposition 4.5 and Theorem 4.7 , if $q=0$ and $q_{n} \rightarrow 0$, the subset $\mathbf{E}$ defined as above cannot be a $\Lambda(p)$-set for any $2<p<\infty$.

4.2. Independence of the interpolation parameters for $L_{(\theta)}^{p}(\mathbb{G})$. In this subsection we would like to show that our definition of $\Lambda(p)$-sets does not depend on different interpolation parameters of $L^{p}$-spaces associated to a compact quantum group $\mathbb{G}$. Recall that in Section 1.2, we introduce the space $L^{p}(\mathbb{G})$ as the complex interpolation space $\left(L^{\infty}(\mathbb{G}), L^{\infty}(\mathbb{G})_{*}\right)_{1 / p}$ associated to the compatible couple $\left(L^{\infty}(\mathbb{G}), L^{\infty}(\mathbb{G})_{*}\right)$ given by the embedding

$$
L^{\infty}(\mathbb{G}) \hookrightarrow L^{\infty}(\mathbb{G})_{*}, \quad x \mapsto h(\cdot x), \quad x \in L^{\infty}(\mathbb{G}) .
$$

However in [Kos84] Kosaki provides some other possibilities to define the complex interpolation scale $\left(L^{p}(\mathbb{G})\right)_{1 \leq p \leq \infty}$. More precisely, fix a parameter $0 \leq \theta \leq 1$, we may consider the compatible couple $\left(L^{\infty}(\mathbb{G}), L^{\infty}(\mathbb{G})_{*}\right)^{(\theta)}$ given by the embedding

$$
L^{\infty}(\mathbb{G}) \hookrightarrow L^{\infty}(\mathbb{G})_{*}, \quad x \mapsto h\left(\cdot \sigma_{-\theta \mathrm{i}}(x)\right), \quad x \in L^{\infty}(\mathbb{G}),
$$

and we define the complex interpolation space

$$
L_{(\theta)}^{p}(\mathbb{G})=\left(L^{\infty}(\mathbb{G}), L^{\infty}(\mathbb{G})_{*}\right)_{1 / p}^{(\theta)} .
$$

Note that $L_{(0)}^{p}(\mathbb{G})$ coincides with the space $L^{p}(\mathbb{G})$ defined before. In the language of Haagerup's $L^{p}$-spaces, we have considered the embedding

$$
L^{\infty}(\mathbb{G}) \hookrightarrow L^{1, \mathrm{H}}(\mathbb{G}), \quad x \mapsto D^{\theta} x D^{1-\theta}=\sigma_{-\theta \mathrm{i}}(x) D, \quad x \in L^{\infty}(\mathbb{G}),
$$

and for each $1 \leq p \leq \infty$ we have the isometric isomorphism

$$
L_{(\theta)}^{p}(\mathbb{G}) \rightarrow L^{p, \mathrm{H}}(\mathbb{G}), \quad x \mapsto D^{\frac{\theta}{p}} x D^{\frac{1-\theta}{p}}, \quad x \in L^{\infty}(\mathbb{G}) .
$$

In particular,

$$
\|x\|_{L_{(\theta)}^{p}(\mathbb{G})}=\left\|\sigma_{-\mathrm{i} \frac{\theta}{p}}(x) D^{\frac{1}{p}}\right\|_{L^{p, \mathrm{H}(\mathbb{G})}}=\left\|\sigma_{-\mathrm{i} \frac{\theta}{p}}(x)\right\|_{L_{(0)}^{p}(\mathbb{G})}, \quad x \in \operatorname{Pol}(\mathbb{G}) .
$$

The spaces $L_{(\theta)}^{p}(\mathbb{G})$ and $L_{\left(\theta^{\prime}\right)}^{p}(\mathbb{G})$ for different parameters $\theta, \theta^{\prime}$ are isometric as Banach spaces, but one needs to be careful with the parameter $\theta$ when doing the Fourier analysis on $\mathbb{G}$, for which we refer to [Cas13, Section 7] for some related discussions. So returning back to the topic on $\Lambda(p)$-sets, it is natural to ask if the notion of $\Lambda(p)$-sets is independent of the choice of the parameter $\theta$. In the following we give an affirmative answer. 
Proposition 4.14. Let $\mathbb{G}$ be a compact quantum group and let $2<p<\infty, 0 \leq \theta \leq 1$. Then $\mathbf{E} \subset \operatorname{Irr}(\mathbb{G})$ is a $\Lambda(p)$-set for $L_{(\theta)}^{p}(\mathbb{G})$, that is, there exists a constant $K>0$ with

$$
\|x\|_{L_{(\theta)}^{p}(\mathbb{G})} \leq K\|x\|_{L_{(\theta)}^{2}(\mathbb{G})}, \quad x \in \operatorname{Pol}_{\mathbf{E}}(\mathbb{G}),
$$

if and only if it is a $\Lambda(p)$-set for $L_{(0)}^{p}(\mathbb{G})$ in the sense of Definition 4.1.

Proof. Assume that $\mathbf{E} \subset \operatorname{Irr}(\mathbb{G})$ is a $\Lambda(p)$-set for $L_{(\theta)}^{p}(\mathbb{G})$ with the constant $K$ given above. Then for all $a \in \ell^{\infty}(\mathbf{E})$, together with the equality (4.14) we have for $x \in \operatorname{Pol}_{\mathbf{E}}(\mathbb{G})$,

$$
\left\|m_{a}^{R} x\right\|_{L_{(\theta)}^{p}(\mathbb{G})} \leq K\left\|m_{a}^{R} x\right\|_{L_{(\theta)}^{2}(\mathbb{G})}=K\left\|\sigma_{-\mathrm{i} \frac{\theta}{2}}\left(m_{a}^{R} x\right)\right\|_{L_{(0)}^{2}(\mathbb{G})} .
$$

By Lemma 2.6, we know that

$$
\mathcal{F}\left(\sigma_{-\mathrm{i} \frac{\theta}{2}}\left(m_{a}^{R} x\right)\right)=Q^{\frac{\theta}{2}} a \hat{x} Q^{\frac{\theta}{2}}
$$

Hence by Proposition 2.2,

$$
\left\|\sigma_{-\mathrm{i} \frac{\theta}{2}}\left(m_{a}^{R} x\right)\right\|_{L_{(0)}^{2}(\mathbb{G})}^{2}=\sum_{\pi \in \operatorname{Irr}(\mathbb{G})} d_{\pi} \operatorname{Tr}\left(\left|Q_{\pi}^{\frac{\theta}{2}} a \hat{x}(\pi) Q_{\pi}^{\frac{\theta}{2}}\right|^{2} Q_{\pi}\right) .
$$

Let $\pi \in \mathbf{E}$ and without loss of generality we choose an appropriate basis of $H_{\pi}$ so that $Q_{\pi}$ is diagonal under this basis. Assume that $a \in c_{c}(\mathbf{E})$ and take $x \in \operatorname{Pol}_{\mathbf{E}}(\mathbb{G})$, then the above inequalities gives

$$
\begin{aligned}
\left\|m_{a}^{R} x\right\|_{L_{(\theta)}^{p}(\mathbb{G})}^{2} & \leq K^{2}\left\|Q^{\frac{\theta}{2}} a Q^{-\frac{\theta}{2}}\right\|_{\infty}^{2} \sum_{\pi \in \mathbf{E}} d_{\pi} \operatorname{Tr}\left(\left|Q_{\pi}^{\frac{\theta}{2}} \hat{x}(\pi) Q_{\pi}^{\frac{\theta}{2}}\right|^{2} Q_{\pi}\right) \\
& =K^{2}\left\|Q^{\frac{\theta}{2}} a Q^{-\frac{\theta}{2}}\right\|_{\infty}^{2}\left\|\sigma_{-i \frac{\theta}{2}}(x)\right\|_{L_{(0)}^{2}(\mathbb{G})}^{2}=K^{2}\left\|Q^{\frac{\theta}{2}} a Q^{-\frac{\theta}{2}}\right\|_{\infty}^{2}\|x\|_{L_{(\theta)}^{2}(\mathbb{G})}^{2} \\
& \leq K^{2}\left\|Q^{\frac{\theta}{2}} a Q^{-\frac{\theta}{2}}\right\|_{\infty}^{2}\|x\|_{L_{(\theta)}^{p}(\mathbb{G})}^{2} .
\end{aligned}
$$

And a similar inequality can be proved for the map $m_{a}^{L}$. Then following the idea in the proof of Proposition 4.5, we may find a constant $K^{\prime}>0$ such that

$$
\max \left\{\left\|Q_{\pi}\right\|,\left\|Q_{\pi}^{-1}\right\|\right\} \leq K^{\prime}, \quad \pi \in \mathbf{E} .
$$

Therefore together with (4.14), Lemma 2.6 and Lemma 2.3, we get for all $x \in \operatorname{Pol}_{\mathbf{E}}(\mathbb{G})$,

$$
\begin{aligned}
\|x\|_{L_{(0)}^{p}(\mathbb{G})} & =\left\|\sigma_{\mathrm{i} \frac{\theta}{p}}(x)\right\|_{L_{(\theta)}^{p}(\mathbb{G})} \leq K\left\|\sigma_{\mathrm{i} \frac{\theta}{p}}(x)\right\|_{L_{(\theta)}^{2}(\mathbb{G})}=K\left\|\sigma_{\mathrm{i}\left(\frac{1}{p}-\frac{1}{2}\right) \theta}(x)\right\|_{L_{(0)}^{2}(\mathbb{G})} \\
& =K\left\|\mathcal{F}\left(\sigma_{\mathrm{i}\left(\frac{1}{p}-\frac{1}{2}\right) \theta}(x)\right)\right\|_{\ell^{2}(\hat{\mathbb{G}})}=K\left\|Q^{\frac{1}{2}-\frac{1}{p}} \hat{x} Q^{\frac{1}{2}-\frac{1}{p}}\right\|_{\ell^{2}(\hat{\mathbb{G}})} \\
& =K\left\|m_{Q^{\frac{1}{2}-\frac{1}{p}}}^{R} m_{Q^{\frac{1}{2}-\frac{1}{p}}}^{L}\right\|_{L_{(0)}^{2}(\mathbb{G})} \leq K\left(K^{\prime}\right)^{1-\frac{2}{p}}\|x\|_{L_{(0)}^{2}(\mathbb{G})} .
\end{aligned}
$$

So $\mathbf{E}$ is a $\Lambda(p)$-sets for $L_{(0)}^{p}(\mathbb{G})$.

Note that if conversely $\mathbf{E}$ is a $\Lambda(p)$-set for $L_{(0)}^{p}(\mathbb{G})$, then we have already shown in Proposition 4.5 that there exists a constant $K^{\prime}>0$ such that

$$
\max \left\{\left\|Q_{\pi}\right\|,\left\|Q_{\pi}^{-1}\right\|\right\} \leq K^{\prime}, \quad \pi \in \mathbf{E}
$$

So a similar estimation as above yields that $\mathbf{E}$ must be a $\Lambda(p)$-sets for $L_{(\theta)}^{p}(\mathbb{G})$. Therefore the proof is complete.

Finally we remark that all the discussions in the previous sections can be in fact reproduced for $L_{(\theta)}^{p}(\mathbb{G})$ with the similar idea, and we omit the details. 
4.3. Existence of $\Lambda(p)$-sets. In this short subsection we discuss the existence of $\Lambda(p)$-sets for compact quantum groups. We refer to the following result proved in the appendix: let $M$ be a von Neumann algebra with a normal faithful state $\varphi$ and $B=\left\{x_{k}: k \geq 1\right\} \subset M$ be an orthonormal system with respect to $\varphi$ such that $\sup _{k}\left\|x_{k}\right\|_{\infty}<\infty$, then there exists an infinite subset $Y \subset B$ and a constant $C_{p}$ such that $\|x\|_{p} \leq C_{p}\|x\|_{2}$ for all $x \in \operatorname{span}(Y)$. Immediately we deduce the existence of $\Lambda(p)$-sets with uniform dimension assumptions.

Theorem 4.15. Let $\mathbb{G}$ be a compact quantum group. Let $\mathbf{E} \subset \operatorname{Irr}(\mathbb{G})$ be an infinite subset with $\sup _{\pi \in \mathbf{E}} d_{\pi}<\infty$. Then for each $2<p<\infty$, there exists an infinite subset $\mathbf{F} \subset \mathbf{E}$ which is a $\Lambda(p)$-set for $\mathbb{G}$.

Proof. Denote $D_{0}=\sup _{\pi \in \mathbf{E}} d_{\pi}<\infty$ and fix $2 \leq p<\infty$. Then also $n_{\pi}=\operatorname{dim}\left(H_{\pi}\right) \leq d_{\pi} \leq D_{0}$. Choose an appropriate basis of $H_{\pi}$ such that the matrix $Q_{\pi}$ is diagonal under this basis. For each $\pi \in \mathbf{E}, 1 \leq i, j \leq n_{\pi}$, write $v_{i j}^{(\pi)}=\left\|u_{i j}^{(\pi)}\right\|_{2}^{-1} u_{i j}^{(\pi)}$. By $(1.2),\left\|v_{i j}^{(\pi)}\right\|_{\infty} \leq\left\|u_{i j}^{(\pi)}\right\|_{2}^{-1} \leq d_{\pi}^{2} \leq D_{0}^{2}$. Consider $B_{0}^{(\pi)}=\left\{v_{i j}^{(\pi)}: 1 \leq i, j \leq n_{\pi}\right\}$ for $\pi \in \mathbf{E}$, and $B_{0}=\cup_{\pi \in \mathbf{E}} B_{0}^{(\pi)}$. Then $B_{0}$ is an orthogonal system since $Q_{\pi}$ is chosen diagonal. Write $\mathbf{E}_{0}=\mathbf{E}$. According to the theorem in the appendix, we can find an infinite subset $B_{1} \subset B_{0}$ with constant $C_{1}>0$ such that for all finitely many $c_{1}, \ldots, c_{n} \subset \mathbb{C}, x_{1}, \ldots, x_{n} \subset B_{1}$,

$$
\left\|\sum_{l=1}^{n} c_{l} x_{l}\right\|_{p} \leq C_{1}\left\|\sum_{l=1}^{n} c_{l} x_{l}\right\|_{2} .
$$

Set $\mathbf{E}_{1}=\left\{\pi \in \mathbf{E}_{0}: \exists v_{i j}^{(\pi)} \in B_{1}\right\}$ and let $B_{1}^{(\pi)}=\left\{v_{i j}^{(\pi)} \in B_{1}: 1 \leq i, j \leq n_{\pi}\right\}$ for $\pi \in \mathbf{E}_{1}$. The last set is non-empty. Then

$$
B_{1}=\cup_{\pi \in \mathbf{E}_{1}} B_{1}^{(\pi)}, \quad \operatorname{Card}\left(B_{0}^{(\pi)} \backslash B_{1}^{(\pi)}\right) \leq n_{\pi}^{2}-1 \leq D_{0}^{2}-1, \pi \in \mathbf{E}_{1} .
$$

Repeating inductively the above procedures, if $k \geq 1$ and if $\exists \pi \in \mathbf{E}_{k}, B_{0}^{(\pi)} \backslash\left(\cup_{l=1}^{k} B_{l}^{(\pi)}\right) \neq \emptyset$, we construct the proper subsets $\mathbf{E}_{k+1} \subset \mathbf{E}_{k}, B_{k+1}^{(\pi)} \subset B_{0}^{(\pi)} \backslash\left(\cup_{l=1}^{k} B_{l}^{(\pi)}\right), B_{k+1}=\cup_{\pi \in \mathbf{E}_{k+1}} B_{k+1}^{(\pi)} \subset$ $B_{0} \backslash\left(\cup_{l=1}^{k} B_{l}\right)$ and a constant $C_{k+1}>0$ such that for all finitely many $c_{1}, \ldots, c_{n} \subset \mathbb{C}, x_{1}, \ldots, x_{n} \subset$ $B_{k+1}$

$$
\left\|\sum_{l=1}^{n} c_{l} x_{l}\right\|_{p} \leq C_{k+1}\left\|\sum_{l=1}^{n} c_{l} x_{l}\right\|_{2}
$$

and such that

$$
\operatorname{Card}\left(\mathbf{E}_{k+1}\right)=\infty, \quad \operatorname{Card}\left(B_{0}^{(\pi)} \backslash\left(\cup_{l=1}^{k+1} B_{l}^{(\pi)}\right) \leq D_{0}^{2}-(k+1), \pi \in \mathbf{E}_{k+1} .\right.
$$

Since $D_{0}$ is finite, the above inequality shows that there exists $\tilde{k} \leq D_{0}^{2}$ such that $B_{0}^{(\pi)}=\cup_{l=1}^{\tilde{k}} B_{l}^{(\pi)}$ for $\pi \in \mathbf{E}_{\tilde{k}}$. Let $\mathbf{F}=\mathbf{E}_{\tilde{k}}$ and $\tilde{B}_{l}=\cup_{\pi \in \mathbf{F}} B_{l}^{(\pi)} \subset B_{l}$. Then for each $1 \leq l \leq \tilde{k}$,

$$
\|x\|_{p} \leq C_{l}\|x\|_{2}, \quad x \in \operatorname{span}\left(\tilde{B}_{l}\right) .
$$

Since $\cup_{l=1}^{\tilde{k}} \tilde{B}_{l} \subset B_{0}$ is an orthonormal system and

$$
\operatorname{Pol}_{\mathbf{F}}(\mathbb{G})=\operatorname{span}\left(\cup_{\pi \in \mathbf{F}} B_{0}^{(\pi)}\right)=\operatorname{span}\left(\cup_{\pi \in \mathbf{F}} \cup_{l=1}^{\tilde{k}} B_{l}^{(\pi)}\right)=\operatorname{span}\left(\cup_{l=1}^{\tilde{k}} \tilde{B}_{l}\right),
$$

we obtain

$$
\|x\|_{p} \leq D_{0}^{2} \max \left\{C_{l}: 1 \leq l \leq \tilde{k}\right\}\|x\|_{2}, \quad x \in \operatorname{Pol}_{\mathbf{F}}(\mathbb{G}) .
$$

Hence $\mathbf{F} \subset \operatorname{Irr}(\mathbb{G})$ is the desired infinite $\Lambda(p)$-set.

The existence of $\Lambda(p)$-sets without the assumption $\sup _{\pi \in \mathbf{E}} d_{\pi}<\infty$ in the above theorem is in general not true, which can be seen from the non-existence of central $\Lambda(4)$-sets for the classical $\mathrm{SU}(2)$, as well as from the quantum non-tracial example 4.13.(2). 
4.4. Remarks on the lacunarity for $S U_{q}(2)$. In [HR70], a classical version of Theorem 4.8 was used to prove the fact that the special unitary group $\mathrm{SU}(2)$ does not admit any infinite Sidon set. The key observation therein is that $\mathrm{SU}(2)$ does not admit any infinite central $\Lambda(4)$-set. Here we want to show that, in strong contrast, the $q$-deformed quantum group $\mathrm{SU}_{q}(2)$ with $0<q<1$ does admit an infinite central $\Lambda(4)$-set. The non-traciality of the Haar state on $\mathrm{SU}_{q}(2)$ plays an essential role for this result. We recall that $\operatorname{Irr}\left(\mathrm{SU}_{q}(2)\right)$ can be identified with $\mathbb{N} \cup\{0\}$ and follow the notation in the preliminary part.

Proposition 4.16. Let $0<q<1$ and let $\mathbf{E}=\left\{n_{k} \in \mathbb{N} \cup\{0\}: k \geq 0\right\} \subset \operatorname{Irr}\left(\operatorname{SU}_{q}(2)\right)$ be such that $n_{k}=n_{k-1}+k$ for $k \geq 1$. Then $\mathbf{E}$ is a central $\Lambda(4)$-set for $\mathrm{SU}_{q}(2)$. More precisely, there exists $K_{q} \geq 0$ such that for any finitely supported sequence $\left(c_{n}\right)_{n \in \mathbf{E}} \in \mathbb{C}$,

$$
\left\|\sum_{n \in \mathbf{E}} c_{n} \chi_{n}\right\|_{4} \leq K_{q}\left\|\sum_{n \in \mathbf{E}} c_{n} \chi_{n}\right\|_{2}
$$

Proof. Recall the formulae (1.3) and (1.8). Then for each $m \in \mathbb{N} \cup\{0\}$,

$$
\begin{aligned}
h\left(\chi_{m} \sigma_{-\frac{i}{2}}\left(\chi_{m}\right)\right) & =\sum_{i, j=1}^{m+1} h\left(\left(u_{i i}^{(m)}\right)^{*} \sigma_{-\frac{i}{2}}\left(u_{j j}^{(m)}\right)\right)=\sum_{i, j=1}^{m+1} h\left(\left(u_{i i}^{(m)}\right)^{*}\left(Q_{m}^{1 / 2}\right)_{j j} u_{j j}^{(m)}\left(Q_{m}^{1 / 2}\right)_{j j}\right) \\
& =\sum_{i=1}^{m+1}\left(Q_{m}\right)_{i i} h\left(\left(u_{i i}^{(m)}\right)^{*} u_{i i}^{(m)}\right)=\sum_{i=1}^{m+1}\left(Q_{m}\right)_{i i}\left(Q_{m}^{-1}\right)_{i i} / \operatorname{Tr}\left(Q_{m}\right) \\
& =\frac{m+1}{q^{-m}+q^{-m+2}+\cdots+q^{m-2}+q^{m}} .
\end{aligned}
$$

On the other hand, it is easy to see

$$
\left\|\chi_{m}\right\|_{2}^{2}=h\left(\chi_{m}^{2}\right)=1, \quad\left\|\sum_{n \in \mathbf{E}} c_{n} \chi_{n}\right\|_{2}^{2}=\sum_{n \in \mathbf{E}}\left|c_{n}\right|^{2} .
$$

By (1.2) and (1.3), we also have

$$
h\left(\chi_{m} \sigma_{-\frac{i}{2}} \chi_{n}\right)=0, \quad m \neq n .
$$

Let tr be the trace on the Haagerup $L^{1}$-space $L^{1, \mathrm{H}}\left(\mathrm{SU}_{q}(2)\right)$. Recall Proposition 1.5 - we have for a finitely supported sequence $\left(c_{n}\right)_{n \in \mathbf{E}}$ with $\max _{n}\left|c_{n}\right|=1$ and $f=\sum_{n \in \mathbf{E}} c_{n} \chi_{n}$,

$$
\begin{aligned}
\|f\|_{4}^{4} & =\operatorname{tr}\left(\left|f D^{1 / 4}\right|^{4}\right)=\operatorname{tr}\left(D^{1 / 4} f^{*} f D^{1 / 2} f^{*} f D^{1 / 4}\right) \\
& =\operatorname{tr}\left(f^{*} f\left(D^{1 / 2} f^{*} f D^{-1 / 2}\right) D\right)=h\left(f^{*} f \sigma_{-\frac{i}{2}}\left(f^{*} f\right)\right) \\
& =\sum_{i, j, r, s \in \mathbf{E}} \bar{c}_{i} c_{j} \bar{c}_{r} c_{s} h\left(\chi_{i} \chi_{j} \sigma_{-\frac{i}{2}}\left(\chi_{r} \chi_{s}\right)\right) .
\end{aligned}
$$

Then using (4.18), (4.20) and the fact $\chi_{m} \chi_{m^{\prime}}=\chi_{\left|m-m^{\prime}\right|}+\chi_{\left|m-m^{\prime}\right|+1}+\cdots+\chi_{m+m^{\prime}}$ for any $m, m^{\prime} \in \mathbb{N} \cup\{0\}$, we get

$$
\begin{aligned}
\|f\|_{4}^{4} & \leq \sum_{i, j, r, s \in \mathbf{E}}\left|\bar{c}_{i}\right|\left|c_{j}\right|\left|\bar{c}_{r}\right|\left|c_{s}\right| \sum_{m=\max \{|i-j|,|r-s|\}}^{\min \{i+j, r+s\}}\left|h\left(\chi_{m} \sigma_{-\frac{i}{2}}\left(\chi_{m}\right)\right)\right| \\
& =\sum_{i, j, r, s \in \mathbf{E}}\left|\bar{c}_{i}\right|\left|c_{j}\right|\left|\bar{c}_{r}\right|\left|c_{s}\right| \sum_{m=\max \{|i-j|,|r-s|\}}^{\min \{i+j, r+s\}} \frac{m+1}{q^{-m}+q^{-m+2}+\cdots+q^{m-2}+q^{m}} \\
& \leq \sum_{i, j, r, s \in \mathbf{E}}\left|\bar{c}_{i}\right|\left|c_{j}\right|\left|\bar{c}_{r}\right|\left|c_{s}\right| \sum_{m=\max \{|i-j|,|r-s|\}} \frac{m+1}{q^{-m}},
\end{aligned}
$$


and therefore

$$
\begin{aligned}
\|f\|_{4}^{4} & \leq K \sum_{i, j, r, s \in \mathbf{E}}^{n}\left|\bar{c}_{i}\right|\left|c_{j}\right|\left|\bar{c}_{r}\right|\left|c_{s}\right| \sum_{m=\max \{|i-j|,|r-s|\}}^{\min \{i+j, r+s\}} q^{m / 2} \leq K \sum_{i, j, r, s \in \mathbf{E}}\left|\bar{c}_{i}\left\|c_{j}|| \bar{c}_{r}\right\| c_{s}\right| \frac{q^{\frac{\max \{|i-j|,|r-s|\}}{2}}}{1-q^{1 / 2}} \\
& \leq \frac{K}{1-q^{1 / 2}} \sum_{i, j, r, s \in \mathbf{E}}\left|\bar{c}_{i}\right|\left|c_{j}\right|\left|\bar{c}_{r} \| c_{s}\right| q^{\frac{|i-j|+|r-s|}{4}}=\frac{K}{1-q^{1 / 2}}\left(\sum_{i, j \in \mathbf{E}}\left|\bar{c}_{i}\right|\left|c_{j}\right| q^{\frac{|i-j|}{4}}\right)^{2} \\
& \leq \frac{2 K}{1-q^{1 / 2}}\left(\left(\sum_{i \in \mathbf{E}, i=j}\left|\bar{c}_{i}\right|^{2}\right)^{2}+\left(2 \sum_{i, j \in \mathbf{E}, i<j}\left|c_{i}\right|\left|c_{j}\right| q^{\frac{j-i}{4}}\right)^{2}\right)
\end{aligned}
$$

where $K \geq 1$ is the constant such that $x+1 \leq K q^{-x / 2}$ for $x \geq 1$. Recall the assumption on $\mathbf{E}=\left\{n_{k} \in \mathbb{N} \cup\{0\}: k \geq 0\right\}$ that $n_{k}=n_{k-1}+k$ for $k \geq 1$ and also the assumption $\max _{n}\left|c_{n}\right|=1$, so that $1 \leq\|f\|_{2}$. We have

$$
\begin{aligned}
\sum_{i, j \in \mathbf{E}, i<j}\left|c_{i}\right|\left|c_{j}\right| q^{\frac{j-i}{4}} & =\sum_{k \geq 0}\left|c_{n_{k}}\right| \sum_{l \geq 1}\left|c_{n_{k}+(k+1)+\cdots+(k+l)}\right| q^{\frac{(k+1)+\cdots+(k+l)}{4}} \\
& \leq \sum_{k \geq 0} \sum_{l \geq 1} q^{\frac{(2 k+l+1) l}{8}} \leq \sum_{k \geq 0} \sum_{l \geq 1} q^{\frac{2 k+l+1}{8}} \\
& =\sum_{k \geq 0} q^{\frac{2 k+1}{8}} \sum_{l \geq 1} q^{\frac{l}{8}} \leq q^{1 / 8} \cdot \frac{1}{1-q^{1 / 4}} \cdot \frac{q^{1 / 8}}{1-q^{1 / 8}}:=K^{\prime} .
\end{aligned}
$$

Therefore together with (4.19) we get

$$
\|f\|_{4}^{4} \leq \frac{2 K}{1-q^{1 / 2}}\left(\left(\sum_{i \in \mathbf{E}, i=j}\left|\bar{c}_{i}\right|^{2}\right)^{2}+4 K^{\prime 2}\right) \leq \frac{2 K\left(4 K^{\prime 2}+1\right)}{1-q^{1 / 2}}\|f\|_{2}^{4} .
$$

Take $K_{q}=\left(\frac{2 K\left(4 K^{\prime 2}+1\right)}{1-q^{1 / 2}}\right)^{1 / 4}$ and we get the desired inequality (4.17).

In the end we remark that for the case $0<q<1$, we may also state below the non-existence of infinite $\Lambda(p)$-sets for $\mathrm{SU}_{q}(2)$, which directly follows from Proposition 4.5.

Proposition 4.17. Let $0<q<1$ and $1<p<\infty$. There exist no infinite $\Lambda(p)$-sets or infinite Sidon sets for $\mathrm{SU}_{q}(2)$.

Proof. Recall that the irreducible representations of $\operatorname{Irr}\left(\mathrm{SU}_{q}(2)\right)$ indexed by $\mathbb{N} \cup\{0\}$, and for each $n \in \mathbb{N} \cup\{0\}$ we have $\left\|Q_{n}\right\|=\left\|Q_{n}^{-1}\right\|=q^{-n}$. So for any infinite subset $\mathbf{E} \subset \operatorname{Irr}\left(\mathrm{SU}_{q}(2)\right)$, we have

$$
\sup _{n \in \mathbf{E}}\left\{\left\|Q_{n}\right\|,\left\|Q_{n}^{-1}\right\|\right\}=\infty .
$$

So according to Proposition 4.5, $\mathrm{SU}_{q}(2)$ does not admit any infinite interpolation set for $\mathrm{M}\left(L^{p}(\mathbb{G})\right.$ ), or equivalently, it does not admit any infinite $\Lambda(p)$-set by Theorem 4.7. And by Corollary 4.8, $\mathrm{SU}_{q}(2)$ does not admit any infinite Sidon set.

In the next section we will use another method to show in Corollary 5.9 that a large class of quantum deformations of semi-simple Lie groups do not admit any infinite Sidon set.

\section{Central Sidon sets with examples}

In this final section we briefly discuss some properties of central Sidon sets.

Definition 5.1. (1) We say that a subset $\mathbf{E} \subset \operatorname{Irr}(\mathbb{G})$ is a central Sidon set if there exists $K>0$ such that for any finite sequence $\left(c_{\pi}\right) \subset \mathbb{C}$ and $x=\sum_{\pi} c_{\pi} \chi_{\pi}$, we have $\|\hat{x}\|_{1} \leq K\|x\|_{\infty}$.

(2) A linear functional on $\operatorname{Pol}(\mathbb{G})$ is said to be central if there exists numbers $\left(\omega_{\pi}: \pi \in \operatorname{Irr}(\mathbb{G})\right)$ such that

$$
\hat{\omega}(\pi)=\omega_{\pi} \operatorname{Id}_{\pi}, \quad \pi \in \operatorname{Irr}(\mathbb{G}) .
$$

Denote by $\mathrm{Pol}^{z}(\mathbb{G})=\left\{x=\sum_{\pi \in \operatorname{Irr}(\mathbb{G})} c_{\pi} \chi_{\pi} \in \operatorname{Pol}(\mathbb{G}): c_{\pi} \in \mathbb{C}\right\}$ the subspace of central polynomials and let $C_{r}^{z}(\mathbb{G})$ be the norm closure of $\mathrm{Pol}^{z}(\mathbb{G})$ in $C_{r}(\mathbb{G})$. 
Remark 5.2. Any Sidon set $\mathbf{E} \subset \operatorname{Irr}(\mathbb{G})$ is a central Sidon set for $\mathbb{G}$. In fact, note that for $x=\sum_{\pi} c_{\pi} \chi_{\pi}$ as in the above definition, we have $\hat{x}(\pi)=\operatorname{dim}(\pi)^{-1} c_{\pi} Q_{\pi}^{-1}$ and then by definition,

$$
\|\hat{x}\|_{1}=\sum_{\pi \in \operatorname{Irr}(\mathbb{G})} d_{\pi} \operatorname{Tr}\left(\left|p_{\pi} \hat{x}(\pi) Q_{\pi}\right|\right)=\sum_{\pi \in \operatorname{Irr}(\mathbb{G})} \operatorname{dim}(\pi)\left|c_{\pi}\right| .
$$

Hence $\mathbf{E}$ is a central Sidon set.

Lemma 5.3. Let $\mathbb{G}$ be a compact quantum group. The following assertions are equivalent:

(1) $\mathbb{G}$ is of Kac type;

(2) any central functional $\omega$ is bounded on $\operatorname{Pol}(\mathbb{G})$ (with respect to \|\|$_{\infty}$ ) if and only if it is bounded on $\mathrm{Pol}^{z}(\mathbb{G})$ with the same norm;

$(3)$ there exists a conditional expectation $\mathcal{E}$ from $C_{r}(\mathbb{G})$ onto $C_{r}^{z}(\mathbb{G})$ such that $h \circ \mathcal{E}=h$.

Proof. $(1) \Rightarrow(2)$. Assume that $\mathbb{G}$ is of Kac type. Let $\omega$ be a central functional which is bounded on $\operatorname{Pol}^{z}(\mathbb{G})$. Let $\tilde{\omega} \in L^{\infty}(\mathbb{G})^{*}$ be its Hahn-Banach extension to $L^{\infty}(\mathbb{G})$. Denote by $\mathcal{E}$ the $h$-preserving conditional expectation from $\mathcal{M}=L^{\infty}(\mathbb{G})$ onto the von Neumann subalgebra $\mathcal{N}$ generated by $\operatorname{Pol}^{z}(\mathbb{G})$ in $L^{\infty}(\mathbb{G})$. Recall that for $\pi, \beta \in \operatorname{Irr}(\mathbb{G})$, we have $\chi_{\pi}^{*}=\chi_{\bar{\pi}}$ and $\chi_{\pi} \chi_{\pi^{\prime}}=\chi_{\pi \otimes \pi^{\prime}}$, so the subspace $\operatorname{Pol}^{z}(\mathbb{G})$ spanned by characters is ultraweakly dense in $\mathcal{N}$. Note also that $\mathcal{E}$ is the adjoint map of the embedding $\iota: L^{1}(\mathcal{N}) \rightarrow L^{1}(\mathcal{M})$, so for any $\pi, \pi^{\prime} \in \operatorname{Irr}(\mathbb{G})$ and any $i, j$,

$$
\begin{aligned}
\left\langle\mathcal{E}\left(u_{i j}^{(\pi)}\right), \chi_{\pi^{\prime}}\right\rangle_{L^{1}(\mathcal{N}) *, L^{1}(\mathcal{N})} & =\left\langle u_{i j}^{(\pi)}, \iota\left(\chi_{\pi^{\prime}}\right)\right\rangle_{L^{1}(\mathcal{M})^{*}, L^{1}(\mathcal{M})}=h\left(\left(u_{i j}^{(\pi)}\right)^{*} \chi_{\pi^{\prime}}\right)=\operatorname{dim}(\pi)^{-1} \delta_{i j} \delta_{\pi \pi^{\prime}} \\
& =\operatorname{dim}(\pi)^{-1} \delta_{i j} h\left(\chi_{\pi}^{*} \chi_{\pi^{\prime}}\right)=\left\langle\operatorname{dim}(\pi)^{-1} \delta_{i j} \chi_{\pi}, \chi_{\pi^{\prime}}\right\rangle_{L^{1}(\mathcal{N})^{*}, L^{1}(\mathcal{N})},
\end{aligned}
$$

which means that $E\left(u_{i j}^{(\pi)}\right)=\delta_{i j} \operatorname{dim}(\pi)^{-1} \chi_{\pi}$. Consequently, $\omega=\tilde{\omega} \circ E$ on $\operatorname{Pol}(\mathbb{G})$, and therefore $\omega$ is bounded on $\operatorname{Pol}(\mathbb{G})$ with the same norm.

$(2) \Rightarrow(3)$. Define the linear map $\mathcal{E}: \operatorname{Pol}(\mathbb{G}) \rightarrow \operatorname{Pol}^{z}(\mathbb{G})$ by

$$
\mathcal{E}\left(u_{i j}^{(\pi)}\right)=\delta_{i j} \operatorname{dim}(\pi)^{-1} \chi_{\pi}, \quad \pi \in \operatorname{Irr}(\mathbb{G}) .
$$

It is easy to see that $h \circ \mathcal{E}=h$ on $\operatorname{Pol}(\mathbb{G})$. Also for any central functional $\omega$ on $\operatorname{Pol}(\mathbb{G})$, we have $\omega \circ \mathcal{E}=\omega$. Now for any $x \in \operatorname{Pol}(\mathbb{G})$, by the assertion $(2)$, we have

$$
\|\mathcal{E}(x)\|=\sup _{\omega \in \operatorname{Pol}^{z}(\mathbb{G})^{*},\|\omega\|=1}|\omega(\mathcal{E}(x))|=\sup _{\omega \in \operatorname{Pol}(\mathbb{G})^{*},\|\omega\|=1}|\omega(x)|=\|x\| .
$$

So $\mathcal{E}$ is contractive on $\operatorname{Pol}(\mathbb{G})$ and can be extended to a conditional expectation from $C_{r}(\mathbb{G})$ onto $C_{r}^{z}(\mathbb{G})$ preserving the Haar state.

$(3) \Rightarrow(1)$. Assume $(3)$ holds. It is a standard argument that $\sigma_{t}\left(\mathrm{Pol}^{z}(\mathbb{G})\right) \subset \mathrm{Pol}^{z}(\mathbb{G})$, see e.g. the proof of Theorem 4.2 in [Tak03, Chap.IX]. In fact, let $(H, \Lambda, \pi)$ be the faithful GNS construction of $\operatorname{Pol}(\mathbb{G})$ with respect to the Haar state $h$ and denote by $H_{0}$ the completion of $\Lambda\left(\mathrm{Pol}^{z}(\mathbb{G})\right.$ in $H$. Let $E$ be the orthogonal projection from $H$ onto $H_{0}$. Then for $x \in \operatorname{Pol}(\mathbb{G}), y \in \operatorname{Pol}^{z}(\mathbb{G})$, we have

$$
\langle\Lambda(x), \Lambda(y)\rangle=h\left(x^{*} y\right)=h\left(\mathcal{E}\left(x^{*} y\right)\right)=h\left(\mathcal{E}(x)^{*} y\right)=\langle\Lambda(\mathcal{E}(x)), \Lambda(y)\rangle
$$

so

$$
E(\Lambda(x))=\Lambda(\mathcal{E}(x)), \quad x \in \operatorname{Pol}(\mathbb{G}) .
$$

As usual denote by $\mathscr{S}: \Lambda(x) \mapsto \Lambda\left(x^{*}\right)$ the operator on $H$ induced by involution, and also denote by $\mathfrak{d}$ the modular operator on $\operatorname{Pol}(\mathbb{G})$ associated to the Haar state $h$. Since $\mathcal{E}$ preserves the $*-$ operation, we see that $E \mathscr{S}=\mathscr{S} E$ on the subspace $\Lambda(\operatorname{Pol}(\mathbb{G}))$. Taking adjoint we see that $\mathscr{S}^{*}$ also commutes with $E$ on $\Lambda(\operatorname{Pol}(\mathbb{G}))$, hence so it is for $\mathfrak{d}=\mathscr{S}^{*} \mathscr{S}$. Therefore $\mathfrak{d}^{\mathrm{i} t}$ leaves $\Lambda\left(\operatorname{Pol}^{z}(\mathbb{G})\right.$ invariant for all $t \in \mathbb{R}$, which yields that $\sigma_{t}\left(\mathrm{Pol}^{z}(\mathbb{G})\right) \subset \mathrm{Pol}^{z}(\mathbb{G})$. However we recall that the modular automorphism group acts on $\operatorname{Pol}(\mathbb{G})$ as

$$
\sigma_{t}\left(u_{i j}^{(\pi)}\right)=\sum_{k, l}\left(Q_{\pi}^{\mathrm{i} t}\right)_{i k} u_{k l}^{(\pi)}\left(Q_{\pi}^{\mathrm{i} t}\right)_{l j}, \quad \pi \in \operatorname{Irr}(\mathbb{G}), t \in \mathbb{R}, 1 \leq i, j \leq n_{\pi}
$$

and hence

$$
\sigma_{t}\left(\chi_{\pi}\right)=\sigma_{t}\left(\sum_{i} u_{i i}^{(\pi)}\right)=\sum_{k, l}\left(\sum_{i}\left(Q_{\pi}^{\mathrm{i} t}\right)_{l i}\left(Q_{\pi}^{\mathrm{i} t}\right)_{i k}\right) u_{k l}^{(\pi)}=\sum_{k, l}\left(Q_{\pi}^{2 \mathrm{i} t}\right)_{l k} u_{k l}^{(\pi)}, \quad \pi \in \operatorname{Irr}(\mathbb{G}) t \in \mathbb{R} .
$$


So the invariance $\sigma_{t}\left(\mathrm{Pol}^{z}(\mathbb{G})\right) \subset \mathrm{Pol}^{z}(\mathbb{G})$ yields that $Q_{\pi}=\operatorname{Id}_{\pi}$ for all $\pi \in \operatorname{Irr}(\mathbb{G})$, that is, $\mathbb{G}$ is of Kac type.

Proposition 5.4. Let $\mathbb{G}$ be a compact quantum group of Kac type. Then $\mathbf{E} \subset \operatorname{Irr}(\mathbb{G})$ is a central Sidon set if and only if for all bounded sequences $\left(a_{\pi}\right)_{\pi \in \mathbf{E}} \subset \mathbb{C}$, there exists a bounded central functional $\varphi \in C_{r}(\mathbb{G})^{*}$ such that $\hat{\varphi}(\pi)=a_{\pi} \operatorname{Id}_{\pi}$ for $\pi \in \mathbf{E}$.

Proof. The proof is an analogue of that of Theorem $3.3(1) \Leftrightarrow(2)$. We replace $C_{r}(\mathbb{G})$ by $C_{r}^{z}(\mathbb{G})$ and $\ell^{\infty}(\mathbf{E})$ by $\left(\ell^{\infty}(\mathbf{E})\right)^{z}=\left\{\left(a_{\pi}\right) \in \ell^{\infty}(\mathbf{E}): a_{\pi} \in \mathbb{C I d}_{\pi}, \pi \in \operatorname{Irr}(\mathbb{G})\right\}$, etc., and argue as before, thanks to the previous lemma.

Remark 5.5. There have been some suggestions on the definition of unconditional Sidon sets for a compact (quantum) group which would be different from that in Definition 3.13. More precisely, for a compact quantum group $\mathbb{G}$ and a subset $\mathbf{E} \subset \operatorname{Irr}(\mathbb{G})$, we may consider the following lacunary condition: there exists a constant $K>0$ such that

$$
\forall\left(\varepsilon_{\pi}\right)_{\pi \in \mathbf{E}} \subset\{-1,1\}, \quad\left\|\sum_{\pi \in \mathbf{E}} d_{\pi} \varepsilon_{\pi}(\iota \otimes \operatorname{Tr})\left(\left(1 \otimes \hat{x}(\pi) Q_{\pi}\right) u^{(\pi)}\right)\right\|_{\infty} \leq K\|x\|_{\infty}, \quad x \in \operatorname{Pol}_{\mathbf{E}}(\mathbb{G}) .
$$

And it was ever unclear on the relations between (5.2) and the Sidon sets even if $\mathbb{G}$ is a compact group $G$. Here we remark that the above two notions of lacunarity are in fact totally different if $G$ is non-abelian. Indeed, more generally, if the compact quantum group $\mathbb{G}$ is coamenable and of Kac type, we may follow the same idea as in Theorem 3.15 and the above proposition to see that (5.2) holds if and only if $\mathbf{E} \subset \operatorname{Irr}(\mathbb{G})$ is a central Sidon set for $\mathbb{G}$, which is well-known to be different from being a Sidon set.

Remark 5.6. For a central functional $\omega$ on $\operatorname{Pol}(\mathbb{G})$, the associated multiplier map $T_{\omega}=(\omega \otimes$ id $) \circ \Delta$ acts as $u_{i j}^{(\pi)} \mapsto \omega_{\pi} u_{i j}^{(\pi)}$ on $\operatorname{Pol}(\mathbb{G})$. In [DCFY14, Sect.2] it is proved that if $\Phi: \operatorname{Irr}\left(\mathbb{G}_{1}\right) \rightarrow \operatorname{Irr}\left(\mathbb{G}_{2}\right)$ is a monoidal equivalence between two compact quantum groups $\mathbb{G}_{1}$ and $\mathbb{G}_{2}$ and if $\omega^{(1)}, \omega^{(2)}$ are the central functionals on $\operatorname{Pol}\left(\mathbb{G}_{1}\right), \operatorname{Pol}\left(\mathbb{G}_{2}\right)$ respectively, such that $\omega_{\pi}^{(1)}=\omega_{\Phi(\pi)}^{(2)}$, then $T_{\omega(1)}$ and $T_{\omega^{(2)}}$ have the same complete bounded norms. Note that if additionally $\mathbb{G}_{1}$ and $\mathbb{G}_{2}$ are coamenable, then $\left\|\omega^{(1)}\right\|=\left\|T_{\omega^{(1)}}\right\|=\left\|T_{\omega^{(2)}}\right\|=\left\|\omega^{(2)}\right\|$ since $\omega=\omega \star \epsilon=(\mathrm{id} \otimes \epsilon) \circ T_{\omega}$ for any functional $\omega$ on $\operatorname{Pol}(\mathbb{G})$. Then according to the previous proposition, any two coamenable compact quantum groups of Kac type which are monoidally equivalent, have a one-to-one correspondence of their central Sidon sets via the monoidal equivalence map. The following result generalizes this fact.

Proposition 5.7. Let $\mathbb{G}_{1}, \mathbb{G}_{2}$ be two compact quantum groups. Assume that $\Phi: \operatorname{Rep}\left(\mathbb{G}_{1}\right) \rightarrow$ $\operatorname{Rep}\left(\mathbb{G}_{2}\right)$ is an injective map preserving the fusion rules, that is, for all $\pi, \pi^{\prime} \in \operatorname{Rep}\left(\mathbb{G}_{1}\right)$ we have

$$
\Phi\left(\pi \otimes \pi^{\prime}\right)=\Phi(\pi) \otimes \Phi\left(\pi^{\prime}\right), \quad \Phi\left(\oplus_{i=1}^{n} \pi_{i}\right)=\oplus_{i=1}^{n} \Phi\left(\pi_{i}\right), \quad \pi, \pi^{\prime}, \pi_{i} \in \operatorname{Rep}(G), n \geq 1 .
$$

Then for any finite sequence $\left(c_{\pi}\right) \subset \mathbb{C}$ and $x=\sum_{\pi \in \operatorname{Irr}\left(\mathbb{G}_{1}\right)} c_{\pi} \chi_{\pi} \in \operatorname{Pol}\left(\mathbb{G}_{1}\right)$, we have

$$
\left\|\sum_{\pi \in \operatorname{Irr}\left(\mathbb{G}_{1}\right)} c_{\pi} \chi_{\pi}\right\|_{L^{\infty}\left(\mathbb{G}_{1}\right)}=\left\|\sum_{\pi \in \operatorname{Irr}\left(\mathbb{G}_{1}\right)} c_{\pi} \chi_{\Phi(\pi)}\right\|_{L^{\infty}\left(\mathbb{G}_{2}\right)} .
$$

Consequently, for any central Sidon set $\mathbf{E} \subset \operatorname{Irr}\left(\mathbb{G}_{1}\right)$, if additionally there exists $C>0$ satisfying $\operatorname{dim}(\Phi(\pi)) \leq C \operatorname{dim}(\pi)$ for all $\pi \in \mathbf{E}$, then $\Phi(\mathbf{E}) \subset \operatorname{Irr}\left(\mathbb{G}_{2}\right)$ is a central Sidon set for $\mathbb{G}_{2}$.

Proof. The isometry (5.3) has been mentioned in [Ban99]. In fact, as remarked in [Ban99], the map $\Phi$ automatically satisfies $\Phi(\bar{\pi})=\overline{\Phi(\pi)}$ for all $\pi \in \operatorname{Rep}(\mathbb{G})$ once we note that for an irreducible representation $\pi$ its adjoint $\bar{\pi}$ is the unique irreducible representation such that 1 is the subrepresentation of $\pi \otimes \bar{\pi}$. Denote by $A$ the $*$-algebra generated by $\left\{\chi_{\pi}: \pi \in \operatorname{Irr}\left(\mathbb{G}_{1}\right)\right\}$. By the linear independence of $\left\{\chi_{\pi}: \pi \in \operatorname{Irr}\left(\mathbb{G}_{1}\right)\right\}$, the injection $\Phi$ induces an injective $*$-homomorphism

$$
\tilde{\Phi}: A \rightarrow \operatorname{Pol}\left(\mathbb{G}_{2}\right), \quad \chi_{\pi} \mapsto \chi_{\Phi(\pi)}, \pi \in \operatorname{Rep}\left(\mathbb{G}_{1}\right) .
$$

To see that $\tilde{\Phi}$ well defines a $*$-homomorphism, it suffices to notice that for $\pi \in \operatorname{Irr}\left(\mathbb{G}_{1}\right)$, we have $\chi_{\pi}^{*}=\chi_{\bar{\pi}}$ and

$$
\tilde{\Phi}\left(\chi_{\pi}^{*}\right)=\tilde{\Phi}\left(\chi_{\bar{\pi}}\right)=\chi_{\Phi(\bar{\pi})}=\chi_{\overline{\Phi(\pi)}}=\tilde{\Phi}\left(\chi_{\pi}\right)^{*}
$$


and for $\pi, \pi^{\prime} \in \operatorname{Rep}\left(\mathbb{G}_{1}\right)$ satisfying the decomposition formula $\pi \otimes \pi^{\prime}=\sum_{k} \pi_{k}$ with each $\pi_{k} \in$ $\operatorname{Irr}\left(\mathbb{G}_{1}\right)$, we have

$$
\tilde{\Phi}\left(\chi_{\pi} \chi_{\pi^{\prime}}\right)=\sum_{k} \tilde{\Phi}\left(\chi_{\gamma_{k}}\right)=\sum_{k} \chi_{\Phi\left(\gamma_{k}\right)}=\chi_{\Phi(\pi)} \chi_{\Phi\left(\pi^{\prime}\right)}=\tilde{\Phi}\left(\chi_{\pi}\right) \tilde{\Phi}\left(\chi_{\pi^{\prime}}\right) .
$$

Note that $\tilde{\Phi}$ preserves the restriction of Haar states on $\operatorname{Pol}^{z}\left(\mathbb{G}_{1}\right)$ and $\operatorname{Pol}^{z}\left(\mathbb{G}_{2}\right)$. So $\tilde{\Phi}$ gives rise to an equivalence between the faithful sub-GNS-representations of $\operatorname{Pol}^{z}\left(\mathbb{G}_{1}\right) \subset \operatorname{Pol}\left(\mathbb{G}_{1}\right)$ and $\operatorname{Pol}^{z}\left(\mathbb{G}_{2}\right) \subset \operatorname{Pol}\left(\mathbb{G}_{2}\right)$ with respect to the Haar states. As a result $\tilde{\Phi}$ is an isometry, which gives (5.3). The assertion regarding the central Sidon sets then follows directly from the definition and (5.1).

Example 5.8. Let $q \in[-1,1] \backslash\{0\}$ and consider the quantum groups $\mathrm{SU}_{q}(n), n \geq 2 . \mathrm{SU}_{q}(n)$ is a compact matrix quantum group in the sense of [Wor87] and we denote by $\pi_{n}^{(q)}$ the fundamental representation of $\mathrm{SU}_{q}(n)$. It is easy to see that for the classical case $q=1$, the subset $\left\{\pi_{n}^{(1)}: n \geq 2\right\}$ is a Sidon set for the compact group $\prod_{n \geq 2} \mathrm{SU}(n)$ (see [CM81, p.308-310]). Now by Proposition 1.3 for $0<q<1$ we may find a map $\Phi: \operatorname{Irr}\left(\mathrm{SU}_{q}(n)\right) \rightarrow \operatorname{Irr}(\mathrm{SU}(n))$ satisfying the assumptions of the above proposition. As a result, for any $0<q<1$ the subset $\left\{\pi_{n}^{(q)}: n \geq 2\right\}$ is a central Sidon set for the compact quantum group $\prod_{n \geq 2} \mathrm{SU}_{q}(n)$.

In [Rid72] it is shown that for a connected compact group $G, G$ has an infinite central Sidon set if and only if $G$ is not a semi-simple Lie group. Combined with Proposition 1.3 and Proposition 5.7 we get the following observation, which in particular shows that the quantum $\mathrm{SU}_{q}(2)(0<q<1)$ does not admit any infinite Sidon set, as mentioned in the last section after Proposition 4.16.

Corollary 5.9. For all $0<q<1$ and all simply connected compact semi-simple Lie group $G$, the compact quantum group $G_{q}$ given by the Drinfeld-Jimbo deformation does not admit any infinite central Sidon set.

\section{ApPEndix: Existence of $\Lambda(p)$-SETS IN ORTHOGONAL SYSTEMS FOR GENERAL NONCOMMUTATIVE $L^{p}$-SPACES}

In this appendix we present a method of constructing $\Lambda(p)$-sets in orthogonal systems for noncommutative $L^{p}$-spaces. The main result is the following theorem. It is due to Marek Bożejko in the tracial case.

Theorem A.1. Let $\mathcal{M}$ be a von Neumann algebra equipped with a normal faithful state $\varphi$ and consider the associated $L^{p}$-spaces $L^{p}(\mathcal{M}, \varphi)$. Let $B=\left\{x_{i} \in \mathcal{M}: i \geq 1\right\}$ be an orthogonal system with respect to $\varphi$ (i.e., $\varphi\left(x_{i}^{*} x_{j}\right)=0$ for all $i \neq j$ ) such that $\sup _{i}\left\|x_{i}\right\|_{\infty}<\infty$. Then for each $2<p<\infty$, there exists an infinite subset $\left\{x_{i_{k}}: k \geq 1\right\} \subset B$ and a constant $C>0$ such that for all finitely supported sequences $\left(c_{k}\right) \subset \mathbb{C}$ we have

$$
\left\|\sum_{k \geq 1} c_{k} x_{i_{k}}\right\|_{p} \leq C\left(\sum_{k \geq 1}\left|c_{k}\right|^{2}\right)^{\frac{1}{2}} .
$$

This result was first proved by [KS36] in the commutative case and then by [Boż79] in the case where $\varphi$ is tracial. The same idea also applies to the general setting, and for the sake of completeness we include a detailed proof below. We refer to [Pic73, Boż73, Boż75b] for different approaches.

To establish the theorem, let us show the following slightly stronger result.

Theorem A.2. Let $\mathcal{M}$ and $\varphi$ be given as in Theorem $A .1$ and $n \geq 2$. Let $B=\left\{x_{i} \in L^{2 n}(\mathcal{M}, \varphi)\right.$ : $i \geq 1\}$ be an orthogonal system with respect to $\varphi$ such that $\sup _{i}\left\|x_{i}\right\|_{2 n}<\infty$. Then there exists an infinite subset $\left\{x_{i_{k}}: k \geq 1\right\} \subset B$ and a constant $C>0$ such that for all finitely supported sequences $\left(c_{k}\right) \subset \mathbb{C}$ we have

$$
\left\|\sum_{k \geq 1} c_{k} x_{i_{k}}\right\|_{2 n} \leq C\left(\sum_{k \geq 1}\left|c_{k}\right|^{2}\right)^{\frac{1}{2}}
$$


Proof. Write $K=\max \left\{1, \sup _{i}\left\|x_{i}\right\|_{2 n}\right\}<\infty$. Denote by $\mathcal{M}_{a}$ the subalgebra of all analytic elements in $\mathcal{M}$. Recall that $\mathcal{M}_{a}$ a ultraweakly dense subspace of $\mathcal{M}$, and for all $x \in \mathcal{M}_{a}$, the analytic extension $\sigma_{z}(x) \in \mathcal{M}$ for $z \in \mathbb{C}$ is well-defined (see [Tak03]). In particular $\mathcal{M}_{a}$ is also a dense subspace of $L^{p}(\mathcal{M}, \varphi)$ for $1 \leq p<\infty$ according to Lemma 1.8.

(1) Firstly, assume that $B \subset \mathcal{M}_{a}$. Note that for any $y \in \mathcal{M}_{a}$,

$$
\varphi\left(y x_{k}\right) \rightarrow 0, \quad \varphi\left(x_{k} y\right) \rightarrow 0, \quad k \rightarrow \infty .
$$

We choose a subset $\left\{x_{i_{k}}: k \geq 1\right\} \subset B$ inductively as follows. Let $x_{i_{1}}=x_{1}$ and if $\left\{x_{i_{j}}: 1 \leq j \leq k\right\}$ for $k \geq 1$ is chosen, we take an $x_{i_{k+1}} \in B$ such that for all $1 \leq k_{0}, k_{1}, \ldots, k_{n-1}, l_{1}, \ldots, l_{n-1} \leq k$,

$$
\left|\varphi\left(\sigma_{\frac{(n-1) \mathrm{i}}{n}}\left(x_{i_{k_{1}}}^{*} x_{i_{l_{1}}}\right) \sigma_{\frac{(n-2) \mathrm{i}}{n}}\left(x_{i_{k_{2}}}^{*} x_{i_{l_{2}}}\right) \cdots \sigma_{\frac{\mathrm{i}}{n}}\left(x_{i_{k_{n-1}}}^{*} x_{i_{l_{n-1}}}\right) x_{i_{k_{0}}}^{*} x_{i_{k+1}}\right)\right| \leq \frac{1}{2 k^{2 n-1}(k+1)}
$$

and

$$
\left|\varphi\left(\sigma_{\mathrm{i}}\left(x_{i_{k_{0}}}\right) \sigma_{\frac{(n-1) \mathrm{i}}{n}}\left(x_{i_{k_{1}}}^{*} x_{i_{l_{1}}}\right) \sigma_{\frac{(n-2) \mathrm{i}}{n}}\left(x_{i_{k_{2}}}^{*} x_{i_{l_{2}}}\right) \cdots \sigma_{\frac{\dot{i}}{n}}\left(x_{i_{k_{n-1}}^{*}}^{*} x_{i_{l_{n-1}}}\right) x_{i_{k+1}}^{*}\right)\right| \leq \frac{1}{2 k^{2 n-1}(k+1)} .
$$

This can always be done by the orthogonality of $B$. In fact, since the elements in $B$ are assumed to be analytic, we have

$$
\begin{gathered}
\sigma_{\frac{(n-1) \mathrm{i}}{n}}\left(x_{i_{k_{1}}}^{*} x_{i_{l_{1}}}\right) \sigma_{\frac{(n-2) \mathrm{i}}{n}}\left(x_{i_{k_{2}}}^{*} x_{i_{l_{2}}}\right) \cdots \sigma_{\frac{\dot{i}}{n}}\left(x_{i_{k_{n-1}}}^{*} x_{i_{l_{n-1}}}\right) x_{i_{k_{0}}}^{*} \in \mathcal{M}\left(\subset L^{2}(\mathcal{M}, \varphi)\right), \\
\sigma_{\mathrm{i}}\left(x_{i_{k_{0}}}\right) \sigma_{\frac{(n-1) \mathrm{i}}{n}}\left(x_{i_{k_{1}}}^{*} x_{i_{l_{1}}}\right) \sigma_{\frac{(n-2) \mathrm{i}}{n}}\left(x_{i_{k_{2}}}^{*} x_{i_{l_{2}}}\right) \cdots \sigma_{\frac{\mathrm{i}}{n}}\left(x_{i_{k_{n-1}}}^{*} x_{i_{l_{n-1}}}\right) \in \mathcal{M}\left(\subset L^{2}(\mathcal{M}, \varphi)\right) .
\end{gathered}
$$

Note that there exist only finitely many elements of the above forms for each given $k \geq 1$. So the element $x_{i_{k+1}}$ can be well chosen. With the same notation we deduce the following inequalities,

$$
\begin{aligned}
& \left|\operatorname{tr}\left(D^{\frac{1}{2 n}} x_{i_{k_{1}}}^{*} x_{i_{l_{1}}} D^{\frac{1}{2 n}} D^{\frac{1}{2 n}} x_{i_{k_{2}}}^{*} x_{i_{l_{2}}} D^{\frac{1}{2 n}} \cdots D^{\frac{1}{2 n}} x_{i_{k_{n-1}}}^{*} x_{i_{l_{n-1}}} D^{\frac{1}{2 n}} D^{\frac{1}{2 n}} x_{i_{k_{0}}}^{*} x_{i_{k+1}} D^{\frac{1}{2 n}}\right)\right| \\
= & \left|\operatorname{tr}\left(D \sigma_{\frac{(n-1) \mathrm{i}}{n}}\left(x_{i_{k_{1}}}^{*} x_{i_{l_{1}}}\right) \sigma_{\frac{(n-2) \mathrm{i}}{n}}\left(x_{i_{k_{2}}}^{*} x_{i_{l_{2}}}\right) \cdots \sigma_{\frac{\mathrm{i}}{n}}\left(x_{i_{k_{n-1}}}^{*} x_{i_{l_{n-1}}}\right) x_{i_{k_{0}}}^{*} x_{i_{k+1}}\right)\right| \\
= & \left|\varphi\left(\sigma_{\frac{(n-1) \mathrm{i}}{n}}\left(x_{i_{k_{1}}}^{*} x_{i_{l_{1}}}\right) \sigma_{\frac{(n-2) \mathrm{i}}{n}}\left(x_{i_{k_{2}}}^{*} x_{i_{l_{2}}}\right) \cdots \sigma_{\frac{\mathrm{i}}{n}}\left(x_{i_{k_{n-1}}}^{*} x_{i_{l_{n-1}}}\right) x_{i_{k_{0}}}^{*} x_{i_{k+1}}\right)\right| \leq \frac{1}{2 k^{2 n-1}(k+1)},
\end{aligned}
$$

and similarly

$$
\left|\operatorname{tr}\left(D^{\frac{1}{2 n}} x_{i_{k_{1}}}^{*} x_{i_{l_{1}}} D^{\frac{1}{2 n}} \cdots D^{\frac{1}{2 n}} x_{i_{k_{n-1}}}^{*} x_{i_{l_{n-1}}} D^{\frac{1}{2 n}} D^{\frac{1}{2 n}} x_{i_{k+1}}^{*} x_{i_{k_{0}}} D^{\frac{1}{2 n}}\right)\right| \leq \frac{1}{2 k^{2 n-1}(k+1)} .
$$

Fix a sequence $\left(c_{k}\right) \subset \mathbb{C}$ such that $\sum_{k \geq 1}\left|c_{k}\right|^{2}=1$. Take $r \geq 1$ and write

$$
g_{r}=\sum_{k=1}^{r} c_{k} x_{i_{k}} .
$$

Since then each $\left|c_{k}\right| \leq 1$ for $k \geq 1$, the above inequalities yield that

$$
\left|\operatorname{tr}\left(\left(D^{\frac{1}{2 n}} g_{r}^{*} g_{r} D^{\frac{1}{2 n}}\right)^{n-1} D^{\frac{1}{2 n}} g_{r}^{*} x_{i_{r+1}} D^{\frac{1}{2 n}}\right)\right| \leq r^{2 n-1} \cdot \frac{1}{2(r+1) r^{2 n-1}}=\frac{1}{2(r+1)},
$$

and similarly

$$
\left|\operatorname{tr}\left(\left(D^{\frac{1}{2 n}} g_{r}^{*} g_{r} D^{\frac{1}{2 n}}\right)^{n-1} D^{\frac{1}{2 n}} x_{i_{r+1}}^{*} g_{r} D^{\frac{1}{2 n}}\right)\right| \leq \frac{1}{2(r+1)} .
$$

Now for each $r \geq 1$ write $I_{r}=\left\|\sum_{k=1}^{r} c_{k} x_{i_{k}}\right\|_{2 n}^{2 n}$. Then

$$
I_{r+1}=\left\|\sum_{k=1}^{r+1} c_{k} x_{i_{k}}\right\|_{2 n}^{2 n}=\left\|g_{r}+c_{r+1} x_{i_{r+1}}\right\|_{2 n}^{2 n}=\operatorname{tr}\left(\left(D^{\frac{1}{2 n}}\left(g_{r}+c_{r+1} x_{i_{r+1}}\right)^{*}\left(g_{r}+c_{r+1} x_{i_{r+1}}\right) D^{\frac{1}{2 n}}\right)^{n}\right) .
$$

We may write

$$
I_{r+1}=J_{1}+J_{2}+J_{3}+J_{4},
$$

where

$$
J_{1}=\operatorname{tr}\left(\left(D^{\frac{1}{2 n}} g_{r}^{*} g_{r} D^{\frac{1}{2 n}}\right)^{n}\right)=I_{r}, \quad J_{2}=\operatorname{tr}\left(\left(D^{\frac{1}{2 n}}\left(c_{r+1} x_{i_{r+1}}\right)^{*}\left(c_{r+1} x_{i_{r+1}}\right) D^{\frac{1}{2 n}}\right)^{n}\right),
$$


with

$$
\begin{gathered}
J_{3}=\sum_{\underline{y} \in A_{1}} \operatorname{tr}\left(D^{\frac{1}{2 n}} y_{1}^{*} y_{1}^{\prime} D^{\frac{1}{2 n}} D^{\frac{1}{2 n}} y_{2}^{*} y_{2}^{\prime} D^{\frac{1}{2 n}} \cdots D^{\frac{1}{2 n}} y_{n}^{*} y_{n}^{\prime} D^{\frac{1}{2 n}}\right), \\
J_{4}=\sum_{j=2}^{n-1} \sum_{\underline{y} \in A_{j}} \operatorname{tr}\left(D^{\frac{1}{2 n}} y_{1}^{*} y_{1}^{\prime} D^{\frac{1}{2 n}} D^{\frac{1}{2 n}} y_{2}^{*} y_{2}^{\prime} D^{\frac{1}{2 n}} \cdots D^{\frac{1}{2 n}} y_{n}^{*} y_{n}^{\prime} D^{\frac{1}{2 n}}\right),
\end{gathered}
$$

$$
\begin{aligned}
A_{j}=\{ & \underline{y}=\left(y_{1}, \ldots, y_{n}, y_{1}^{\prime}, \ldots, y_{n}^{\prime}\right): y_{k}, y_{k}^{\prime} \in\left\{g_{r}, c_{r+1} x_{i_{r+1}}\right\}, 1 \leq k \leq n, \\
& \left.\operatorname{Card}\left\{k: y_{k}=x_{i_{r+1}}\right\}+\operatorname{Card}\left\{k: y_{k}^{\prime}=x_{i_{r+1}}\right\}=j\right\} .
\end{aligned}
$$

We see that

$$
\left|J_{2}\right|=\left|c_{r+1}\right|^{2}\left\|x_{i_{r+1}}\right\|_{2 n}^{2 n} \leq K^{2 n}\left|c_{r+1}\right|^{2} .
$$

By traciality of tr and by (A.2)-(A.3) we have

$$
\begin{aligned}
\left|J_{3}\right|= & \mid n c_{r+1} \operatorname{tr}\left(\left(D^{\frac{1}{2 n}} g_{r}^{*} g_{r} D^{\frac{1}{2 n}}\right)^{n-1} D^{\frac{1}{2 n}} g_{r}^{*} x_{i_{r+1}} D^{\frac{1}{2 n}}\right) \\
& \quad+n c_{r+1} \operatorname{tr}\left(\left(D^{\frac{1}{2 n}} g_{r}^{*} g_{r} D^{\frac{1}{2 n}}\right)^{n-1} D^{\frac{1}{2 n}} x_{i_{r+1}}^{*} g_{r} D^{\frac{1}{2 n}}\right) \mid \\
\leq & \frac{n\left|c_{r+1}\right|}{r+1} .
\end{aligned}
$$

By the Hölder inequality

SO

$$
\left|\operatorname{tr}\left(D^{\frac{1}{2 n}} y_{1}^{*} y_{1}^{\prime} D^{\frac{1}{2 n}} D^{\frac{1}{2 n}} y_{2}^{*} y_{2}^{\prime} D^{\frac{1}{2 n}} \cdots D^{\frac{1}{2 n}} y_{n}^{*} y_{n}^{\prime} D^{\frac{1}{2 n}}\right)\right| \leq \prod_{k=1}^{n}\left\|y_{k}\right\|_{2 n}\left\|y_{k}^{\prime}\right\|_{2 n}
$$

$$
\left|J_{4}\right| \leq \sum_{j=2}^{n} 2^{2 n}\left\|g_{r}\right\|_{2 n}^{n-j}\left\|c_{r+1} x_{i_{r+1}}\right\|_{2 n}^{j} \leq K^{n} 4^{n} n\left|c_{r+1}\right|^{2}\left(I_{r}+1\right) .
$$

Combining (A.4)-(A.7) we have

$$
I_{r+1} \leq\left(1+K^{n} 4^{n} n\left|c_{r+1}\right|^{2}\right) I_{r}+\left(K^{2 n}+K^{n} 4^{n} n\right)\left|c_{r+1}\right|^{2}+\frac{n\left|c_{r+1}\right|}{r+1} .
$$

Iterating the above inequality and denoting $a_{k}=K^{n} 4^{n} n\left|c_{k}\right|^{2}, b_{k}=\left(K^{2 n}+K^{n} 4^{n} n\right)\left|c_{k}\right|^{2}+\frac{n\left|c_{k}\right|}{k}$ for each $k \geq 1$, we obtain for $r \geq 2$

$$
\begin{aligned}
I_{r} & \leq \prod_{k=2}^{r}\left(1+a_{k}\right) I_{1}+\sum_{l=2}^{r-1} b_{l} \prod_{j=l+1}^{r}\left(1+a_{j}\right)+b_{r} \\
& \leq e^{\sum_{k \geq 1} a_{k}} I_{1}+e^{\sum_{k \geq 1} a_{k}} \sum_{l \geq 1} b_{l} \leq\left(2 K^{2 n}+K^{n} 4^{n} n+n \sum_{l \geq 1} l^{-2}\right) e^{K^{n} 4^{n} n} .
\end{aligned}
$$

The above right hand side is a constant only depending on $K$ and $n$, so the desired inequality (A.1) is proved.

(2) Now choose an arbitrary family $B=\left\{x_{i} \in L^{2 n}(\mathcal{M}, \varphi): i \geq 1\right\}$ satisfying the assumption. Write $p=2 n$. Without loss of generality we take $K \geq 1$ in the sequel. By the density of $\mathcal{M}_{a}$ in $L^{p}(\mathcal{M}, \varphi)$, we choose the sequences $\left(x_{k}^{\prime}\right),\left(y_{k}\right) \subset \mathcal{M}_{a}$ and $\left(j_{k}\right)_{k \geq 1} \subset \mathbb{N}$ inductively as follows. Let $j_{1}=1$ and $x_{1}^{\prime}=y_{1} \in \mathcal{M}_{a}$ such that

$$
\left\|x_{1}-x_{1}^{\prime}\right\|_{p} \leq 2^{-1}
$$

If $x_{l}^{\prime}, y_{l}, j_{l}$ for $1 \leq l \leq k$ are chosen, take by orthogonality a $j_{k+1} \geq j_{k}$ such that for all $1 \leq l \leq k$,

$$
\left|\varphi\left(y_{l}^{*} x_{j_{k+1}}\right)\right| \leq 2^{-k-l-2}\left\|y_{l}\right\|_{2}^{2}\left\|y_{l}\right\|_{p}^{-1}
$$

and by the density property choose $x_{k+1}^{\prime} \in \mathcal{M}_{a}$ such that

$$
\left\|x_{j_{k+1}}-x_{k+1}^{\prime}\right\|_{p} \leq 2^{-k-2}\left(1+\sum_{l=1}^{k} \frac{\left\|y_{l}\right\|_{p}}{\left\|y_{l}\right\|_{2}}\right)^{-1}
$$


where we always make the standard convention in this proof that the terms such as $\left\|y_{l}\right\|_{2}^{2} /\left\|y_{l}\right\|_{p}^{-1}$ and $\left\|y_{l}\right\|_{p} /\left\|y_{l}\right\|_{2}^{-1}$ are regarded as zero if $y_{l}=0$. Also set

$$
y_{k+1}=x_{k+1}^{\prime}-\sum_{l=1}^{k} \varphi\left(y_{l}^{*} y_{l}\right)^{-1} \varphi\left(y_{l}^{*} x_{k+1}^{\prime}\right) y_{l} .
$$

We see immediately that $\left(y_{k}\right)$ is an orthogonal system. Moreover, when we recall the orthogonality of $\left(x_{k}\right)$, by the choice of the above sequences we have

$$
\begin{aligned}
\left\|x_{j_{k+1}}-y_{k+1}\right\|_{p} & \leq\left\|x_{j_{k+1}}-x_{k+1}^{\prime}\right\|_{p}+\sum_{l=1}^{k} \frac{\left\|y_{l}\right\|_{p}}{\left\|y_{l}\right\|_{2}^{2}}\left|\varphi\left(y_{l}^{*} x_{k+1}^{\prime}\right)\right| \\
& =\left\|x_{j_{k+1}}-x_{k+1}^{\prime}\right\|_{p}+\sum_{l=1}^{k} \frac{\left\|y_{l}\right\|_{p}}{\left\|y_{l}\right\|_{2}^{2}}\left|\varphi\left(y_{l}^{*}\left(x_{k+1}^{\prime}-x_{j_{k+1}}\right)\right)+\varphi\left(y_{l}^{*} x_{j_{k+1}}\right)\right| \\
& \leq\left\|x_{j_{k+1}}-x_{k+1}^{\prime}\right\|_{p}+\sum_{l=1}^{k} \frac{\left\|y_{l}\right\|_{p}}{\left\|y_{l}\right\|_{2}^{2}}\left\|y_{l}\right\|_{2}\left\|x_{j_{k+1}}-x_{k+1}^{\prime}\right\|_{2}+\sum_{l=1}^{k} 2^{-k-l-2} \\
& \leq\left\|x_{j_{k+1}}-x_{k+1}^{\prime}\right\|_{p}\left(1+\sum_{l=1}^{k} \frac{\left\|y_{l}\right\|_{p}}{\left\|y_{l}\right\|_{2}}\right)+2^{-k-2} \leq 2^{-k-1} .
\end{aligned}
$$

By the argument in (1), the orthogonal system $\left\{y_{k}: k \geq 1\right\} \subset \mathcal{M}_{a}$ admits a subset $\left\{y_{k_{r}}: r \geq 1\right\}$ satisfying the estimation of the type (A.1). Now it is easy to observe that $\left\{x_{j_{k_{r}}}: r \geq 1\right\} \subset B$ is the desired subset. To see this, it suffices to note that, there exists a constant $C>0$ such that for all sequences $\left(c_{r}\right)_{r \geq 1} \subset \mathbb{C}$ with $\sum_{r}\left|c_{r}\right|^{2}=1$ and for all $s \geq 1$,

$$
\left\|\sum_{r=1}^{s} c_{r} y_{k_{r}}\right\|_{p} \leq C
$$

and hence by the estimates (A.8),

$$
\left\|\sum_{r=1}^{s} c_{r} x_{j_{k_{r}}}\right\|_{p} \leq\left\|\sum_{r=1}^{s} c_{r} y_{k_{r}}\right\|_{p}+\sum_{r=1}^{s}\left|c_{r}\right|\left\|x_{j_{k_{r}}}-y_{k_{r}}\right\|_{p} \leq C+\sum_{r=1}^{s}\left|c_{r}\right| 2^{-r} \leq C+1 .
$$

So the proof is complete.

Remark. In general, the noncommutative $L^{p}$-spaces introduced by [Kos84] depend on different interpolation parameters. Following the same line of the proof one can see that the above result still holds for those types of $L^{p}$-spaces.

Acknowledgment. The author is indebted to his advisors Quanhua Xu and Adam Skalski for their helpful discussions and constant encouragement. He would also like to thank Professors Marek Bożejko and Marius Junge for fruitful communications and suggestions, and thank Professor Gilles Pisier for his careful reading and pointing out a mistake in the preprint version. The research was partially supported by the ANR Project ANR-11-BS01-0008 and the NCN (National Centre of Science) grant 2014/14/E/ST1/00525.

\section{REFERENCES}

[Ban99] T. Banica. Fusion rules for representations of compact quantum groups. Exposition. Math., 17(4):313$337,1999$.

[BL76] J. Bergh and J. Löfström. Interpolation spaces. An introduction. Springer-Verlag, Berlin-New York, 1976. Grundlehren der Mathematischen Wissenschaften, No. 223.

[BM13] T. Blendek and J. Michaliček. $L^{1}$-norm estimates of character sums defined by a Sidon set in the dual of a compact Kac algebra. J. Operator Theory, 70(2):375-399, 2013.

[Boż73] M. Bożejko. The existence of $\Lambda(p)$ sets in discrete noncommutative groups. Boll. Un. Mat. Ital. (4), 8:579-582, 1973.

[Boż74] M. Bożejko. Sidon sets in dual objects of compact groups. Colloq. Math., 30:137-141, 1974.

[Boż75a] M. Bożejko. On $\Lambda(p)$ sets with minimal constant in discrete noncommutative groups. Proc. Amer. Math. Soc., 51:407-412, 1975. 
[Boż75b] M. Bożejko. A remark to my paper: "The existence of $\Lambda(p)$ sets in discrete noncommutative groups" (Boll. Un. Mat. Ital. (4) 8 (1973), 579-582). Boll. Un. Mat. Ital. (4), 11(1):43, 1975.

[Boż79] M. Bożejko. Lacunary sets in finite von neumann algebras. Unpublished manuscript, 1979.

[Boż81] M. Bożejko. A new group algebra and lacunary sets in discrete noncommutative groups. Studia Math., 70(2):165-175, 1981.

[Bra12] M. Brannan. Quantum symmetries and strong Haagerup inequalities. Comm. Math. Phys., 311(1):21-53, 2012.

[BT03] E. Bédos and L. Tuset. Amenability and co-amenability for locally compact quantum groups. Internat. J. Math., 14(8):865-884, 2003.

[Cas13] M. Caspers. The $L^{p}$-Fourier transform on locally compact quantum groups. J. Operator Theory, 69(1):161-193, 2013.

[CM81] D. I. Cartwright and J. R. McMullen. A structural criterion for the existence of infinite Sidon sets. Pacific J. Math., 96(2):301-317, 1981.

[Coo10] T. Cooney. A Hausdorff-Young inequality for locally compact quantum groups. Internat. J. Math., 21(12):1619-1632, 2010

[Daw10] M. Daws. Multipliers, self-induced and dual Banach algebras. Dissertationes Math. (Rozprawy Mat.), 470:62, 2010

[Daw12] M. Daws. Completely positive multipliers of quantum groups. Internat. J. Math., 23(12):1250132, 23, 2012.

[DCFY14] K. De Commer, A. Freslon, and M. Yamashita. CCAP for universal discrete quantum groups. Comm. Math. Phys., 331(2):677-701, 2014, With an appendix by Stefaan Vaes.

[Dru70] S. W. Drury. Sur les ensembles de Sidon. C. R. Acad. Sci. Paris Sér. A-B, 271:A162-A163, 1970.

[ER00] E. G. Effros and Z.-J. Ruan. Operator spaces, volume 23 of London Mathematical Society Monographs. New Series. The Clarendon Press, Oxford University Press, New York, 2000.

[Eym64] P. Eymard. L'algèbre de Fourier d'un groupe localement compact. Bull. Soc. Math. France, 92:181-236, 1964.

[Fol95] G. B. Folland. A course in abstract harmonic analysis. Studies in Advanced Mathematics. CRC Press, Boca Raton, FL, 1995.

[FT77] A. Figà-Talamanca. Lacunary sets in noncommutative groups. Rend. Sem. Mat. Fis. Milano, 47:45-59 (1979), 1977

[FTR66] A. Figà-Talamanca and D. Rider. A theorem of Littlewood and lacunary series for compact groups. Pacific J. Math., 16:505-514, 1966.

[Har99] A. Harcharras. Fourier analysis, Schur multipliers on $S^{p}$ and non-commutative $\Lambda(p)$-sets. Studia Math., 137(3):203-260, 1999.

[HJX10] U. Haagerup, M. Junge, and Q. Xu. A reduction method for noncommutative $L_{p}$-spaces and applications. Trans. Amer. Math. Soc., 362(4):2125-2165, 2010.

[HR70] E. Hewitt and K. A. Ross. Abstract harmonic analysis. Vol. II: Structure and analysis for compact groups. Analysis on locally compact Abelian groups. Die Grundlehren der mathematischen Wissenschaften, Band 152. Springer-Verlag, New York-Berlin, 1970.

[JNR09] M. Junge, M. Neufang, and Z.-J. Ruan. A representation theorem for locally compact quantum groups. Internat. J. Math., 20(3):377-400, 2009.

[Jun02] M. Junge. Doob's inequality for non-commutative martingales. J. Reine Angew. Math., 549:149-190, 2002.

[Jun05] M. Junge. Embedding of the operator space $O H$ and the logarithmic 'little Grothendieck inequality'. Invent. Math., 161(2):225-286, 2005.

[JX03] M. Junge and Q. Xu. Noncommutative Burkholder/Rosenthal inequalities. Ann. Probab., 31(2):948-995, 2003.

[Kah10] B.-J. Kahng. Fourier transform on locally compact quantum groups. J. Operator Theory, 64(1):69-87, 2010.

[Kos84] H. Kosaki. Applications of the complex interpolation method to a von Neumann algebra: noncommutative $L^{p}$-spaces. J. Funct. Anal., 56(1):29-78, 1984.

[KS36] S. Kaczmarz and H. Steinhaus. Theorie der Orthogonalreihen. Monografie Matematyczne. Instytut Matematyczny Polskiej Akademi Nauk, Warszawa-Lwów, 1936

[KS97] A. Klimyk and K. Schmüdgen. Quantum groups and their representations. Texts and Monographs in Physics. Springer-Verlag, Berlin, 1997.

[Lei74] M. Leinert. Faltungsoperatoren auf gewissen diskreten Gruppen. Studia Math., 52:149-158, 1974.

[LS91] S. Levendorskiı and Y. Soibelman. Algebras of functions on compact quantum groups, Schubert cells and quantum tori. Comm. Math. Phys., 139(1):141-170, 1991

[Lun09] A. Lunardi. Interpolation theory. Appunti. Scuola Normale Superiore di Pisa (Nuova Serie). [Lecture Notes. Scuola Normale Superiore di Pisa (New Series)]. Edizioni della Normale, Pisa, second edition, 2009.

[Meg98] R. E. Megginson. An introduction to Banach space theory, volume 183 of Graduate Texts in Mathematics. Springer-Verlag, New York, 1998. 
[MP81] M. B. Marcus and G. Pisier. Random Fourier series with applications to harmonic analysis, volume 101 of Annals of Mathematics Studies. Princeton University Press, Princeton, N.J.; University of Tokyo Press, Tokyo, 1981.

[MVD98] A. Maes and A. Van Daele. Notes on compact quantum groups. Nieuw Arch. Wisk. (4), 16(1-2):73-112, 1998.

[NT13] S. Neshveyev and L. Tuset. Compact quantum groups and their representation categories, volume 20 of Cours Spécialisés [Specialized Courses]. Société Mathématique de France, Paris, 2013.

[Pic73] M. A. Picardello. Lacunary sets in discrete noncommutative groups. Boll. Un. Mat. Ital. (4), 8:494-508, 1973.

[Pis78a] G. Pisier. Ensembles de Sidon et espaces de cotype 2. In Séminaire sur la Géométrie des Espaces de Banach (1977-1978), pages Exp. No. 14, 12. Ecole Polytech., Palaiseau, 1978.

[Pis78b] G. Pisier. Sur l'espace de Banach des séries de Fourier aléatoires presque sûrement continues. In Séminaire sur la Géométrie des Espaces de Banach (1977-1978), pages Exp. No. 17-18, 33. École Polytech., Palaiseau, 1978.

[Pis83a] G. Pisier. Conditions d'entropie et caractérisations arithmétiques des ensembles de Sidon. In Topics in modern harmonic analysis, Vol. I, II (Turin/Milan, 1982), pages 911-944. Ist. Naz. Alta Mat. Francesco Severi, Rome, 1983.

[Pis83b] G. Pisier. Some applications of the metric entropy condition to harmonic analysis. In Banach spaces, harmonic analysis, and probability theory (Storrs, Conn., 1980/1981), volume 995 of Lecture Notes in Math., pages 123-154. Springer, Berlin, 1983.

[Pis95] G. Pisier. Multipliers and lacunary sets in non-amenable groups. Amer. J. Math., 117(2):337-376, 1995.

[PX03] G. Pisier and Q. Xu. Non-commutative $L^{p}$-spaces. In Handbook of the geometry of Banach spaces, Vol. 2, pages 1459-1517. North-Holland, Amsterdam, 2003.

[Rid72] D. Rider. Central lacunary sets. Monatsh. Math., 76:328-338, 1972.

[Rid75] D. Rider. Randomly continuous functions and Sidon sets. Duke Math. J., 42(4):759-764, 1975.

[Rud60] W. Rudin. Trigonometric series with gaps. J. Math. Mech., 9:203-227, 1960.

[RX06] É. Ricard and Q. Xu. Khintchine type inequalities for reduced free products and applications. J. Reine Angew. Math., 599:27-59, 2006.

[Soi90] Y. Soibelman. Algebra of functions on a compact quantum group and its representations. Algebra $i$ Analiz, 2(1):190-212, 1990.

[Tak02] M. Takesaki. Theory of operator algebras. I, volume 124 of Encyclopaedia of Mathematical Sciences. Springer-Verlag, Berlin, 2002. Reprint of the first (1979) edition, Operator Algebras and Noncommutative Geometry, 5.

[Tak03] M. Takesaki. Theory of operator algebras. II, volume 125 of Encyclopaedia of Mathematical Sciences. Springer-Verlag, Berlin, 2003. Operator Algebras and Non-commutative Geometry, 6.

[Ter81] M. Terp. $L_{p}$ spaces associated with von neumann algebras. In Notes, Report No. $3 a+3 b$. Københavns Universitets Matematiske Institut, 1981.

[Tim08] T. Timmermann. An invitation to quantum groups and duality. EMS Textbooks in Mathematics. European Mathematical Society (EMS), Zürich, 2008. From Hopf algebras to multiplicative unitaries and beyond.

[VD96] A. Van Daele. Discrete quantum groups. J. Algebra, 180(2):431-444, 1996.

[VD07] A. Van Daele. The Fourier transform in quantum group theory. In New techniques in Hopf algebras and graded ring theory, pages 187-196. K. Vlaam. Acad. Belgie Wet. Kunsten (KVAB), Brussels, 2007.

[VDW96] A. Van Daele and Shuzhou Wang. Universal quantum groups. Internat. J. Math., 7(2):255-263, 1996.

[Ver07] R. Vergnioux. The property of rapid decay for discrete quantum groups. J. Operator Theory, 57(2):303324, 2007.

[Voi98] D. Voiculescu. A strengthened asymptotic freeness result for random matrices with applications to free entropy. Internat. Math. Res. Notices, (1):41-63, 1998.

[Wan95a] Shuzhou Wang. Free products of compact quantum groups. Comm. Math. Phys., 167(3):671-692, 1995.

[Wan95b] Shuzhou Wang. Tensor products and crossed products of compact quantum groups. Proc. London Math. Soc. (3), 71(3):695-720, 1995

[Wan16] Simeng Wang. $L_{p}$-improving convolution operators on finite quantum groups. Indiana Univ. Math. J., 65:1609-1637, 2016.

[Wor87] S. L. Woronowicz. Compact matrix pseudogroups. Comm. Math. Phys., 111(4):613-665, 1987.

[Wor98] S. L. Woronowicz. Compact quantum groups. In Symétries quantiques (Les Houches, 1995), pages 845884. North-Holland, Amsterdam, 1998.

Laboratoire de Mathématiques, Université de Franche-Comté, 25030 Besançon Cedex, France and Institute of Mathematics, Polish Academy of Sciences, ul. Śniadeckich 8, 00-956 Warszawa, Poland

E-mail address: simeng.wang@univ-fcomte.fr 